\title{
RECURSIVE FUNCTIONS AND INTUITIONISTIC NUMBER THEORY
}

\author{
BY \\ DAVID NELSON
}

The purpose of this paper is to examine, for propositions of elementary number theory, the relation between an intuitionistic truth definition and the provability of formulas which represent these propositions in formal systems of intuitionistic logic. The intuitionistic truth definition is that of Kleene( $\left.{ }^{1}\right)$. He has arithmetized this truth notion in the definition of a syntactical predicate "the natural number $a$ realizes the number-theoretic formula $A$ " $[15, \S 5]$. A familiarity wi . this definition as well as other portions of Kleene's paper will be presupposed for the following.

The present results relate the constructibility demands of the intuitionists as embodied in a formal system of number theory by Heyting to Church's thesis identifying constructible and general recursive functions $[8 ; 9 ; 3]$.

In Part I, it is shown that certain systems of intuitionistic number theory satisfy the definition for realizability. We demonstrate that any formula deducible from a realizable formula in one of these formal systems is realizable. This result enables us, by the reasoning of Kleene $[15, \S 10]$, to show that certain classically true formulas are not provable in intuitionistic number theory. Likewise, it affords a demonstration that certain classically true formulas of the predicate calculus are unprovable in the intuitionistic predicate calculus.

Then, following Kleene's notation [15, §§11-12], we demonstrate that the formulas $A \supset \AA A$ and $® A \supset A$ are realizable. This result concerns an interpretative consistency proof for a system of intuitionistic number theory which is an extension of the usual formalization $[15, \S 13]$.

Parts II and III take up the problem of formalizing a portion of the theory of recursive functions and predicates in intuitionistic number theories. Part II is concerned principally with the formalization of material which is familiar on the intuitive plane and much of which has already been formalized in the framework of a classical logic. We utilize the work of Hilbert and Bernays [10, vol. 1] and Gödel [6] to avoid additional laborious detail. Part III introduces formulas $\mathrm{R}_{k}\left(\mathrm{e}, \mathrm{a}_{1}, \cdots, \mathrm{a}_{k}, \mathrm{~b}\right)$ representing primitive recursive predicates having properties similar to Kleene's predicates $T_{k}\left(e, a_{1}, \cdots, a_{k}, b\right)$ $[13, \S \S 4,7]$, but defined in a way which facilitates formalization of the theory of general and partial recursive functions and predicates. These formulas en-

Presented to the Society, September 17, 1945; received by the editors May 13, 1946.

(1) [15]. Numbers in brackets refer to the bibliography at the end of the paper. 
able us to develop an abbreviation for the representation of partial and general recursive functions in a particular system $S_{3}$.

Part IV contains a formalization of the conclusions of Part I, which, as Kleene has observed $[15, \S 14]$, furnishes a metamathematical consistency proof for a broader intuitionistic logic containing formulas, which, upon their usual interpretation, contradict certain classically true number-theoretic propositions. Formal results are also obtained which afford a metamathematical refinement of the proofs, both of the incompleteness of the unextended formalization of intuitionistic number theory, and of the unprovability of certain formulas in the intuitionistic predicate calculus $[15, \S \S 15,16]$.

\section{PART I}

1. Formal systems of intuitionistic number theory. We shall employ the formal symbolism as it is described in the introductory article by Kleene [ 15 , \$], except that letters from the early part of the alphabet will be reserved for free variables and letters from the latter part for bound variables. For the present theory, this amounts to an inessential difference. Unless an identification is explicitly stated, it is assumed, when reference is made to the variables of a formula, that distinct letters stand for distinct variables.

In the first system $S_{1}$ the only function and predicate symbols which appear are' (successor), + (sum), $\cdot$ (product), and = (equality). Other function and predicate symbols as described in the reference may appear in the additional systems which we wish to consider.

The deductive postulates for $S_{1}$ will be the following axiom schemata and rules of inference, from which the notions of formal proof and deducibility from assumption formulas are defined in the familiar way.

We note that the system $S_{1}$ differs from a classical system of number theory $C_{1}$ only in Axiom Schema 8, which in the classical system has the form 77A $\supset$ A [1, pp. 44, 48].

In the postulate list, $A, B, C$ represent formulas, $a, b, c$ represent free variables, $t$ represents a term, $A(b)$ represents a formula containing the free. variable $b$, and $x$ represents a bound variable not occurring in $A(b)$, and $A(x)$, $A(t)$, and so on, represent the results of substituting $x, t$, and so on, for $b$ throughout the formula $A(b)$. Further restriction is stated accompanying the two rules of inference in which it is required.

Throughout the remainder of the work, if, for a given formula $A(a)$ and for any bound variable $x$, we write $\operatorname{VxA}(x)$ or $\operatorname{GxA}(\mathrm{x})$, it will be understood that $x$ is distinct from all the bound variables of $A(a)$.

Axiom schemata and rules of inference for the predicate calculus $\left({ }^{2}\right)$

1a. $A \supset(B \supset A)$.

(2) The postulates 1-12 give a system for the predicate calculus equivalent to Gentzen's $\mathrm{I}-\mathrm{J}$ calculus [4]. Cf. also [8]. 
1b. $(A \supset(B \supset C)) \supset((A \supset B) \supset(A \supset C))$.

2. $\frac{\mathrm{A}, \mathrm{A} \supset \mathrm{B}}{\mathrm{B}}$.

3. $A \supset(B \supset A \& B)$.

4a. $A \& B \supset A$.

4b. A \& B つ B.

5a. $A \supset A \vee B$.

5b. $B \supset A \vee B$.

6. $(A \supset C) \supset((B \supset C) \supset(A \vee B \supset C))$.

7. $(\mathrm{A} \supset \mathrm{B}) \supset((\mathrm{A} \supset>\mathrm{B}) \supset>\mathrm{A})$.

8. $7 \mathrm{~A} \supset(\mathrm{A} \supset \mathrm{B})$.

9. $\frac{C \supset A(b)}{C \supset V \times A(x)}$.

In Rule 9, the variable b must not occur in C.

10. $\operatorname{VxA}(\mathrm{x}) \supset \mathrm{A}(\mathrm{t})$.

11. $\mathrm{A}(\mathrm{t}) \supset \mathbb{H} \mathrm{XA}(\mathrm{x})$.

12. $\frac{\mathrm{A}(\mathrm{b}) \supset \mathrm{C}}{\mathrm{G} \mathrm{xA}(\mathrm{x}) \supset \mathrm{C}}$.

In Rule 12, the variable b must not occur in $C$.

$$
\text { Axioms for }=\text { and Peano axioms }
$$

13. $A(0) \& \forall x\left(A(x) \supset A\left(x^{\prime}\right)\right) \supset A(b)$.

14. $a^{\prime}=b^{\prime} \supset a=b$.

15. $7 a^{\prime}=0$.

16. $a=a$.

17. $a=b \supset(a=c \supset b=c)$.

18. $a=b \supset a^{\prime}=b^{\prime}$.

Axioms for the function symbols + and .

19. $a+0=a$.

20. $a+b^{\prime}=(a+) b^{\prime}$.

21. $a \cdot 0=0$.

22. $a \cdot b^{\prime}=(a \cdot b)+a$.

The schema for $=$, 


\section{3. $\mathrm{b}=\mathrm{c} \supset(\mathrm{A}(\mathrm{b}) \supset \mathrm{A}(\mathrm{c}))$,}

may appear in other systems of intuitionistic number theory.

The formal systems of the types listed by Kleene $[15, \S 4, \mathrm{~A}-\mathrm{E}]$, and also of types intermediate in this classification, may, in addition to Postulates 118, contain Axiom Schema 23 and axioms for further function and predicate symbols which represent general recursive functions and predicates. We summarize the relation between terms and elementary formulas of the formal systems and intuitive functions and predicates in a lemma.

LEMMA 1. Under the assigned interpretation of the formal function and predicate symbols and the usual interpretation of composition, each term $t\left(a_{1}, \cdots, a_{k}\right)$ and each elementary formula $\mathrm{A}\left(\mathrm{a}_{1}, \cdots, \mathrm{a}_{k}\right)$, both containing exactly the free variables displayed, will represent, respectively, a general recursive function $t\left(x_{1}, \cdots, x_{k}\right)$ and a general recursive predicate $A\left(x_{1}, \cdots, x_{k}\right)$.

We shall require a familiarity with the following recursive functions for the discussion of Part I. All the functions except (k), (l), and (m) are primitive recursive $[5 ; 11 ; 13]$, and these three are partial recursive functions $[12 ; 13]$.

\section{LIST OF RECURSIVE FUNCTIONS}

(a) $U_{m}^{n}\left(x_{1}, \cdots, x_{n}\right)$. The identity functions, the $m$ th variable in the list of $n$ variables which are arguments of the function [7].

(b) $x^{\prime}$. The successor function, $x+1$.

(c) $0(x), 1(x), \cdots$ The constant functions, $0,1, \cdots$

(d) $x+y$. The sum of $x$ and $y$.

(e) $x \cdot y$. The product of $x$ and $y$.

(f) $x^{y}$. The $y$ power of $x$.

(g) $(x)_{n}$. If $x=0$, the value of the function is the exponent of the $(n+1)$ st prime in a factorization of $x$ into a product of powers of distinct primes (including primes to the power 0$)\left({ }^{3}\right)$, for example, if $x=2^{1} \cdot 3^{0} \cdot 5^{9} \cdot 7^{4}$, then $(x)_{2}=9$. The value of $(0)_{n}$ is 0 .

(h) $\beta(x)$. The function which takes the value 0 when $x \neq 0$ and takes the value 1 when $x=0[10$, vol. 1, p. 311].

(i) $\epsilon(x, y)$. The function $\left({ }^{4}\right)$ which takes the value 0 when $x=y$ and takes the value 1 when $x \neq y$.

(j) $S_{n}^{m}\left(z, y_{1}, \cdots, y_{m}\right)$. If $\phi\left(y_{1}, \cdots, y_{m}, x_{1}, \cdots, x_{n}\right)$ is a function of the $m+n$ variables $y_{1}, \cdots, y_{m}, x_{1}, \cdots, x_{n}$ in that order and is defined recursively by $e$, and $k_{1}, \cdots, k_{m}$ are natural numbers, then $S_{n}^{m}\left(e, k_{1}, \cdots, k_{m}\right)$ defines recursively $\phi\left(k_{1}, \cdots, k_{m}, x_{1}, \cdots, x_{n}\right)$ as a function of the remaining variables $x_{1}, \cdots, x_{n}[12, \S 2]$.

( $\left.{ }^{3}\right)$ The notation was first used in $[13, \S 5]$, but note the difference in the definition of the function from $[14,85]$; our $(x)_{n}$ is $(x)_{n+1}$ as defined there.

( 4 ) $[10$, vol. 1 , pp. $302 \mathrm{ff}$.]. The present $\epsilon(x, y)$ corresponds to the function $\alpha(\delta(x, y)$ $+\delta(y, x))$, defined there. 
(Since the application of the function $S_{n}^{m}\left(z, y_{1}, \cdots, y_{m}\right)$ requires that $\phi\left(y_{1}, \cdots, y_{m}, x_{1}, \cdots, x_{n}\right)$ be considered as a function of the variables in that specific order, to avoid ambiguity, we introduce the notation " $\lambda x_{1} \cdots x_{n} t$ " for an expression " $t$ " taken as a function of the variables $x_{1} \cdots, x_{n}$ in that order; this notation also serves to distinguish the variables from parameters which may appear ${ }^{(5)}$.)

(k) $\operatorname{\mu yR} R\left(x_{1}, \cdots, x_{n}, y\right)$. For every partial recursive relation $R\left(x_{1}, \cdots, x_{n}\right.$, $y), \mu y R\left(x_{1}, \cdots, x_{n}, y\right)$ defines a partial recursive function which specifies the least $y$ such that $R\left(x_{1}, \cdots, x_{n}, y\right)$; the range of definition of the function is all $x_{1}, \cdots, x_{n}$ such that $(E y) R\left(x_{1}, \cdots, x_{n}, y\right)[13, \S 3]$.

(l) $\Phi_{n}\left(z, x_{1}, \cdots, x_{n}\right)$. If the recursive function $\phi\left(x_{1}, \cdots, x_{n}\right)$ is defined by the Gödel number $e$, then $\phi\left(x_{1}, \cdots, x_{n}\right)=\Phi_{n}\left(e, x_{1}, \cdots, x_{n}\right)$. The function $\Phi_{n}\left(z, x_{1}, \cdots, x_{n}\right)$ is a partially defined function, the range of definition is all $z, x_{1}, \cdots, x_{n}$ such that $(E y) T_{n}\left(z, x_{1}, \cdots, x_{n}, y\right)[12, \S 2]$.

(m) $\Phi_{1}^{n}\left(z, x_{1}, \cdots, x_{n}\right)$. We introduce this function generated by iterations of $\Phi_{1}(z, x)$ for notational brevity, since it is frequently used below. It is defined by the equation

$$
\Phi_{1}^{n}\left(z, x_{1}, \cdots, x_{n}\right)=\Phi_{1}\left(\cdots \Phi_{1}\left(z, x_{1}\right), \cdots, x_{n}\right) .
$$

(n) $S_{k}(e)$. This function has the value $u_{1}$, where $u_{1}$ is the Gödel number which defines the function $\lambda x_{1} S_{1}^{1}\left(u_{2}, x_{1}\right)$, where $u_{2}$, in turn, defines the function $\lambda x_{1} x_{2} S_{1}^{2}\left(u_{3}, x_{1}, x_{2}\right), \cdots$, where $u_{k-1}$ defines the function $\lambda x_{1} \cdots x_{k-1} S_{1}^{k-1}\left(u_{k}, x_{1}\right.$, $\left.\cdots, x_{k-1}\right)$, where $u_{k}=e$.

(It should be noted that $\lambda x_{1} \cdots x_{j} \Phi_{1}^{j}\left(S_{k}(e), x_{1}, \cdots, x_{j}\right)$, for $j<k$, is the same function as $\lambda x_{1} \cdots x_{j} S_{1}^{j}\left(u_{j+1}, x_{1}, \cdots, x_{j}\right)$. Also, $\lambda x_{1} \cdots x_{k} \Phi_{1}^{k}\left(S_{k}(e), x_{1}\right.$, $\left.\cdots, x_{k}\right)$ is the same function as $\lambda x_{1} \cdots x_{k} \Phi_{k}\left(e, x_{1}, \cdots, x_{k}\right)$.)

(o) $E_{k}\left(x_{1}, \cdots, x_{k}\right)$. This function for $k \geqq 2$ is introduced merely for notational brevity. $E_{2}\left(x_{1}, x_{2}\right)=2^{x_{1}} \cdot 3^{x_{2}} ;$ and $E_{k+1}\left(x_{1}, x_{2}, \cdots, x_{k+1}\right)=2^{x_{1}}$ $\cdot 3^{E_{k}\left(x_{2}, \cdots, x_{k+1}\right)}$.

Henceforth we shall assume a familiarity with the concept of realizability as defined by Kleene $[15, \S 5]$. We proceed to the statement of several lemmas which are fundamental to Theorem 1 .

For the statement and proof of Lemma 2, we establish the following notational conventions. Let $\mathrm{A}(\mathrm{a})$ be a formula containing no free variables except a. Let $t\left(a_{1}, \cdots, a_{k}\right)$ be a term containing exactly the free variables $\mathrm{a}_{1}, \cdots, \mathrm{a}_{k}$ which represents a general recursivefunction $\lambda x_{1} \cdots x_{k} t\left(x_{1}, \cdots, x_{k}\right)$, cf. Lemma 1 , and let $n=t\left(n_{1}, \cdots, n_{k}\right)$. Under these conventions, we have the following.

Lemma 2. The formula $\mathrm{A}\left(\mathrm{t}\left(\boldsymbol{n}_{1}, \cdots, \boldsymbol{n}_{k}\right)\right)$ is realized by $m$ if and only if the formula $\mathrm{A}(\mathrm{n})$ is realized by $m$.

(8) The notation is taken from [2], but with a slight change in use. (Church's $\lambda$ was defined for functions of one variable, and functions of $n$ variables were obtained by iteration.) 
The lemma is proved by induction. The basis follows immediately from Lemma 1 . Since $n=t\left(n_{1}, \cdots, n_{k}\right)$, a necessary and sufficient condition for $A(n)$ is $A\left(t\left(n_{1}, \cdots, n_{k}\right)\right)$. And if one of the formulas is realized, then the other is also, and if either is realized, it is realized by 0 .

For the induction step, there are six cases, one for each of the logical symbols. We shall note the one for the conjunction symbol and the universal operator; the others proceed in a similar fashion. (1) For the conjunction symbol there are three subcases: (i) Both A and B contain a. If $\mathrm{A}(\boldsymbol{n}) \& \mathrm{~B}(\boldsymbol{n})$ is realized by $m$, then $\mathrm{A}(\boldsymbol{n})$ is realized by $(m)_{0}$, and $\mathrm{B}(\boldsymbol{n})$ by $(m)_{1}$; then $\mathrm{A}\left(\mathrm{t}\left(\boldsymbol{n}_{1}, \cdots, \boldsymbol{n}_{k}\right)\right)$ and $\mathrm{B}\left(\mathrm{t}\left(\boldsymbol{n}_{1}, \cdots, \boldsymbol{n}_{k}\right)\right)$, by the induction hypothesis, are realized by $(m)_{0}$ and $(m)_{1}$, respectively, and $\mathrm{A}\left(\mathrm{t}\left(\boldsymbol{n}_{1}, \cdots, \boldsymbol{n}_{k}\right)\right) \& \mathrm{~B}\left(\mathrm{t}\left(\boldsymbol{n}_{1}, \cdots, \boldsymbol{n}_{k}\right)\right)$ is realized by $2^{(m)_{0} \cdot 3^{(m) 1}}$ or $m$. Reversing these steps, we obtain the converse. (ii) and (iii) Suppose that either the first or the second of the two formulas of the conjunction does not contain $n$, the treatment proceeds again as in (i).

(2) If $\forall x A(x, n)$ is realized by $k$, then $k$ defines a recursive function $\lambda x \phi(x)$, which, for any numeral $n_{0}$, gives a realization for $\mathrm{A}\left(\boldsymbol{n}_{0}, \boldsymbol{n}\right)$. By the induction hypothesis, $\mathrm{A}\left(\boldsymbol{n}_{0}, \mathrm{t}\left(\boldsymbol{n}_{1}, \cdots, \boldsymbol{n}_{k}\right)\right)$ is also realized by $\phi\left(n_{0}\right)$, which allows us to take the Gödel number of $\lambda x \phi(x)$ as a realization for $\forall \times A\left(x, t\left(n_{1}, \cdots, n_{k}\right)\right)$.

For the statement of Lemmas 3 and 4 we shall first explain some abbreviations in notation. If $a_{k_{1}}, \cdots, a_{\kappa_{l}}$ comprise all the free variables occurring in a formula $B$ and they occur in the formula in the order in which they are listed, and if $n_{k_{1}}, \cdots, n_{k_{l}}$ are any natural numbers, then $\mathrm{B}^{*}$ is the expression obtained by replacing $a_{k_{1}}, \cdots, a_{k_{l}}$, respectively, by $n_{k_{1}}, \cdots, n_{k_{l}}$ throughout B.

Let the natural numbers $1, \cdots, n$ be partitioned into two increasing sequences, $M$ which is $\mu_{1}, \therefore, \mu_{j}$ and $N$ which is $\nu_{1}, \cdots, \nu_{k}$. Suppose that $\mathrm{A}_{p_{1}}, \cdots, \mathrm{A}_{\boldsymbol{p}_{k}}, \mathrm{~A}$ are formulas which contain collectively the free variables $a_{\mu_{1}}, \cdots, a_{\mu_{j}}$ and contain no other free variables and contain none of the bound variables $\mathrm{x}_{\mu_{1}}, \cdots, \mathrm{x}_{\mu_{j}}$, and suppose in addition that, for any $i$ which is in $N$, the formula $\mathrm{A}_{i}$ contains only those of the free variables $\mathrm{a}_{h}$ for which $h<i$.

Then we define a set of operations $\Omega_{i}$ for $1 \leqq i \leqq n$ :

(1) If $i$ is in $N$ and $\mathrm{B}$ is a formula, then $\Omega_{i} \mathrm{~B}$ is the formula $\mathrm{A}_{i} \supset \mathrm{B}$.

(2) If $i$ is in $M$ and $\mathrm{B}\left(\mathrm{a}_{i}\right)$ is a formula containing the free variable $\mathrm{a}_{i}$ and not containing the bound variable $\mathrm{x}_{i}$, then $\Omega_{i} \mathrm{~B}\left(\mathrm{a}_{i}\right)$ is the formula $\forall \mathrm{x}_{i} \mathrm{~B}\left(\mathrm{x}_{i}\right)$.

For example, suppose $B(a)$ and $C(a, b)$ contain exactly the free variables indicated. If we make the following identifications: $x_{1}$ with $y, A_{2}\left(a_{1}\right)$ with $B(a), x_{3}$ with $z$, and $A\left(a_{1}, a_{3}\right)$ with $C(a, b)$, then $\Omega_{1} \Omega_{2} \Omega_{3} A\left(a_{1}, a_{3}\right)$ will be the same formula as $\mathrm{Vy}(\mathrm{B}(\mathrm{y}) \supset \mathrm{VzC}(\mathrm{y}, \mathrm{z}))$.

Let us assume in addition that, according as $i$ is in $M$ or in $N$, " $\zeta_{i}$ " abbreviates " $n_{i}$ " or " $a_{i}$," where $n_{i}$ is any natural number and $a_{i}$ is any number which realizes $\mathrm{A}_{i}^{*}$. 
When we say "for every $\zeta_{1}, \cdots, \zeta_{n}$ " we mean for every choice of $n_{i}$ and every choice of $a_{h}$ which realizes $\mathrm{A}_{h}{ }^{*}$, made in the specific order $\zeta_{1}, \cdots, \zeta_{n}$. Note that the choice of $a_{h}$ will, in general, depend on the choice of the numerals appearing for the free variables of $A_{h}$, that is, on the choice of the $n_{i}$, where $i<h$.

Lemma 3. If $c$ realizes $\Omega_{1} \cdots \Omega_{n} \mathrm{~A}$, then, for every choice of $\zeta_{1}, \cdots, \zeta_{n}$, the formula $\mathrm{A}^{*}$ is realized by $\Phi_{1}^{n}\left(c, \zeta_{1}, \cdots, \zeta_{n}\right)$.

We make two preliminary observations required for the proof. If $B(a)$ is a formula containing no free variables except a and not containing the bound variable $\mathrm{x}$, and if $e$ realizes $\mathrm{VxB}(\mathrm{x})$, then $\mathrm{B}(n)$ is realized by $\Phi_{1}(e, n)$.

If $\mathrm{C}$ and $\mathrm{D}$ are formulas containing no free variables, and if $e$ realizes $\mathrm{C} \supset \mathrm{D}$, and $c$ realizes $\mathrm{C}$, then $\mathrm{D}$ is realized by $\Phi_{1}(e, c)$.

The proof then follows by induction. If the formula $\left(\Omega_{p} \cdots \Omega_{n} A\right)^{*}$ is realized by $\Phi_{1}^{p-1}\left(c, \zeta_{1}, \cdots, \zeta_{p-1}\right)$, then in either case for $p$ the formula $\left(\Omega_{p+1} \cdots \Omega_{n} \mathrm{~A}\right) *$ is realized by the number $\Phi_{1}\left(\Phi_{1}^{p-1}\left(c, \zeta_{1}, \cdots, \zeta_{p-1}\right), \zeta_{p}\right)$, but this is the same number as $\Phi_{1}^{p}\left(c, \zeta_{1}, \cdots, \zeta_{p}\right)$ (cf. Definition $\mathrm{m}$ ).

LEMMA 4. If, for every choice of $\zeta_{1}, \cdots, \zeta_{n}$, the number $\Phi_{n}\left(e, \zeta_{1}, \cdots, \zeta_{n}\right)$ realizes $\mathrm{A}^{*}$, then $\Omega_{1} \cdots \Omega_{n} \mathrm{~A}$ is realized by $S_{n}(e)$.

Again an induction proof gives the desired result. The number $S_{n}(e)$ will realize $\Omega_{1} \ldots \Omega_{n} \mathrm{~A}$ if, for every $\zeta_{1}$, the number $\Phi_{1}\left(S_{n}(e), \zeta_{1}\right)$ realizes $\left(\Omega_{2} \cdots \Omega_{n} \mathrm{~A}\right) *$. This condition will be satisfied if, for every $\zeta_{1}, \zeta_{2}, \Phi_{1}^{2}\left(S_{n}(e), \zeta_{1}, \zeta_{2}\right)$ realizes $\left(\Omega_{3} \cdots \Omega_{n} \mathrm{~A}\right)^{*}$. We continue this reduction, until finally the realization of $\Omega_{1} \cdots \Omega_{n} \mathrm{~A}$ by $S_{n}(e)$ is brought to depend only upon the realization of $A^{*}$ by the number $\Phi_{1}^{n}\left(S_{n}(e), \zeta_{1}, \cdots, \zeta_{n}\right)$ for every $\zeta_{1}, \cdots, \zeta_{n}$. The hypothesis of the lemma asserts that $\Phi_{n}\left(e, \zeta_{1}, \cdots, \zeta_{n}\right)$ realizes $\mathrm{A}^{*}$, but we have already observed, following the definition of $S_{n}(e)$, that in the event that $\Phi_{n}\left(e, \zeta_{1}, \cdots, \zeta_{n}\right)$ is a number, $\Phi_{1}^{n}\left(S_{n}(e), \zeta_{1}, \cdots, \zeta_{n}\right)=\Phi_{n}\left(e, \zeta_{1}, \cdots, \zeta_{n}\right)$ (cf. Definition $\mathrm{n})$.

COROLlaRY. If, for every choice of $\zeta_{1}, \cdots, \zeta_{n}$, the formula $\mathrm{A}^{*}$ is realized by $\phi\left(t_{1}, \cdots, t_{r}\right)$, where each $t_{i}$ is either some one of $\zeta_{1}, \cdots, \zeta_{n}$ or some prescribed constant or an arbitrary parameter, and if $\lambda w_{1} \cdots w_{r} \phi\left(w_{1}, \cdots, w_{r}\right)$ is a partial recursive function, then $\Omega_{1} \cdots \Omega_{n} \mathrm{~A}$ is realizable.

We choose for the number $e$ of the lemma the Gödel number of the function $\lambda \zeta_{1} \cdots \zeta_{n} U_{1}^{n+1}\left(\phi\left(t_{1}, \cdots, t_{r}\right), \zeta_{1}, \cdots, \zeta_{n}\right)$. We abbreviate this expression by writing " $\lambda \zeta_{1} \cdots \zeta_{n} \phi\left(t_{1}, \cdots, t_{r}\right)$."

Theorem 1. Every formula deducible by Postulates 1-18 and Postulate 23 (the intuitionistic predicate calculus with equality, and the Peano axioms) from arbitrary realizable formulas is realizable.

To prove the theorem it will be sufficient to show that each of the axioms 
is realizable; and, for the rules of inference, that the conclusion is realizable if the premise or premises are realizable.

In the discussion of the theorem we assume that the symbolism is subject to the restrictions that were stated in the explanation of the list of postulates. In addition, unless other free variables appear, it is assumed that each formula of the form of one of the axiom schemata contains, in order of appearance in the formula, exactly the free variables $a_{1}, \cdots, a_{k}$. A formula followed by an asterisk abbreviates the result of replacing those of the free variables $a_{1}, \cdots, a_{k}$ which appear in the formula by the respective numerals $\boldsymbol{n}_{1}, \cdots, n_{k}$.

In all of the cases we must consider formulas of the form $\Omega_{1} \ldots \Omega_{n} \mathrm{~A}$. For consideration of these cases we apply the corollary to Lemma 4 . Following the comments on each case we shall write in parentheses the choice of $\lambda \zeta_{1} \cdots \zeta_{n} \phi\left(t_{1}, \cdots, t_{r}\right)$. This indication will determine unambiguously the choice of variables $a_{\mu_{1}}, \cdots, a_{\mu_{j}}$ and formulas $A_{\nu_{1}}, \cdots, A_{\nu_{k}}$ as well (cf. remarks preceding Lemma 3 , and Lemma 4 and corollary). We proceed to the consideration of the separate cases.

(1a) $\mathrm{A} \supset(\mathrm{B} \supset \mathrm{A})$. Suppose that $a$ realizes $\mathrm{A}^{*}$ and that $b$ realizes $\mathrm{B}^{*}$, then $U_{1}^{1}(a)$ realizes $\mathrm{A}^{*}$. If we choose $U_{1}^{1}(a)$ to be $\phi\left(t_{1}, \cdots, t_{r}\right)$ in the corollary to Lemma 4 , and choose $n_{1}, \cdots, n_{k}, a, b$, to be $\zeta_{1}, \cdots, \zeta_{n}$, we obtain the desired result for a formula of the form (1a).

$$
\left(\lambda n_{1} \cdots n_{k} a b U_{1}^{1}(a)\right) \text {. }
$$

(1b) $(\mathrm{A} \supset(\mathrm{B} \supset \mathrm{C})) \supset((\mathrm{A} \supset \mathrm{B}) \supset(\mathrm{A} \supset \mathrm{C}))$. If $p$ realizes $(\mathrm{A} \supset(\mathrm{B} \supset \mathrm{C}))^{*}$ and $a$ realizes $A^{*}$, then by Lemma $3,(B \supset C) *$ is realized by $\Phi_{1}(p, a)$. If furthermore $q$ realizes $(\mathrm{A} \supset \mathrm{B})^{*}$, again by Lemma $3, \Phi_{1}(q, a)$ realizes $\mathrm{B}^{*}$. Thus $\mathrm{C}^{*}$ is realized by $\Phi_{1}\left(\Phi_{1}(p, a), \Phi_{1}(q, a)\right)$. Again the case is completed by application of the corollary to Lemma 4 taking $\zeta_{1}, \cdots, \zeta_{n}$ as $n_{1}, \cdots, n_{k}, p, q, a$.

$$
\begin{gathered}
\left(\lambda n_{1} \cdots n_{k} p q a \Phi_{1}\left(\Phi_{1}(p, a), \Phi_{1}(q, a)\right)\right) . \\
\frac{\mathrm{A}\left(\mathrm{a}_{1}, \cdots, \mathrm{a}_{k}\right), \mathrm{A}\left(\mathrm{a}_{1}, \cdots, \mathrm{a}_{k}\right) \supset \mathrm{B}\left(\mathrm{a}_{v_{1}}, \cdots, \mathrm{a}_{v_{l}}\right)}{\mathrm{B}\left(\mathrm{a}_{v_{1}}, \cdots, \mathrm{a}_{v_{l}}\right)} .
\end{gathered}
$$

Assume that the only free variables which appear in the formulas are the ones displayed and that they appear in the order listed. Then assume that $\mathrm{A}\left(\mathrm{a}_{1}, \cdots, \mathrm{a}_{k}\right)$ is realized by $a$ and that $\mathrm{A}\left(\mathrm{a}_{1}, \cdots, \mathrm{a}_{k}\right) \supset \mathrm{B}\left(\mathrm{a}_{v_{1}}, \cdots, \mathrm{a}_{v_{l}}\right)$ is realized by $c$. Then by Lemma $3, \mathrm{~A}\left(\boldsymbol{n}_{1}, \cdots, \boldsymbol{n}_{k}\right)$ is realized by $\Phi_{1}^{k}\left(a, n_{1}, \cdots, n_{k}\right)$, and $\mathrm{A}\left(n_{1}, \cdots, n_{k}\right) \supset \mathrm{B}\left(n_{p_{1}}, \cdots, n_{\eta_{l}}\right)$ is realized by $\Phi_{1}^{k+j}\left(c, n_{1}, \cdots, n_{k}, n_{\mu_{1}}, \cdots, n_{\mu_{j}}\right)$ where $\mathrm{a}_{\mu_{1}}, \cdots, \mathrm{a}_{\mu_{j}}$ are the variables of $\mathrm{B}\left(\mathrm{a}_{\nu_{1}}, \cdots, \mathrm{a}_{\nu_{l}}\right)$ in order of first appearance which are not among $\mathrm{a}_{1}, \cdots, \mathrm{a}_{k}$. Then, again by Lemma 3 , the formula $\mathrm{B}\left(\boldsymbol{n}_{\boldsymbol{p}_{1}}, \cdots, \boldsymbol{n}_{\boldsymbol{v}_{l}}\right)$ is realized by the number 


$$
\Phi_{1}\left(\Phi_{1}^{k+j}\left(c, n_{1}, \cdots, n_{k}, n_{\mu_{1}}, \cdots, n_{\mu_{j}}\right), \Phi_{1}^{k}\left(a, n_{1}, \cdots, n_{k}\right)\right) .
$$

This last expression is taken as $\phi\left(t_{1}, \cdots, t_{r}\right)$ in the corollary to Lemma 4 . Note that in this case arbitrary parameters and constants appear among the $t_{i} ; \zeta_{1}, \cdots, \zeta_{n}$ are chosen as $n_{v_{1}}, \cdots, n_{\nu_{l}}$.

$$
\left(\lambda n_{\nu_{1}} \cdots n_{\nu_{l}} \Phi_{1}\left(\Phi_{1}^{k+i}\left(c, n_{1}, \cdots, n_{k}, n_{\mu_{1}}, \cdots, n_{\mu_{j}}\right), \Phi_{1}^{k}\left(a, n_{1}, \cdots, n_{k}\right)\right)\right) .
$$

(3) $\mathrm{A} \supset(\mathrm{B} \supset \mathrm{A} \& \mathrm{~B})$. Suppose that $a$ realizes $\mathrm{A}^{*}$ and that $b$ realizes $\mathrm{B}^{*}$, then $(A \& B) *$ is realized by $2^{a} \cdot 3^{b}$.

$$
\left(\lambda n_{1} \cdots n_{k} a b 2^{a} \cdot 3^{b}\right) \text {. }
$$

(4a) A \& B DA. If $c$ realizes (A \& B)*, then $c$ is of the form $2^{a} \cdot 3^{b}$ and $a$ realizes $A^{*}$. Thus $(c)_{0}$ realizes $A^{*}$.

$$
\left(\lambda n_{1} \cdots n_{k} c(c)_{0}\right) \text {. }
$$

(4b) A \& B DB. If $c$ realizes (A \& B)*, then $(c)_{1}$ realizes $\mathrm{B}^{*} .\left(\lambda n_{1} \cdots n_{k} c(c)_{1}\right)$. (5a) $\mathrm{A} \supset \mathrm{A} \vee \mathrm{B}$. If $a$ realizes $\mathrm{A}^{*}$, then $2^{0} \cdot 3^{a}$ realizes $(\mathrm{A} \vee \mathrm{B})^{*}$.

$$
\left(\lambda n_{1} \cdots n_{k} a 2^{0} \cdot 3^{b}\right) \text {. }
$$

(5b) $\mathrm{B} \supset \mathrm{A} \vee \mathrm{B}$. If $b$ realizes $\mathrm{B}^{*}$, then $2^{1} \cdot 3^{b}$ realizes $(\mathrm{A} \vee \mathrm{B})^{*}$.

$$
\left(\lambda n_{1} \cdots n_{k} b 2^{1} \cdot 3^{a}\right) \text {. }
$$

(6) $(\mathrm{A} \supset \mathrm{C}) \supset((\mathrm{B} \supset \mathrm{C}) \supset(\mathrm{A} \vee \mathrm{B} \supset \mathrm{C}))$. Suppose that $h$ realizes $(\mathrm{A} \supset \mathrm{C})^{*}$ and that $m$ realizes $(\mathrm{B} \supset \mathrm{C})^{*}$. In addition, let us suppose that $k$ realizes $(\mathrm{A} \vee \mathrm{B})^{*}$. Then there are two possibilities as $(k)_{0}=0$ or $(k)_{0}=1$. We shall show that in either case $C^{*}$ is realized by $\beta\left((k)_{0}\right) \cdot \Phi_{1}\left(h,(k)_{1}\right)+(k)_{0} \cdot \Phi_{1}\left(m,(k)_{1}\right)$. If $(k)_{0}=0$, then $(k)_{1}$ must realize $\mathrm{A}^{*}$, and $\Phi_{1}\left(h,(k)_{1}\right)$ realizes $\mathrm{C}^{*}$; recalling that $\beta(0)=1$, we have the desired result. If $(k)_{0}=1$, then $(k)_{1}$ must realize $\mathrm{B}^{*}$, and $\Phi_{1}\left(m,(k)_{1}\right)$ realizes $C^{*}$; in this case we have $\beta(1)=0$.

$$
\left(\lambda n_{1} \cdots n_{k} h m k \beta\left((k)_{0}\right) \cdot \Phi_{1}\left(h,(k)_{1}\right)+(k)_{0} \cdot \Phi_{1}\left(m,(k)_{1}\right)\right) .
$$

(7) $(\mathrm{A} \supset \mathrm{B}) \supset((\mathrm{A} \supset>\mathrm{B}) \supset>\mathrm{A})$. In consideration of this schema, we observe first that a formula $7 \mathrm{~A}^{*}$ is realized by the Gödel number of any partial recursive function, thus by any natural number, if and only if $A^{*}$ is not realizable. Now let us suppose that $(A \supset B)^{*}$ is realized by $p$ and that $(A \supset>B) *$ is realized by $q$; then if, in addition, $\mathrm{A}^{*}$ were realized by $a$, the formula $\mathrm{B}^{*}$ would be realized by $\Phi_{1}(p, a)$ and $7 \mathrm{~B}^{*}$ would be realized by $\Phi_{1}(q, a)$. But this is impossible $[15, \S 7,(c)]$. Thus if $(A \supset B)^{*}$ and $(A \supset>B) *$ are both realizable, $A^{*}$ is not realizable, and $7 A^{*}$ is realized by any number, say $0(q)$.

$$
\left(\lambda n_{1} \cdots n_{k} p q 0(q)\right) \text {. }
$$

(8) $7 \mathrm{~A} \supset(\mathrm{A} \supset \mathrm{B})$. The reasoning in this case is somewhat like that in the 
preceding one. Not both $7 \mathrm{~A}^{*}$ and $\mathrm{A}^{*}$ can be realizable. Thus if $c$ realizes $7 \mathrm{~A}^{*}$, the formula $(\mathrm{A} \supset \mathrm{B})^{*}$ is realized by any number, say $0(c)$.

$$
\begin{gathered}
\left(\lambda n_{1} \cdots n_{k} c 0(c)\right) . \\
\frac{\mathrm{C} \supset \mathrm{A}(\mathrm{b})}{\mathrm{C} \supset \mathrm{VxA}(\mathrm{x})} .
\end{gathered}
$$

Suppose a formula above the line contains, in order of appearance, exactly the free variables $a_{1}, \cdots, a_{j}, b, a_{j+1}, \cdots, a_{k}$, then the formula below the line will contain, in order of appearance, exactly the free variables $a_{1}, \cdots, a_{j}, a_{j+1}, \cdots, a_{k}$, cf. restriction on Rule 9.

Let us assume that $p$ realizes the formula above the line. Let any choice be made of the numerals $\boldsymbol{n}_{\mathbf{1}}, \cdots, \boldsymbol{n}_{j}, \boldsymbol{n}, \boldsymbol{n}_{j+1}, \cdots, \boldsymbol{n}_{k}$; after this has been done, let any $c$ be chosen which realizes $C^{*}$; then $\Phi_{1}^{k+2}\left(p, n_{1}, \cdots, n_{j}, n, n_{j+1}, \cdots, n_{k}, c\right)$ realizes $(A(n))^{*}$. Now observe that, since $b$ did not occur in $C$, the choice of $c$ did not depend on the choice of $n$. Thus for any choice of $n_{1}, \cdots, n_{j}, n_{j+1}, \cdots, n_{k}$, followed by any choice of $c$, and then any choice of $n$, the formula $(\mathrm{A}(n))^{*}$ is realized by $\Phi_{1}^{k+2}\left(p, n_{1}, \cdots, n_{j}, n, n_{j+1}, \cdots, n_{k}, c\right)$. Thus if we interpret this expression as $\phi\left(t_{1}, \cdots, t_{k}\right)$ and interpret $n_{1}, \cdots, n_{j}, n_{j+1}, \cdots, n_{k}, c, n$ as $\zeta_{1}, \cdots, \zeta_{n}$ in the corollary to Lemma 4, we may demonstrate that the formula below the line is realizable.

$$
\left(\lambda n_{1} \cdots n_{j}, n_{j+1} \cdots n_{k} c n \Phi_{1}^{k+2}\left(p, n_{1}, \cdots, n_{j}, n, n_{j+1}, \cdots, n_{k}, c_{)}\right)\right.
$$

(10) $\operatorname{VxA}(\mathrm{x}) \supset \mathrm{A}\left(\mathrm{t}\left(\mathrm{a}_{p_{1}}, \cdots, \mathrm{a}_{v_{j}}\right)\right)$. Suppose that those of the free variables $a_{1}, \cdots, a_{k}$ which occur in $t\left(a_{p_{1}}, \cdots, a_{v_{j}}\right)$ are exactly the ones which are exhibited, and suppose that $t\left(a_{v_{1}}, \cdots, a_{v_{j}}\right)$ represents the general recursive function $\lambda x_{p_{1}} \cdots x_{v_{j}} t\left(x_{v_{1}}, \cdots, x_{v_{j}}\right)$, cf. Lemma 1 , and that $t\left(n_{v_{1}}, \cdots, n_{v_{j}}\right)$ $=n$. Now, if $c$ realizes $(\mathrm{VxA}(\mathrm{x}))^{*}$, then $\Phi_{1}(c, n)$ realizes $(\mathrm{A}(n))^{*}$.

By Lemma $2, \Phi_{1}(c, n)$ also realizes $\left(\mathrm{A}\left(\mathrm{t}\left(\boldsymbol{n}_{p_{1}}, \cdots, n_{v_{j}}\right)\right)\right)^{*}$. Finally, $\left(\mathrm{A}\left(\mathrm{t}\left(n_{v_{1}}, \cdots, n_{v_{j}}\right)\right)\right)^{*}$ is realized by $\Phi_{1}\left(c, t\left(n_{\eta_{1}}, \cdots, n_{v_{j}}\right)\right)$.

$$
\left(\lambda n_{1} \cdots n_{k} c \Phi_{1}\left(c, t\left(n_{v_{1}}, \cdots, n_{v_{j}}\right)\right)\right) \text {. }
$$

(11) $\mathrm{A}\left(\mathrm{t}\left(\mathrm{a}_{v_{1}}, \cdots, \mathrm{a}_{v_{j}}\right)\right) \supset \mathrm{HxA}(\mathrm{x})$. Assume that the conditions stated in the first sentence of $(10)$ are satisfied. Then, if $c$ realizes $\left(\mathrm{A}\left(\mathrm{t}\left(\boldsymbol{n}_{\boldsymbol{p}_{1}}, \cdots, \boldsymbol{n}_{\boldsymbol{v}_{j}}\right)\right)\right)^{*}$, again by Lemma $2, c$ will also realize $(\mathrm{A}(\boldsymbol{n}))^{*}$. Thus the formula $(\operatorname{GxA}(\mathrm{x}))^{*}$ will be realized by $E_{2}\left(t\left(n_{p_{1}}, \cdots, n_{v_{j}}\right), c\right)$.

$$
\begin{gathered}
\left(\lambda n_{1} \cdots n_{k} c E_{2}\left(t\left(n_{v_{1}}, \cdots, n_{p_{j}}\right), c\right)\right) . \\
\frac{\mathrm{A}(\mathrm{b}) \supset \mathrm{C}}{\mathrm{H \times A}(\mathrm{x}) \supset \mathrm{C}} .
\end{gathered}
$$

Suppose a formula above the line contains, in order of appearance, ex- 
actly the free variables $a_{1}, \cdots, a_{j}, b, a_{j+1}, \cdots, a_{k}$; then the formula below the line will contain, in order of appearance, exactly the free variables $a_{1}, \cdots, a_{j}, a_{j+1}, \cdots, a_{k}$, cf. restriction on Rule 12 . For any choice of numerals $n_{1}, \cdots, n_{j}, n, n_{j+1}, \cdots, n_{k}$, if $p$ realizes $(\mathrm{A}(\boldsymbol{n}) \supset \mathrm{C})^{*}$ and $m$ realizes $(\mathrm{A}(n))^{*}$, then $\mathrm{C}^{*}$ will be realized by $\Phi_{1}^{k+2}\left(p, n_{1}, \cdots, n_{j}, n, n_{j+1}, \cdots, n_{k}, m\right)$. Now let us suppose that the formula $(\operatorname{GxA}(\mathrm{x}))^{*}$ is realized by $h$ and that $(h)_{0}=l$, then the formula $(\mathrm{A}(I))^{*}$ will be realized by $(h)_{1}$. Since C does not contain the free variable $b$, the formula $C^{*}$ will be realized by $\Phi_{1}^{k+2}\left(p, n_{1}, \cdots, n_{j},(h)_{0}, n_{j+1}, \cdots, n_{k},(h)_{1}\right)$, for the choice of $n$ might have been $l$, and the consequent choice of $m$ might have been $(h)_{1}$.

$$
\left(\lambda n_{1} \cdots n_{k} h \Phi_{1}^{k+2}\left(p, n_{1}, \cdots, n_{j},(h)_{0}, n_{j+1}, \cdots, n_{k},(h)_{1}\right)\right) .
$$

(13) $\mathrm{A}(0) \& \forall \mathrm{x}\left(\mathrm{A}(\mathrm{x}) \supset \mathrm{A}\left(\mathrm{x}^{\prime}\right)\right) \supset \mathrm{A}(\mathrm{b})$. As a preliminary step for this case we define recursively a function $\lambda x z \rho(x, z)$ by the following equations: $\rho(0, z)=(z)_{0}, \rho\left(x^{\prime}, z\right)=\Phi_{1}^{2}\left((z)_{1}, x, \rho(x, z)\right)$. Now let us assume that the formula $\left(\mathrm{A}(0) \& \forall \mathrm{x}\left(\mathrm{A}(\mathrm{x}) \supset \mathrm{A}\left(\mathrm{x}^{\prime}\right)\right)\right)^{*}$ is realized by $a$, then we shall prove by induction that $\mathrm{A}(n)^{*}$, for any choice of $n$, is realized by $\rho(n, a)$. For the basis, we observe that $(a)_{0}$ realizes $(\mathrm{A}(0))^{*}$, but $\rho(0, a)=(a)_{0}$. For the induction step, let us assume that $\rho(n, a)$ realizes $(\mathrm{A}(n))^{*}$; then, by Lemma 3 and the first hypothesis of this case, $\Phi_{1}^{2}\left((a)_{1}, n, \rho(n, a)\right)$ realizes $\left(\mathrm{A}\left(n^{\prime}\right)\right)^{*}$. Thus $\rho\left(n^{\prime}, a\right)$ realizes $\left(\mathrm{A}\left(\boldsymbol{n}^{\prime}\right)\right)^{*}$. Finally, we note that $\mathrm{b}$ is the last free variable in order of appearance in a formula of the form of Schema 13. $\left(\lambda n_{1} \cdots n_{k} n a \rho(n, a)\right)$.

(14) $\mathrm{a}^{\prime}=\mathrm{b}^{\prime} \supset \mathrm{a}=\mathrm{b}$. If $p$ realizes $\boldsymbol{n}^{\prime}=\boldsymbol{m}^{\prime}$, then $0(p)$ realizes $\boldsymbol{n}=\boldsymbol{m}$. $(\lambda n m p 0(p))$.

(15) $\mathrm{a}^{\prime}=0$. Since for every $n, n^{\prime} \neq 0,7 n^{\prime}=0$ is realized by any number, say $0(n) .(\lambda n 0(n))$.

(16) $\mathrm{a}=\mathrm{a}$. The formula $n=n$ is realized by $0(n) .\left(\lambda_{n} 0(n)\right)$.

(17) $\mathrm{a}=\mathrm{b} \supset(\mathrm{a}=\mathrm{c} \supset \mathrm{b}=\mathrm{c})$. If $p$ realizes $n=m$, and $q$ realizes $n=k$, then $0(q)$ realizes $\boldsymbol{m}=k$. $(\lambda n m k p q 0(q))$.

(18) $\mathrm{a}=\mathrm{b} \supset \mathrm{a}^{\prime}=\mathrm{b}^{\prime}$. If $p$ realizes $\boldsymbol{n}=\boldsymbol{m}$, then $0(p)$ realizes $\boldsymbol{n}^{\prime}=\boldsymbol{m}^{\prime}$. $(\lambda n m p 0(p))$.

(23) $\mathrm{b}=c \supset(\mathrm{A}(\mathrm{b}) \supset \mathrm{A}(\mathrm{c}))$. If $p$ realizes $\boldsymbol{n}=\boldsymbol{m}$, and $q$ realizes $(\mathrm{A}(\boldsymbol{n}))^{*}$, then $U_{1}^{1}(q)$ realizes $(\mathrm{A}(\boldsymbol{m}))^{*} .\left(\lambda n m n_{1} \cdots n_{k} p q U_{1}^{1}(q)\right)$.

COROLLARY 1.1. Every formula deducible from arbitrary realizable formulas in a formal system containing Postulates 1-18, 23 and any axioms having the form of equations defining general recursive functions is reálizable. In particular, every formula provable in $S_{1}$ is realizable.

Any formula $\mathrm{A}\left(\mathrm{a}_{1}, \cdots, \mathrm{a}_{k}\right)$ containing the free variables in indicated order of appearance which has the form of a recursion equation is an elementary formula and is realized by $S_{k}(e)$, where $e$ defines $\lambda x_{1} \cdots x_{k} U_{1}^{k+1}\left(0, x_{1}\right.$, 
$\left.\cdots, x_{k}\right)$, cf. Lemma 4 . This observation along with Theorem 1 completes the proof of the corollary.

Corollary 1.1 can be extended to systems containing predicate symbols which represent general recursive predicates $\left(^{6}\right)$, with axioms which either define the predicates explicitly from the functions or are constructed from the recursion equations of the latter by the usual device for eliminating function symbols in terms of predicate symbols [10, vol. 1, pp. 460-461].

Suppose, for example, that a function $\mathrm{f}$ is defined by recursion equations

$$
\begin{aligned}
f(0, b) & =g(b), \\
f\left(a^{\prime}, b\right) & =h(f(a, b), a, b) .
\end{aligned}
$$

And suppose that axioms have already been introduced for elementary formulas $\mathrm{G}(\mathrm{b}, \mathrm{c})$ and $\mathrm{H}(\mathrm{d}, \mathrm{a}, \mathrm{b}, \mathrm{c})$ representing the respective predicates $\mathrm{g}(\mathrm{b})=c$ and $h(d, a, b)=c$. Then the following axioms serve to introduce $\mathrm{F}(\mathrm{a}, \mathrm{b}, \mathrm{c})$ representing the predicate $f(a, b)=c$ :

$$
\mathrm{H} x(\mathrm{~F}(0, \mathrm{~b}, \mathrm{x}) \& \mathrm{G}(\mathrm{b}, \mathrm{x})) \text {, }
$$

$$
\operatorname{HxHy}\left(F\left(a^{\prime}, b, x\right) \& H(y, a, b, x) \& F(a, b, y)\right) \text {. }
$$

For every $n$ the formula $\operatorname{\exists x}(F(0, n, \mathrm{x}) \& \mathrm{G}(n, \mathrm{x}))$ is realized by $E_{3}(g(n), 0,0)$. For every $n$ and $m$ the formula $\operatorname{Hx} \exists y\left(\mathrm{~F}\left(\boldsymbol{n}^{\prime}, \boldsymbol{m}, \mathrm{x}\right) \& \mathrm{H}(\mathrm{y}, \boldsymbol{n}, \boldsymbol{m}, \mathrm{x})\right.$ $\& \mathrm{~F}(\boldsymbol{n}, \boldsymbol{m}, \mathrm{y}))$ is realized by $E_{5}\left(f\left(n^{\prime}, m\right), f(n, m), 0,0,0\right)$. If $g$ and $h$ are general recursive, formulas (i) and (ii) are realizable.

CoROLlaRY 1.2. For every provable formula of $S_{1}$ containing no free variables and of the form $\forall \mathrm{x}_{1} \cdots \forall \mathrm{x}_{k} \mathrm{H} \mathrm{A}\left(\mathrm{x}_{1}, \cdots, \mathrm{x}_{k}, \mathrm{y}\right)$, there exists a general recursive function $\phi\left(x_{1}, \cdots, x_{k}\right)$ such that for every $n_{1}, \cdots, n_{k}$, if $\phi\left(n_{1}, \cdots, n_{k}\right)=n$, then $\mathrm{A}\left(\boldsymbol{n}_{1}, \cdots \cdot \boldsymbol{n}_{k}, \boldsymbol{n}\right)$ is a realizable formula.

By Corollary 1.1, a provable formula is realizable. Let the number $u$ be a realization for the formula of the form $\forall x_{1} \cdots \forall x_{k} \exists y A\left(x_{1}, \cdots, x_{k}, y\right)$. Then, by Lemma 3 , the number $\Phi_{1}^{k}\left(u, n_{1}, \cdots, n_{k}\right)$ realizes $\operatorname{ByA}\left(n_{1}, \cdots, n_{k}, \mathrm{y}\right)$ for any set of natural numbers $n_{1}, \cdots, n_{k}$. By the definition of realizability for a formula of this form, the number $\left(\Phi_{1}^{k}\left(u, n_{1}, \cdots, n_{k}\right)\right)_{1}$ realizes the formula $\mathrm{A}\left(\boldsymbol{n}_{1}, \cdots, \boldsymbol{n}_{k}, \boldsymbol{n}\right)$ where $\left(\Phi_{1}^{k}\left(u, n_{1}, \cdots, n_{k}\right)\right)_{0}=n$. The function $\lambda x_{1} \cdots x_{k}\left(\Phi_{1}^{k}\left(u, x_{1}, \cdots, x_{k}\right)\right)_{0}$ is general recursive since $\Phi_{1}^{k}\left(u, n_{1}, \cdots, n_{k}\right)$ is defined for each set $n_{1}, \cdots, n_{k}$ of natural numbers.

2. Realizability of $A \supset \AA A$ and $® A \supset A$. In this section the formal symbolism may be that of $S_{1}$, or of a related system with some other selection of predicate and function symbols representing recursive predicates and functions.

Kleene has observed that if, under the usual interpretations, the recursive predicates $T_{1}(c, a, b)$ and $a=b$, and either the two recursive functions $2^{a} \cdot 3^{b}$

(6) Including systems of type $B, D$, and $E$ in the classification of $[15, \S 4]$. 
and $U(a)$ or corresponding predicates $2^{a} \cdot 3^{b}=c$ and $U(a)=c$, are expressible in the formalism, then for each formula $A$ there exist formulas a $\AA A$ and $\AA A$ which express the intuitive predicates " $a$ realizes $A$ " and " $\mathrm{A}$ is realizable," respectively $[15, \S 12]$. Kleene gives a definition of a $\AA A$, supposing the function $U(a)$ is expressible, which we modify by the following changes for the situation in which only the predicate $U(a)=c$ is expressible. For the formulas which represent the three intuitive predicates $T_{1}(c, a, b), 2^{a} \cdot 3^{b}=c$, and $U(a)=c$ we write $\mathrm{T}(\mathrm{c}, \mathrm{a}, \mathrm{b}), \mathrm{E}(\mathrm{a}, \mathrm{b}, \mathrm{c})$, and $\mathrm{U}(\mathrm{a}, \mathrm{c})$, respectively. Then for $\mathrm{a}$ formula of the form $U(a) \AA A$, we have $\exists y(U(a, y) \& y \circledast A)$.

Two cases may now be distinguished. Either the symbolism affords $\mathrm{T}(\mathrm{c}, \mathrm{a}, \mathrm{b}), \mathrm{E}(\mathrm{a}, \mathrm{b}, \mathrm{c})$, and $\mathrm{U}(\mathrm{a}, \mathrm{c})$ as elementary formulas, or does not. In the first case $\left({ }^{7}\right)$, the two following propositions will hold as direct consequences of the clause of the definition of realizability for an elementary formula without free variables (the partial recursive functions being simply the constant function 0 of the proper number of arguments):

(1) For natural numbers $n, m, k, \mathrm{~T}(\boldsymbol{k}, \boldsymbol{n}, \boldsymbol{m}), \mathrm{E}(\boldsymbol{n}, \boldsymbol{m}, \boldsymbol{k})$, or $\mathrm{U}(\boldsymbol{n}, \boldsymbol{k})$ is realizable if, and only if, $T_{1}(k, n, m), 2^{n} \cdot 3^{m}=k$, or $U(n)=k$, respectively.

(2) There exist partial recursive functions $\lambda z x y \tau(z, x, y), \lambda x y z \epsilon(x, y, z)$, and $\lambda x z v(x, z)$ such that for natural numbers $n, m, k$, if $\mathrm{T}(\boldsymbol{k}, \boldsymbol{n}, \boldsymbol{m}), \mathrm{E}(\boldsymbol{n}, \boldsymbol{m}, \boldsymbol{k})$, or $\mathrm{U}(\boldsymbol{n}, \boldsymbol{k})$ is realizable, then $\tau(k, n, m), \epsilon(n, m, k)$, or $\mathrm{v}(n, k)$, respectively, realizes it.

For the second case $\left({ }^{8}\right)$, we can make use of the following two lemmas to prove the existence of $\mathrm{T}(\mathrm{c}, \mathrm{a}, \mathrm{b}), \mathrm{E}(\mathrm{a}, \mathrm{b}, \mathrm{c})$, and $\mathrm{U}(\mathrm{a}, \mathrm{c})$ representing the three intuitive predicates, and having the two stated properties, when the formalism contains at least the functions and predicate ', $\cdot,+,=$ of $S_{1}$, or instead the four predicates $a^{\prime}=c,=, a+b=c, a \cdot b=c$. Although the result is one which concerns only the formation rules, and does not relate to any set of deductive postulates, it will be convenient to prove it by making use of known properties of the deductive system $S_{1}$, including, for the proof that $\mathrm{T}(\mathrm{c}, \mathrm{a}, \mathrm{b})$, $\mathrm{E}(\mathrm{a}, \mathrm{b}, \mathrm{c})$, and $\mathrm{U}(\mathrm{a}, \mathrm{c})$ represent the intuitive predicates, the fact that under the usual interpretations only true formulas can be provable in $S_{1}$.

Lemma 5. For every primitive recursive predicate $R\left(a_{1}, \cdots, a_{k}\right)$ of exactly the variables exhibited, there exists a formula $\mathrm{R}\left(\mathrm{a}_{1}, \cdots, \mathrm{a}_{k}\right)$ containing exactly the free variables exhibited, and such that, for natural numbers $n_{1}, \cdots, n_{k}$, if $R\left(n_{1}, \cdots, n_{k}\right)$, then $\mathrm{R}\left(n_{1}, \cdots, n_{k}\right)$ is provable in $S_{1}$, and if $\bar{R}\left(n_{1}, \cdots, n_{k}\right)$, then $7 \mathrm{R}\left(\boldsymbol{n}_{1}, \cdots, \boldsymbol{n}_{k}\right)$ is provable in $S_{1}$. Furthermore, there exists a partial recursive function $\lambda x_{1} \cdots x_{k} \rho\left(x_{1}, \cdots, x_{k}\right)$ such that $\rho\left(n_{1}, \cdots, n_{k}\right)$ realizes $\mathrm{R}\left(\boldsymbol{n}_{1}, \cdots, \boldsymbol{n}_{k}\right)$ if that formula is realizable.

The proof of this lemma is given in $\$ 4$ (Part II), where it is stated as Corollary 9.1.

(7) For example $[15, \S 4$, Plans C-E].

(8) For example $[15, \S 4$, Plans A and B]. 
LemMA 6. If $\mathrm{R}\left(\mathrm{a}_{1}, \cdots, \mathrm{a}_{k}\right)$ is the formula which corresponds to the recursive predicate $R\left(a_{1}, \cdots, a_{k}\right)$ by Lemma 5 , then $\mathrm{R}\left(\boldsymbol{n}_{1}, \cdots, \boldsymbol{n}_{k}\right)$ is realizable if and only if $R\left(n_{1}, \cdots, n_{k}\right)$.

Since $R\left(a_{1}, \cdots, a_{k}\right)$ is general recursive, there exists an effective decision procedure for any set of arguments $n_{1}, \cdots, n_{k}$; thus we have $R\left(n_{1}, \cdots, n_{k}\right)$ or $\bar{R}\left(n_{1}, \cdots, n_{k}\right)$. If $\mathrm{R}\left(\boldsymbol{n}_{1}, \cdots, \boldsymbol{n}_{k}\right)$ is realizable, then $7 \mathrm{R}\left(\boldsymbol{n}_{1}, \cdots, \boldsymbol{n}_{k}\right)$ is not realizable, therefore, by Corollary 1.1, not provable, and we have from Lemma 5 not $\bar{R}\left(n_{1}, \cdots, n_{k}\right)$, thus $R\left(n_{1}, \cdots, n_{k}\right)$. Conversely, if $R\left(n_{1}, \cdots, n_{k}\right)$, then by Lemma 5 and Corollary 1.1 the formula $\mathrm{R}\left(\boldsymbol{n}_{1}, \cdots, \boldsymbol{n}_{k}\right)$ is realizable.

For any formal symbolism containing the formulas $\mathrm{T}(\mathrm{c}, \mathrm{a}, \mathrm{b}), \mathrm{E}(\mathrm{a}, \mathrm{b}, \mathrm{c})$ and $U(a, c)$ which represent the intuitive predicates, and have the two properties (1) and (2), we have the following theorem. The theorem will also hold for definitions of a $\circledast \mathrm{A}$ using an expression for the function $U(a)$ instead of for the predicate $U(a)=c$, the only difference in the proof for this situation being that, where applications of the properties (1) and (2) to $U(a, c)$ are made, Lemma 2 should instead be applied to interchange $U(n) \circledast A$ with $m \circledast A$ where $m=U(n)$.

THEOREM 2. If the formula A does not contain the free variable a, the formulas $\mathrm{a} \circledast \mathrm{A} \supset \mathrm{A}$ and $\mathrm{A} \supset \mathrm{H} \times(\mathrm{x} \circledast \mathrm{A})$ are realizable.

Assume that A contains exactly the free variables $a_{1}, \cdots, a_{k}$, and that $\mathrm{A}(\mathrm{b})$ contains the additional free variable $\mathrm{b}$, and, in addition, that the free variables occur in the order $\mathrm{a}_{1}, \cdots, \mathrm{a}_{k}$ in the formulas $\mathrm{A}$ and $\mathrm{VxA}(\mathrm{x})$.

To prove the theorem, we first demonstrate by induction on the number of logical symbols in $\mathrm{A}$ the following: For every $a$, if $m$ realizes $\mathrm{a} \AA \mathrm{A}^{*}$, then a realizes $\mathrm{A}^{*}$. For each formula $\mathrm{A}$, there exists a partial recursive function $\lambda x \rho(\mathrm{x})$, such that if a realizes $\mathrm{A}^{*}$ then $E_{2}(a, \rho(a))$ realizes $3 \mathrm{x}\left(\mathrm{x} \circledast \mathrm{A}^{*}\right)$.

After this fact has been established, the proof of the theorem will be completed by application of the corollary to Lemma 4 .

(i) For the two cases of the basis, let $\mathrm{A}^{*}$ be an elementary formula. Then $a ® \mathrm{~A}^{*}$ has the form $a=0 \& \mathrm{~A}^{*}$. If the number $m$ realizes $a=0 \& \mathrm{~A}^{*}$, then $a=0$ and $\mathrm{A}^{*}$ is realized by $a$.

For the second part of the basis, suppose that $a$ realizes $\mathrm{A}^{*}$. Then $a=0$ and $\exists \mathrm{x}\left(\mathrm{x}=0 \& \mathrm{~A}^{*}\right)$ is realized by $E_{3}(a, 0,0)=E_{2}(a, 1(a))$.

(ii) Let $\mathrm{A}$ be of the form $\mathrm{B} \& \mathrm{C}$. Suppose for the first part of the case that $m$ realizes $d \circledast\left(\mathrm{B}^{*} \& \mathrm{C}^{*}\right)$; this formula has the form $\exists \mathrm{x} \exists \mathrm{y}\left(\mathrm{E}(\mathrm{x}, \mathrm{y}, d) \& \mathrm{x} \circledast \mathrm{B}^{*}\right.$ $\& \mathrm{y}\left(C^{*}\right)$, and the number $m$ must be of the form $E_{5}(b, c, e, g, h)$, where $e$ realizes $\mathrm{E}(\boldsymbol{b}, \boldsymbol{c}, \boldsymbol{d}), \boldsymbol{g}$ realizes $\boldsymbol{b} \circledast \mathrm{B}^{*}$ and $h$ realizes $c{ }^{\circledR} \mathrm{C}^{*}$. Since the number $e$ realizes $\mathrm{E}(b, c, d)$, which represents the recursive relation $2^{b} \cdot 3^{c}=d$, we have by (1), $2^{b} \cdot 3^{c}=d$. Applying the induction hypothesis, $b$ realizes $\mathrm{B}^{*}$ and $c$ realizes $\mathrm{C}^{*}$. Thus $d$ realizes $\mathrm{B}^{*} \& \mathrm{C}^{*}$.

For the second part of this case, suppose that $d$ realizes $\mathrm{B}^{*} \& \mathrm{C}^{*}$, then 
$\mathrm{d}=2^{b} \cdot 3^{c}$, where $b$ realizes $\mathrm{B}^{*}$ and $c$ realizes $\mathrm{C}^{*}$. By the induction hypothesis, the formula $\exists \mathrm{x}\left(\mathrm{x} \circledast \mathrm{B}^{*}\right)$ is realized by $E_{2}(b, \phi(b))$, where $\phi(b)$ realizes $b \circledast \mathrm{B}^{*}$, and $\exists y\left(y @ C^{*}\right)$ is realized by $E_{2}(c, \sigma(c))$, where $\sigma(c)$ realizes $c ® C^{*}$. Thus the formula $\exists z\left(z @\left(B^{*} \& C^{*}\right)\right)$, which has the form $\exists z \exists x \exists y\left(E(x, y, z) \& x @ B^{*}\right.$ $\left.\& \mathrm{y} \circledast C^{*}\right)$, is realized by the number $E_{6}\left(2^{b} \cdot 3^{c}, b, c, \epsilon\left(b, c, 2^{b} \cdot 3^{c}\right), \phi(b), \sigma(c)\right)$, where, by $(2), \epsilon(k, l, m)$ is a realization of $\mathrm{E}(\boldsymbol{k}, \mathbf{l}, \boldsymbol{m})$ in case the latter is realizable, and where $2^{b} \cdot 3^{c}=d, b=(d)_{0}$, and $c=(d)_{1}$. Finally we note that, for every $j>2, E_{j}\left(x_{1}, x_{2}, \cdots, x_{j}\right)=E_{2}\left(x_{1}, E_{j-1}\left(x_{2}, \cdots, x_{j}\right)\right)$. This fact will be utilized at several points below in this demonstration.

(iii) Let $\mathrm{A}$ be of the form $\mathrm{B} \vee \mathrm{C}$. For the first part of this case, suppose that $m$ realizes $d \circledast\left(\mathrm{B}^{*} \vee \mathrm{C}^{*}\right)$. This formula has the form $\mathrm{Gx}\left(\mathrm{E}(0, \mathrm{x}, d) \& \mathrm{x} \circledast \mathrm{B}^{*}\right)$ $\bigvee \operatorname{By}\left(\mathrm{E}(1, \mathrm{y}, d) \& \mathrm{y}{ }^{\circledR} C^{*}\right)$, so that $m$ must be of the form $E_{4}(j, g, e, h)$, where either $j=0, e$ realizes $\mathrm{E}(0, \boldsymbol{\xi}, \boldsymbol{d})$, and $h$ realizes $\boldsymbol{\xi} \circledast \mathrm{B}^{*}$, or else $j=1, e$ realizes $\mathrm{E}(1, \boldsymbol{g}, \boldsymbol{d})$, and $h$ realizes $\boldsymbol{g} \circledast \mathrm{C}^{*}$. In the former case, by the induction hypothesis, $g$ realizes $\mathrm{B}^{*}$, and by (1), $d=2^{0} \cdot 3^{o}$. In the latter case, similarly, $g$ realizes $\mathrm{C}^{*}$ and $\mathrm{d}=2^{1} \cdot 3^{o}$. Thus $d=2^{j} \cdot 3^{o}$ realizes $\mathrm{B}^{*} \vee \mathrm{C}^{*}$.

Let us assume, for the second part of the case, that $d$ realizes $\mathrm{B}^{*} \vee \mathrm{C}^{*}$; then $d=2^{i} \cdot 3^{o}$, where $j=0$ and $g$ realizes $\mathrm{B}^{*}$, or $j=1$ and $g$ realizes $\mathrm{C}^{*}$. Thus either $j=0$ and some $\phi(g)=\phi\left((d)_{1}\right)$ realizes $\xi \circledast \mathrm{B}^{*}$, or $j=1$ and some $\sigma(g)=\sigma\left((d)_{1}\right)$ realizes $\boldsymbol{g}^{\circledR} \mathrm{C}^{*}$. In either case, using (2), the number $E_{4}(j, g, \epsilon(j, g, d), \beta(j) \cdot \phi(g)+j \cdot \sigma(g))$ realizes the formula $\exists \mathrm{x}\left(\mathrm{E}(0, \mathrm{x}, d) \& \mathrm{x} @ \mathrm{~B}^{*}\right)$ $\vee{ }^{\prime} \mathrm{y}\left(\mathrm{E}(1, \mathrm{y}, d) \& \mathrm{y}{ }^{\circledR} \mathrm{C}^{*}\right)$. Thus the formula $\exists \mathrm{z}\left(\exists \mathrm{x}\left(\mathrm{E}(0, \mathrm{x}, \mathrm{z}) \& \mathrm{x} \AA^{*}\right)\right.$ $\left.\bigvee \mathcal{B} y\left(\mathrm{E}(1, \mathrm{y}, \mathrm{z}) \& \mathrm{y}{ }^{\circledR} \mathrm{C}^{*}\right)\right)$ is realized by the number

$$
E_{5}\left(d,(d)_{0},(d)_{1}, \epsilon\left((d)_{0},(d)_{1}, d\right), \beta\left((d)_{0}\right) \cdot \phi\left((d)_{1}\right)+(d)_{0} \cdot \sigma\left((d)_{1}\right)\right) .
$$

(iv) Let $A$ be of the form $B \supset C$. For the first part of this case, assume that $m$ realizes a formula of the form $\forall x\left(x ® B^{*} \supset \exists y(T(d, x, y) \& \exists v(U(y, v)\right.$ $\left.\left.\& \mathrm{v}\left(C^{*}\right)\right)\right)$. We wish, then, to show that $d$ realizes $\mathrm{B}^{*} \supset \mathrm{C}^{*}$. The number $m$ must be such that if $l$ realizes $b \circledast \mathrm{B}^{*}$, then, by Lemma $3, \Phi_{1}^{2}(m, b, l)$ realizes $\exists \mathrm{y}\left(\mathrm{T}(d, b, \mathrm{y}) \& \exists \mathrm{v}\left(\mathrm{U}(\mathrm{y}, \mathrm{v}) \& \mathrm{v} \circledast C^{*}\right)\right)$. Now, suppose that $b$ realizes $\mathrm{B}^{*}$. The induction hypothesis states that there exists a partial recursive function $\lambda x \phi(x)$ such that $\phi(b)$ realizes $b \circledast \mathrm{B}^{*}$; thus $\Phi_{1}^{2}(m, b, \phi(b))$ realizes the formula which was realized by $\Phi_{1}^{2}(m, b, l)$. Furthermore, $\Phi_{1}^{2}(m, b, \phi(b))$ must be of the form $E_{5}(p, t, c, u, g)$, where $t$ realizes $\mathrm{T}(\boldsymbol{d}, \boldsymbol{b}, \boldsymbol{p})$, where $u$ realizes $\mathrm{U}(\boldsymbol{p}, \boldsymbol{c})$, and where $g$ realizes $c ® C^{*}$. By the induction hypothesis $c$ realizes $C^{*}$ since $g$ realizes $c \circledast C^{*}$. But if $t$ realizes $\mathrm{T}(\boldsymbol{d}, \boldsymbol{b}, \boldsymbol{p})$ and $u$ realizes $\mathrm{U}(\boldsymbol{p}, \mathrm{c})$, then by (1) both $T_{1}(d, b, p)$ and $U(p)=c$ must be true; thus $T_{1}(d, b, p)$ and $U(p)$ realizes $\mathrm{C}^{*}$. Since, for every $b$ which realizes $\mathrm{B}^{*}$, there exists a $p$ such that this last relation holds, we have demonstrated that $d$ realizes $\mathrm{B}^{*} \supset \mathrm{C}^{*}$.

For the second part of the case, let us suppose, recalling the induction hypothesis, that if $\mathrm{C}^{*}$ is realized by $c$, then $c \AA^{*}$ is realized by $\phi(c)$. Now, suppose that $d$ realizes $\mathrm{B}^{*} \supset \mathrm{C}^{*}$ and that $g$ realizes $b \circledast \mathrm{B}^{*}$. Then by the induction hypothesis, $b$ realizes $\mathrm{B}^{*}$. Furthermore, if $\mu w T_{1}(d, b, w)=p$ and $U(p)=c$, 
then $T_{1}(d, b, p)$ holds and $c$ realizes $C^{*}$. Thus $\tau(d, b, p)$ realizes $\mathrm{T}(\boldsymbol{d}, \boldsymbol{b}, \boldsymbol{p})$, $v(p, c)$ realizes $\mathrm{U}(\boldsymbol{p}, \boldsymbol{c})$, and, by the induction hypothesis, $\phi(U(p))$ realizes $c ® C^{*}$. Hence $\exists z\left(T(d, b, z) \& \exists v\left(U(z, v) \& v\left(B C^{*}\right)\right)\right.$ is realized by $E_{5}(p, \tau(d, b, p), U(p), v(p, U(p)), \phi(U(p)))$. Let $f$ be the number which defines the function $\lambda d b g U_{2}^{2}\left(g, E_{5}(p, \tau(d, b, p), U(p), v(p, U(p)), \phi(U(p)))\right)$, where $p$ abbrevates $\mu w T_{1}(d, b, w)$; then $\Phi_{2}\left(S_{2}^{1}(f, d), b, g\right)$ realizes $\exists z(\mathrm{~T}(d, b, z)$ $\left.\& \operatorname{Hv}\left(\mathrm{U}(\mathrm{z}, \mathrm{v}) \& \mathrm{v} \circledast \mathrm{C}^{*}\right)\right)$. We now apply Lemma 4 to show that if $d$ realizes $\mathrm{B}^{*} \supset \mathrm{C}^{*}$, then $E_{2}\left(d, S_{2}\left(S_{2}^{1}(f, d)\right)\right)$ realizes $\exists \mathrm{x} \forall \mathrm{y}\left(\mathrm{y} \circledast \mathrm{B}^{*} \supset \mathrm{Hz}(\mathrm{T}(\mathrm{x}, \mathrm{y}, \mathrm{z})\right.$ $\left.\left.\& \operatorname{Hv}\left(\mathrm{U}(\mathrm{z}, \mathrm{v}) \& \mathrm{v} \circledast \mathrm{C}^{*}\right)\right)\right)$.

(v). Since the formula $b \circledast 7 B^{*}$ is the same as the formula $b \circledast\left(B^{*} \supset 1=0\right)$ and $b$ realizes $7 \mathrm{~B}^{*}$ if and only if $b$ realizes $\mathrm{B}^{*} \supset 1=0$, this case is a particular example of the preceding one.

(vi) Let $\mathrm{A}$ be of the form $\mathrm{Gx} \mathrm{C}(\mathrm{x})$. For the first part of the case, let us assume that $m$ realizes $\mathrm{c} \circledast(\mathrm{GxC}(\mathrm{x}))^{*}$, which has the form $\mathrm{By} \mathrm{Gz}(\mathrm{E}(\mathrm{y}, z, \mathrm{c}) \&$ $\left.z \circledast(C(y))^{*}\right)$. The number $m$ is of the form $E_{4}(n, p, \epsilon(n, p, c), q)$. Since $\epsilon(n, p, c)$ realizes $\mathrm{E}(n, p, c)$, we have $c=2^{n} \cdot 3^{p}$; and by the induction hypothesis, since $q$ realizes $p \circledast(C(n))^{*}, p$ realizes $(C(n))^{*}$; and $c$ realizes $\exists x(C(x))^{*}$.

For the second part of this case, suppose that $c$ realizes $\mathrm{Gx}(\mathrm{C}(\mathrm{x}))^{*}$; then $c$ is of the form $2^{n} \cdot 3^{p}$ and $p$ realizes $(C(n))^{*}$. The induction hypothesis assures that there is a partial recursive function $\lambda x \phi(x)$ such that if $p$ realizes $(\mathrm{C}(n))^{*}$, then $\phi(p)$ realizes $p \circledast(\mathrm{C}(n))^{*}$. Thus if $c$ realizes $\exists \mathrm{x}(\mathrm{C}(\mathrm{x}))^{*}$, the formula $\operatorname{Gw} \operatorname{Hy} \mathrm{z}\left(\mathrm{E}(\mathrm{y}, \mathrm{z}, \mathrm{w}) \& \mathrm{z} 囚(\mathrm{C}(\mathrm{y}))^{*}\right)$ is realized by $E_{5}\left(c,(c)_{0},(c)_{1}\right.$, $\left.\epsilon\left((c)_{0},(c)_{1}, c\right), \phi\left((c)_{1}\right)\right)$.

(vii) Let $\mathrm{A}$ be of the form $\mathrm{VxC}(\mathrm{x})$. For the first part of the case, assume that $m$ realizes $\mathrm{c} \circledast \forall \mathrm{x}(\mathrm{C}(\mathrm{x}))^{*}$, then $m$ realizes $\forall \mathrm{x} \exists \mathrm{y}(\mathrm{T}(\mathrm{c}, \mathrm{x}, \mathrm{y}) \& \mathrm{H} \mathrm{v}(\mathrm{U}(\mathrm{y}, \mathrm{v})$ $\left.\& \mathrm{v}\left((\mathrm{C}(x))^{*}\right)\right)$. Then for every $n$, the number $\Phi_{1}(m, n)$ has the form $E_{5}\left(n_{1}, n_{2}, n_{3}, n_{4}, n_{5}\right)$ where $n_{2}$ realizes $\mathrm{T}\left(\mathrm{c}, \boldsymbol{n}, \boldsymbol{n}_{1}\right)$, where $n_{4}$ realizes $\mathrm{U}\left(\boldsymbol{n}_{1}, \boldsymbol{n}_{3}\right)$, and $n_{5}$ realizes $n_{3} @(\mathrm{C}(n))^{*}$. Thus by (1) we have $T_{1}\left(c, n, n_{1}\right)$ and $U\left(n_{1}\right)=n_{3}$, and by the induction hypothesis $U\left(n_{1}\right)$ realizes $(\mathrm{C}(n))^{*}$. Since this is the case for every $n$, the number $c$ defines a general recursive function and realizes $\mathrm{Vx}(\mathrm{C}(\mathrm{x}))^{*}$.

For the second part of the case, let us assume from the induction hypothesis that if $h$ realizes $(\mathrm{C}(\boldsymbol{n}))^{*}$, then $\phi(h)$ realizes $h \circledast(\mathrm{C}(\boldsymbol{n}))^{*}$. Now suppose that $c$ realizes $\forall x(C(x))^{*}$. If $p=\mu w T_{1}(c, n, w)$ and $U(p)=h$, then $T_{1}(c, n, p)$ and $h$ realizes $(\mathrm{C}(\boldsymbol{n}))^{*}$. Furthermore, $\tau(c, n, p)$ realizes $\mathrm{T}(\mathbf{c}, \boldsymbol{n}, \boldsymbol{p}), v(p, U(p))$ realizes $U(\boldsymbol{p}, \boldsymbol{h})$, and $\phi(\mathrm{U}(\mathrm{p}))$ realizes $\boldsymbol{h}(\mathrm{C}(\boldsymbol{n}))^{*}$. Hence, the formula $\operatorname{\exists y}\left(\mathrm{T}(\mathrm{c}, n, \mathrm{y}) \& \mathrm{Gv}\left(\mathrm{U}(\mathrm{y}, \mathrm{v}) \& \mathrm{v} \circledast(\mathrm{C}(\boldsymbol{n}))^{*}\right)\right)$ is realized by $E_{\mathbf{5}}(p, \tau(c, n, p)$, $U(p), v(p, U(p)), \phi(U(p)))$. Let $f$ define the function $\lambda c n E_{5}(p, \tau(c, n, p), U(p)$, $v(p, U(p)), \phi(U(p)))$, where $p$ abbreviates $\mu w T_{1}(c, n, w)$. Then $\Phi_{1}\left(S_{1}^{1}(f, c), n\right)$ $=\Phi_{2}(f, c, n)$. Now we apply Lemma 4 and obtain finally the result that $E_{5}\left(c, S_{1}\left(S_{1}^{1}(f, c)\right)\right)$ realizes the formula

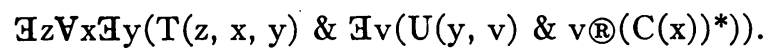


Now we have shown that if $m$ realizes $a ® \mathrm{~A}^{*}$, then $\mathrm{A}^{*}$ is realized by $a$. To obtain the first part of the theorem, let us assume that the order of occurrence of free variables in the formula $\mathrm{a} \circledast \mathrm{A}$ is $\mathrm{a}_{1}, \cdots, \mathrm{a}_{j}, \mathrm{a}_{1}, \mathrm{a}_{j+1}, \cdots, \mathrm{a}_{k}$. Then applying the corollary to Lemma 4 to the function $\lambda n_{1} \cdots n_{j} a n_{j+1} \cdots n_{k} m U_{1}^{1}(a)$, we obtain the result that $a ® A \supset A$ is realizable.

Also we have shown that for each $\mathrm{A}$ there exists some partial recursive function $\lambda x \rho(x)$, such that if $a$ realizes $\mathrm{A}^{*}$, the number $E_{2}(a, \rho(a))$ realizes $\exists \mathrm{x}\left(\mathrm{x} \circledast \mathrm{A}^{*}\right)$. Again we apply the corollary to Lemma 4, this time to the function $\lambda n_{1} \cdots n_{k} a E_{2}(a, \rho(a))$, and obtain the second part of the theorem.

COROLlaRy. Any formula of the form $\mathrm{A} \supset \mathrm{Bx}(\mathrm{x} @ \mathrm{~A})$ or of the form $\mathrm{\exists x}(\mathrm{x} \circledast \mathrm{A})$ $\supset \mathrm{A}$ is realizable.

From the theorem we have the realizability of $a ® A \supset A$. Since the free variable a does not occur in $A$, the formula $\exists x(x \otimes A) \supset A$ is deducible by an application of Rule 12 from a $₫ \mathrm{~A} \supset \mathrm{A}$; and the desired result is obtained from Theorem 1.

\section{PART II}

To consider additional problems concerning the formulas a $₫ A$, which represent the realizability predicate, we must formalize a portion of the theory of recursive functions.

Péter has observed that every primitive recursive function may be defined by recursion equations containing at most two variables and substitution schemata [16, pp. 619-620]. In the present part of the work we shall take this observation as a point of departure to establish a normal form for equations defining primitive recursive functions of two variables. We shall show, then, that in a class of formal systems of recursive number theory primitive recursive functions including course-of-value recursions may be expressed in terms of these normal recursive functions.

In the following work, the formal expression $\mathrm{A} \equiv \mathrm{B}$ will be understood as an abbreviation for the formula $A \supset B \& B \supset A$.

3. Intuitionistic systems of recursive number theory. For the remaining work, intuitionistic systems of recursive number theory, $\mathfrak{S}$, are characterized as follows: a system $\subseteq$ contains in addition to the terms of $S_{1}$, terms of the form $\mathrm{f}\left(\mathrm{t}_{1}, \cdots, \mathrm{t}_{k}\right)$, where $\mathrm{t}_{1}, \cdots, \mathrm{t}_{k}$ are terms and $\mathrm{f}$ is a function symbol or functor; and in addition to the axioms of $S_{1}$, the schema for equality 23; and for each functor $f$ of $S$, a set of axioms of the form specified by (a), (b), or (c) below, which are said to define $f$. The symbol $f$ does not occur in any defining equation preceding its own in the list of axioms. We say that a functor $f$ depends on a functor $g$, in case $g$ appears in the defining equations of $f$, or in case there exists an $h$ which depends on $g$ and $f$ depends on $h$.

We shall agree in the following work to use the same functor in different systems only if the defining equations are the same; thus a system $\mathfrak{S}$ con- 
taining all the theorems of a number of systems will be obtained by choosing as axioms for $\mathfrak{S}$ the set-theoretic sum of the axioms of all the systems.

Of the various forms of equations which describe primitive recursive functions, we shall consider three types.

(a) A functor $\mathrm{f}$ is said to be normal recursive in case it is one of $\nu_{1}, \nu_{2}, \nu_{3}$, or $\nu_{4}$ which have defining equations

$$
\begin{aligned}
& \nu_{1}(\mathrm{a}, \mathrm{b})=0 \text { (the constant function), } \\
& \nu_{2}(\mathrm{a}, \mathrm{b})=\mathrm{a}^{\prime} \text { (the successor function), } \\
& \nu_{3}(\mathrm{a}, \mathrm{b})=\mathrm{a} \text { and } \\
& \nu_{4}(\mathrm{a}, \mathrm{b})=\mathrm{b} \text { (the identity functions), }
\end{aligned}
$$

or if its defining equations are of the form (1) or $(2)$ where $f_{1}, f_{2}, f_{3}$ are normal functors defined by axioms preceding the ones for $f$ :

$$
\begin{aligned}
f(a, b) & =f_{3}\left(f_{1}(a, b), f_{2}(a, b)\right), \\
f(0, b) & =f_{1}(0, b), \\
f\left(a^{\prime}, b\right) & =f_{3}\left(f(a, b), f_{2}(a, b)\right) .
\end{aligned}
$$

(b) A function $f$ is said to be primitive recursive, or primitive recursive in sense (b), if it is one of those appearing in (i)-(iii):

$$
\mathrm{S}(\mathrm{a})=\mathrm{a}^{\prime} \text {, }
$$

$$
0(a)=0 \text {, }
$$

$$
\mathrm{U}_{i}^{n}\left(\mathrm{a}_{1}, \cdots, \mathrm{a}_{n}\right)=\mathrm{a}_{i}
$$

(for positive natural numbers $i$ and $n, i \leqq n$ ),

or if its defining equations are of the form of (1) or (2) where the $f_{i}$ are primitive recursive functors defined by preceding axioms, where $\mathfrak{a}$ abbreviates $\mathrm{a}_{1}, \cdots, \mathrm{a}_{k}$ :

$$
\begin{aligned}
f(\mathfrak{a}) & =\mathrm{f}_{1}\left(\mathrm{f}_{2}(\mathfrak{a}), \cdots, \mathrm{f}_{n}(\mathfrak{a})\right), \\
f(0, \mathfrak{a}) & =\mathrm{f}_{1}(\mathfrak{a}), \\
f\left(\mathfrak{a}^{\prime}, \mathfrak{a}\right) & =\mathrm{f}_{2}(\mathfrak{f}(\mathfrak{a}, \mathfrak{a}), \mathfrak{a}, \mathfrak{a}),
\end{aligned}
$$

or, for a numeral $n$,

$$
\begin{aligned}
f(0) & =n, \\
f\left(a^{\prime}\right) & =f_{2}(f(a), a) .
\end{aligned}
$$

(c) A functor $f$ is said to be a course-of-values functor $[16, p .620]$ if its defining equations are of the form

$$
\begin{aligned}
f(0, \mathfrak{a}) & =f_{1}(\mathfrak{a}), \\
f\left(a^{\prime}, \mathfrak{a}\right) & =f_{2}\left(f\left(t_{1}, \mathfrak{a}\right), \cdots, f\left(t_{n}, \mathfrak{a}\right), a, \mathfrak{a}\right),
\end{aligned}
$$

or of the form (b) (1), where the $f_{i}$ are functors of type (b) or (c), and the system $\subseteq$ in which $\mathrm{f}$ appears contains a formula $\mathrm{a}<\mathrm{b}$ such that 8.2 through 
8.5 of Lemma 10 below are provable, and $t_{i}$ is a term of $\subseteq$ containing only the variables $a, \mathfrak{a}$ such that the formulas

$$
t_{i}<a^{\prime},
$$$$
i=1, \cdots, n \text {, }
$$

are provable in $\mathfrak{S}$.

For the statement of several lemmas concerning the relations among the three types of functors, we introduce the notion of representation of a term or functor by another functor in a system $\mathfrak{S}$.

(1) A functor $f$ of $n$ variables and of type (a), (b), or (c) is represented by a functor $g$ of type (b) in a system $\subseteq$ in case the formula

$$
f\left(a_{1}, \cdots, a_{n}\right)=g\left(a_{1}, \cdots, a_{n}\right)
$$

is provable in $\mathfrak{S}$.

(2) For representation of a functor $f$ of $n$ variables and of type (a), (b), or (c) by a functor $\mathrm{g}$ of type (a), we must distinguish cases (i), (ii), and (iii) for $n=1, n=2$, and $n>2$ respectively. The normal functor $g$ represents the functor $\mathrm{f}$ in $\mathfrak{S}$ in case

$$
\begin{array}{r}
f\left(a_{1}\right)=g\left(a_{1}, a_{2}\right), \\
f\left(a_{1}, a_{2}\right)=g\left(a_{1}, a_{2}\right),
\end{array}
$$

or

$$
f\left(a_{1}, a_{2}, \cdots, a_{n}\right)=g\left(a_{1},\left\{a_{2}, \cdots, a_{n}\right\}\right)
$$

is provable in $\mathfrak{S}$, where $\left\{a_{2}, \cdots, a_{n}\right\}$ is a term which represents a product of powers of successive primes with respective exponents represented by $a_{2}, \cdots, a_{n}$, cf. $\$ 5$ below.

It will also be convenient to refer to the representation of a general term by a functor. Let the free variables in order of appearance in a term $t$ be exactly $a_{1}, \cdots, a_{n}$, then, if in (1) and (2) above, we replace $f\left(a_{1}, \cdots, a_{n}\right)$, $f\left(a_{1}\right), f\left(a_{1}, a_{2}\right)$ by $t$, we obtain a definition for the representation of a term $t$ by a functor $g$ of type (b) or (a) in a formal system $\mathbf{S}$.

If $t$ is represented by a functor $g$ of type (a) or (b), then there also exists a functor $g^{\prime}$ of the same type as $g$ which represents the term $U_{k+1}^{k+1}\left(a_{v_{1}}, \cdots, a_{v_{k}}, t\right)$, where $k \geqq n$ and $\mathrm{a}_{\boldsymbol{v}_{1}}, \cdots, \mathrm{a}_{\boldsymbol{v}_{k}}$ is any permutation of $\mathrm{a}_{1}, \cdots, \mathrm{a}_{n}$ and possibly additional variables. Then $\mathrm{g}^{\prime}\left(\mathrm{a}_{\boldsymbol{n}_{1}}, \cdots, \mathrm{a}_{v_{k}}\right)=\mathrm{t}$ is provable in the system $\mathfrak{S}$ of the latter representation.

LEMMA 7. Every term formed by composition from terms containing no functors except type (b) functors is represented by a type (b) functor in some S.

The demonstration is a trivial one, effected by the addition of axioms of Schema (b) (1) which are constructed by successive application of the functors $U_{i}^{n}$. 
By this lemma and the remark immediately preceding, if in (b) (1) the right member is replaced by any term containing no other than type (b) functors and no other than the variables shown, or if in (b) (2), $f_{1}(\mathfrak{a})$ and $f_{2}(c, a, \mathfrak{a})$ (or for the second case, $f_{2}(c, a)$ ) are so replaced, the resulting formula or pair of formulas is provable for some type (b) functor $f$ in an appropriate system S.

LEMMA 8. If every functor appearing in a term $\mathrm{t}$ is a functor of one or of two variables which is represented by a normal functor, and if $t$ contains only one or two variables, then $\mathrm{t}$ is represented by a normal functor in some system $\mathfrak{S}$.

The demonstration is again a trivial one, effected this time by addition of axioms of Schema (a) (1) which are constructed by successive applications of the normal identity functors $\nu_{3}$ and $\nu_{4}$ and the representing functors whose existence is guaranteed by the hypothesis of the lemma.

4. An equivalence between any system $\subseteq$ and $S_{1}$. Gödel has shown that for every formula A of a system of classical number theory, there exists a corresponding formula $\mathrm{A}^{\prime}$ of a system of intuitionistic number theory, such that if $\mathrm{A}$ is provable in the former, then $\mathrm{A}^{\prime}$ is provable in the latter [6]. This result, however, is for the intuitionistic formalization of arithmetic of Heyting [9], from which ours differs principally in the introduction of numerals and the absence of a formalization of the substitution operation. Nevertheless, it is easily seen that Gödel's treatment affords a demonstration of an equivalence between any $\mathfrak{S}$ and a classical system $\mathbb{\mathfrak { S }}$ which differs from $\mathfrak{S}$ only in having Schema 8 replaced by the schema 77A $\supset$ A. Under this correspondence of $\mathbb{S}$ to $\subseteq$, we shall let $C_{1}$ correspond to $S_{1}$, and so on.

We define the formula $A^{\prime}$ in the following manner: (1) If $A$ is an elementary formula, $\mathrm{A}^{\prime}$ is $\mathrm{A}$. (2) For every $\mathrm{A}$ of one of the forms: $7 \mathrm{~B}, \mathrm{~B} \& \mathrm{C}$, $\mathrm{B} \supset \mathrm{C}, \mathrm{B} \vee \mathrm{C}, \mathrm{Vx} \mathrm{B}(\mathrm{x})$, 马x $\mathrm{B}(\mathrm{x}) ; \mathrm{A}^{\prime}$ is defined as: $7 \mathrm{~B}^{\prime}, \mathrm{B}^{\prime} \& \mathrm{C}^{\prime}, 7\left(\mathrm{~B}^{\prime} \& 7 \mathrm{C}^{\prime}\right)$, $7\left(>B^{\prime} \& 7 C^{\prime}\right), \forall x B^{\prime}(x), 7 \forall x>B^{\prime}(x)$, where $(B(a))^{\prime}$ is written $B^{\prime}(a)$ and $\mathrm{B}^{\prime}(\mathrm{x})$ is the result of substituting a variable $\mathrm{x}$ not occurring in $\mathrm{B}(\mathrm{a})$ for a in $B^{\prime}(a)$.

Lemma 9. If $\mathrm{A}$ is provable in any classical system $\mathfrak{E}$, then $\mathrm{A}^{\prime}$ is provable in the corresponding intuitionistic system $\mathbb{S}$.

We observe first that the formula

$$
a=b \vee 7 a=b
$$

is provable in $S_{1}$ and thus is provable in every $\mathbb{S}$. Then the demonstration may be completed by the method of Gödel.

COROLlARY 9.1. For every primitive recursive predicate $R\left(a_{1}, \cdots, a_{k}\right)$ of exactly the variables exhibited, there exists a formula $\mathrm{R}\left(\mathrm{a}_{1}, \cdots, \mathrm{a}_{k}\right)$ containing exactly the free variables exhibited, and such that, for natural numbers $n_{1}, \cdots, n_{k}$, if $R\left(n_{1}, \cdots, n_{k}\right)$, then $\mathrm{R}\left(n_{1}, \cdots, n_{k}\right)$ is provable in $S_{1}$, and if $\bar{R}\left(n_{1}, \cdots, n_{k}\right)$, 
then $7 \mathrm{R}\left(\boldsymbol{n}_{1}, \cdots, \boldsymbol{n}_{k}\right)$ is provable in $S_{1}$. Furthermore, there exists a partial recursive function $\lambda x_{1} \cdots x_{k} \rho\left(x_{1}, \cdots, x_{k}\right)$ such that $\rho\left(n_{1}, \cdots, n_{k}\right)$ realizes $\mathrm{R}\left(\boldsymbol{n}_{1}, \cdots, \boldsymbol{n}_{k}\right)$ if that formula is realizable.

For every primitive recursive predicate $R\left(x_{1}, \cdots, x_{k}\right)$ there exists a system of classical number theory $\mathbb{S}$ containing a functor $\mathrm{f}$ such that according as $R\left(n_{1}, \cdots, n_{k}\right)$ or $\bar{R}\left(n_{1}, \cdots, n_{k}\right)$, the formula $\mathrm{f}\left(n_{1}, \cdots, n_{k}\right)=0$ or the formula $7 \mathrm{f}\left(\boldsymbol{n}_{1}, \cdots, \boldsymbol{n}_{k}\right)=0$ is provable in $\mathfrak{C}$. Furthermore, by the HilbertBernays result on the elimination of the $\iota$-symbol [10, vol. 1, pp. $457 \mathrm{ff}$.], there exists a formula $\mathrm{R}\left(\mathrm{a}_{1}, \cdots, \mathrm{a}_{k}\right)$ of $C_{1}$ containing just the free variables indicated, and such that according as $R\left(n_{1}, \cdots, n_{k}\right)$ or $\bar{R}\left(n_{1}, \cdots, n_{k}\right)$, either $\mathrm{R}\left(\boldsymbol{n}_{1}, \cdots, \boldsymbol{n}_{k}\right)$ or $7 \mathrm{R}\left(\boldsymbol{n}_{1}, \cdots, \boldsymbol{n}_{k}\right)$ is provable in $C_{1}$; thus either $\mathrm{R}^{\prime}\left(\boldsymbol{n}_{\mathbf{1}}, \cdots, \boldsymbol{n}_{k}\right)$ or $\left(7 \mathrm{R}\left(\boldsymbol{n}_{1}, \cdots, \boldsymbol{n}_{k}\right)\right)^{\prime}$ is provable in $S_{\mathbf{1}}$. Noting that $7 \mathrm{R}^{\prime}\left(\boldsymbol{n}_{1}, \cdots, \boldsymbol{n}_{k}\right)$ is the same formula as $\left(7 \mathrm{R}\left(\boldsymbol{n}_{1}, \cdots, \boldsymbol{n}_{k}\right)\right)^{\prime}$, we have the first part of the corollary with $\mathrm{R}^{\prime}\left(\mathrm{a}_{1}, \cdots, \mathrm{a}_{k}\right)$ chosen for the formula $\mathrm{R}\left(\mathrm{a}_{1}, \cdots, \mathrm{a}_{k}\right)$ mentioned in the statement of the corollary.

In addition we note that the formula $f\left(a_{1}, \cdots, a_{k}\right)=0 \equiv R\left(a_{1}, \cdots, a_{k}\right)$ is provable in $\mathfrak{E}$, and thus $\left(\mathrm{f}\left(\mathrm{a}_{1}, \cdots, \mathrm{a}_{k}\right)=0 \equiv \mathrm{R}\left(\mathrm{a}_{1}, \cdots, \mathrm{a}_{k}\right)\right)^{\prime}$ is provable in $\mathfrak{S}$. Applying Hilfssatz 2 of Gödel's work, we obtain the provability in $\mathfrak{S}$ of $\mathrm{f}\left(\mathrm{a}_{1}, \cdots, \mathrm{a}_{k}\right)=0 \equiv \mathrm{R}^{\prime}\left(\mathrm{a}_{1}, \cdots, \mathrm{a}_{k}\right)$. From Corollary $1.1, \mathrm{f}\left(\mathrm{a}_{1}, \cdots, \mathrm{a}_{k}\right)=0$ $\equiv \mathrm{R}^{\prime}\left(\mathrm{a}_{1}, \cdots, \mathrm{a}_{k}\right)$ is realizable, suppose by the number $r$. Then $\mathrm{R}^{\prime}\left(\boldsymbol{n}_{1}, \cdots, \boldsymbol{n}_{k}\right)$ is realizable if and only if $\mathrm{f}\left(\boldsymbol{n}_{1}, \cdots, \boldsymbol{n}_{k}\right)=0$ is realizable. By Lemma 3 , if $\mathrm{R}^{\prime}\left(n_{1}, \cdots, n_{k}\right)$ is realizable, it is realized by $\Phi_{1}^{k+1}\left((r)_{0}, n_{1}, \cdots, n_{k}, 0\right)$. Thus the partial recursive function $\lambda x_{1} \cdots x_{k} \Phi_{1}^{k+1}\left((r)_{0}, x_{1}, \cdots, x_{k}, 0\right)$ may be chosen for $\lambda x_{1} \cdots x_{k} \rho\left(x_{1}, \cdots, x_{k}\right)$.

COROLlARY 9.2. If $\mathrm{D}$ is a formula which contains no quantifiers and $\mathrm{D}$ is provable in any classical system $\mathfrak{}$, then $\mathrm{D}$ is also provable in the corresponding intuitionistic system $\mathfrak{S}$.

First, for a formula $\mathrm{D}$ containing no quantifiers, it may be shown by induction on the number of logical symbols in $D$ that $D \vee>D$ is provable in $\mathfrak{S}$. Using this result, again by induction, we may show that for every $\mathrm{D}$ containing no quantifiers the formula $\mathrm{D}^{\prime} \equiv \mathrm{D}$ is provable in $\subseteq$. These two demonstrations are straightforward and follow easily from familiar rules for the intuitionistic predicate calculus $\left({ }^{8}\right)$. Application of the lemma completes the demonstration.

5. The system $S_{2}$. Now, we shall consider a fundamental system of intuitionistic number theory $S_{2}$, which will contain additional functors to those of $S_{1}$. To avoid tedious detail in construction of formal proofs for fundamental formulas of number theory, we utilize the work of Hilbert-Bernays [10, vol. 1,

( 9$)[8,4]$. The reader is referred to these papers for a discussion of the intuitionistic predicate calculus. Details concerning the provability of certain formulas will be required for the following work. 
pp. 286-382] in a formal system of classical number theory and apply Corollary 9.2 to obtain the provability of the formulas in an intuitionistic system.

The system $S_{2}$ has the following groups of axioms:

(1) The axioms of $S_{1}$.

(2) Axioms of the form of Schema 23.

(3) Axioms which define the functors of type (b) which appear in the formulas 1.0 through 10.11 in Lemma 10 and axioms for the additional functors upon which these functors depend.

(4) Axioms for normal functors which represent the functors of type (b) appearing in formulas 1.0 through 10.11 and axioms for functors upon which these normal functors depend (axioms for $\nu_{1}, \cdots, \nu_{4}$ included).

In Lemma 10 we shall collect for convenient reference the fundamental number-theoretic formulas required for later work.

In the system $S_{2}$, the term abbreviated $\mathrm{a}^{\mathrm{b}}$ corresponds to the intuitive function $a^{b}$. The formula $7 \delta(b, a)=0$, abbreviated $a<b$, corresponds to the intuitive relation $a<b$. The term $\pi(a)$ corresponds to the prime number function; the formula $\pi(n)=k$ is provable when $k$ is the $(n+1)$ th prime number. The term abbreviated $(a)_{b}$ corresponds to the function $(a)_{b}$, cf. $\$ 1$, list of recursive functions $(\mathrm{g})$; the formula $(m)_{n}=k$ is provable when $k$ is the exponent of the $(n+1)$ th prime number in a factorization of $m$ into a product of powers of distinct primes. The expression $\left\{a_{0}, \cdots, a_{n}\right\}$ is used to abbreviate the term $\pi(0)^{a_{0}} \ldots \cdots \pi(n)^{a_{n}}$.

Lemma 10. There exists a formal system $S_{2}$ having the properties just described and in which formulas 1.0 through 10.11 (below) are provable and in which each of the functors appearing in these formulas is represented by a normal recursive functor.

For each of the formulas 1.0 through 10.11 , either there appears a proof in the classical system of number theory of Hilbert-Bernays or else a proof may easily be constructed for the formula from the results found there. (The reader should note differences in notation for certain of the functions mentioned in the paragraph preceding Lemma 10.) Corollary 9.2 assures the existence of a formal system of intuitionistic number theory in which the formulas 1.0 through 10.10 are provable. Also Lemma 7 and the accompanying remarks are involved. The provability of 10.11 in a suitable intuitionistic system follows from Corollary 11.1 below.

The fact that each of the functors that appears in these formulas is represented by a normal functor is easily verified by direct construction for the functors in 1.1-9.9. The remaining functors may be defined by additional recursion equations including ones of the following forms:

$$
\sum_{x=0}^{0} f(x)=f(0), \quad \sum_{x=0}^{a^{\prime}} f(x)=\left(\sum_{x=0}^{a} f(x)\right)+f\left(a^{\prime}\right) .
$$


(ii)

$$
\prod_{x=0}^{0} f(x)=f(0), \quad \prod_{x=0}^{a^{\prime}} f(x)=\left(\prod_{x=0}^{a} f(x)\right) \cdot f\left(a^{\prime}\right)
$$

That such functors may be represented by normal functors in a suitable system may easily be shown by an induction proof.

1.0. $a=b \vee 7 a=b$.

1.1. $a+(b+c)=(a+b)+c$.

1.2. $a+b=b+a$.

1.3. $a \cdot(b \cdot c)=(a \cdot b) \cdot c$.

1.4. $a \cdot b=b \cdot a$.

1.5. $a \cdot(b+c)=(a \cdot b)+(a \cdot c)$.

1.6. $1 \cdot a=a$.

1.7. $a \cdot b=0 \equiv a=0 \vee b=0$.

1.8. $a+b=0 \equiv a=0 \& b=0$.

2.1. $\alpha(0)=0$.

2.2. $\alpha\left(a^{\prime}\right)=1$.

2.3. $\alpha(a)=0 \equiv a=0$.

3.1. $\beta(0)=1$.

3.2. $\beta\left(\mathrm{a}^{\prime}\right)=0$.

3.3. $\beta(a)=0 \equiv 7 a=0$.

3.4. $\beta(a) \cdot b=0 \equiv(a=0 \supset b=0)$.

4.1. $\delta(0)=0$.

4.2. $\delta\left(a^{\prime}\right)=a$.

5.1. $\delta(b, 0)=b$.

5.2. $\delta\left(b, a^{\prime}\right)=\delta(\delta(b, a))$.

6.1. $\epsilon(a, b)=\delta(a, b)+\delta(b, a)$.

6.2. $\epsilon(a, b)=0 \equiv a=b$.

7.1. $b^{0}=1$.

7.2. $\mathrm{b}^{\mathrm{a}^{\prime}}=\mathrm{b}^{\mathrm{a}} \cdot \mathrm{b}$.

8.1. $a<b \equiv 7 \delta(b, a)=0$.

8.2. $7 \mathrm{a}<\mathrm{a}$.

8.3. $a<a^{\prime}$.

8.4. $a<b \supset a^{\prime}=b \vee a^{\prime}<b$.

8.5. $a<b \& b<c \supset a<c$.

8.6. $a<b \vee b<a \vee a=b$.

8.7. $7 a<0$.

8.8. $a<b \supset 7 a=b$.

8.9. $a^{\prime}<b \supset a<b$.

8.10. $a<b \equiv a+c<b+c$.

8.11. $7 c=0 \supset(a<b \equiv c \cdot a<c \cdot b)$.

9.1. $a \leqq b \equiv a<b \vee a=b$.

9.2. $a \leqq 0 \supset a=0$.

9.3. $a \leqq b \supset a \leqq b^{\prime}$. 
9.4. $a \leqq b^{\prime} \equiv a \leqq b \vee a=b^{\prime}$.

9.5. $\mathrm{a} \leqq \mathrm{c} \supset 7 \mathrm{c}^{\prime}=\mathrm{a}$.

9.6. $a \leqq b \equiv a<b^{\prime}$.

9.7. $a<b \equiv 7 b \leqq a$.

9.8. $a^{\prime} \leqq b \supset a \leqq b$.

9.9. $0<\mathrm{a}^{\prime}$.

10.1. $(0)_{\mathrm{a}}=0$.

10.2. $(1)_{a}=0$.

10.3. $\left((\pi(a))^{b}\right)_{a}=b$.

10.4. $\quad 7 \mathrm{a}=\mathrm{b} \supset\left((\pi(\mathrm{a}))^{\mathrm{c}}\right)_{\mathrm{b}}=0$.

10.5. $7 a \cdot b=0 \supset(a \cdot b)_{c}=(a)_{0}+(b)_{c}$.

10.6. $7 \mathrm{a}=0 \supset(\mathrm{a})_{\mathrm{b}}<\mathrm{a}$.

10.7. $k \leqq n \supset\left\{\mathrm{a}_{0}, \cdots, a_{n}\right\}_{k}=\mathrm{a}_{k}$.

10.8. $n<k \supset\left\{a_{0}, \cdots, a_{n}\right\}_{k}=0$.

10.9. $7(\pi(\mathrm{a}))^{\mathrm{b}}=0$.

10.10. $a<c \supset(a)_{c}=0$.

10.11. $a \leqq b \& \forall x\left(x \leqq b \supset(a)_{x}=(b)_{x}\right) \supset a=b$.

6. Formulas of Class $\Gamma$. The formulas of the predicate calculus which contain only bounded quantifiers are known to represent recursive relations ( $[5$; 10 , vol. 1 , pp. $310 \mathrm{ff}$.]); to aid our formalization of intuitionistic number theory we introduce the following definition.

A formula $A$ of a system $\subseteq$ is said to be a formula of Class $\Gamma$ in case:

(1) A is an elementary formula.

(2) $B$ and $C$ are of Class $\Gamma$, and $A$ is $7 B, B \vee C, B \& C$, or $B \supset C$.

(3) $B(a)$ is of Class $\Gamma$ and $A$ is $\forall x(x \leqq b \supset B(x))$, or $\exists x(x \leqq b \& B(x))$. (The symbol $\leqq$ may be replaced by $<$.)

Lemma 11. If $\mathrm{A}\left(\mathrm{a}_{1}, \cdots, \mathrm{a}_{k}\right)$ is a formula of Class $\Gamma$ containing exactly the free variables exhibited, there exists a system $\mathfrak{T}$ containing a functor $\mathrm{f}$ such that in $\mathfrak{T}$ the formula $\mathrm{f}\left(\mathrm{a}_{1}, \cdots, \mathrm{a}_{k}\right)=0 \equiv \mathrm{A}\left(\mathrm{a}_{1}, \cdots, \mathrm{a}_{k}\right)$ is provable.

The proof of this lemma is essentially that outlined in Hilbert-Bernays, vol. 1, pp. 310-315, for the system of classical number theory.

It is proved by induction on the number of logical symbols in $\mathrm{A}\left(\mathrm{a}_{1}, \cdots, \mathrm{a}_{k}\right)$. If this is an elementary formula, then it is of the form $p=q$, where $p$ and $q$ are terms. Formula 6.2 of Lemma 10 gives us the provability of

$$
\epsilon(\mathrm{p}, \mathrm{q})=0 \equiv \mathrm{p}=\mathrm{q} \text {. }
$$

This affords a basis for an induction proof of the lemma.

Assume that the formulas:

$$
\mathrm{t}=0 \equiv \mathrm{B}, \quad \mathrm{s}=0 \equiv \mathrm{C},
$$

are provable in some $\mathfrak{S}$. Then using $3.3,1.7,1.8$ and 3.4 of Lemma 10 , we have the following: 


$$
\begin{aligned}
\beta(t) & =0 \equiv 7 \mathrm{~B}, \\
\mathrm{t} \cdot \mathrm{s} & =0 \equiv \mathrm{B} \vee \mathrm{C}, \\
\mathrm{t}+\mathrm{s}=0 & \equiv \mathrm{B} \& \mathrm{C}, \\
\beta(\mathrm{t}) \cdot \mathrm{s}=0 & \equiv \mathrm{B} \supset \mathrm{C} .
\end{aligned}
$$

For the forms containing bounded quantifiers, assume that the formula

$$
t(a)=0 \equiv A(a)
$$

is provable in some $\mathfrak{S}$. Using 9.4 and rules of the predicate calculus the following are provable:

$$
\begin{aligned}
& \forall x\left(x \leqq a^{\prime} \supset A(x)\right) \equiv \forall x(x \leqq a \supset A(x)) \& A\left(a^{\prime}\right), \\
& H x\left(x \leqq a^{\prime} \& A(x)\right) \equiv A x(x \leqq a \& A(x)) \vee A\left(a^{\prime}\right) .
\end{aligned}
$$

Then, by the defining equations for $\sum_{x-0}^{\mathrm{a}} \mathrm{t}(\mathrm{x})$ and $\prod_{x=0}^{\mathrm{a}} \mathrm{t}(\mathrm{x})$ as given following Lemma 10, and by application of the induction schema 13, using 9.2 to obtain the basis, we have:

$$
\begin{aligned}
& \sum_{x=0}^{a} t(x)=0 \equiv \forall x(x \leqq a \supset A(x)), \\
& \prod_{x=0}^{g} t(x)=0 \equiv \mathcal{H} x(x \leqq a \& A(x)) .
\end{aligned}
$$

For the case concerning the similar formulas which contain $<$, we observe that using formulas from Lemma 10 and the predicate calculus, the following formulas are provable:

$$
\begin{aligned}
& \forall x(x<a \supset A(x)) \equiv a=0 \vee \forall x(x \leqq \delta(a) \supset A(x)), \\
& \forall x(x<a \& A(x)) \equiv 7 a=0 \& B x(x \leqq \delta(a) \& A(x)) .
\end{aligned}
$$

CoRollary 11.1. If a formula A of Class $\Gamma$ is provable in a classical system $\mathfrak{C}$, then there exists an intuitionistic system $\mathfrak{I}$ in which $\mathrm{A}$ is also provable.

By the work of Hilbert-Bernays [10, vol. 1, pp. $310 \mathrm{ff}$.], for suitable choice of $t$, the formula $t=0 \equiv A$ is provable in some classical system $D$, which may be taken to include $\mathfrak{C}$. Let $\mathfrak{T}$ be the intuitionistic system corresponding to $\mathfrak{D}$. By a comparison of the work of Hilbert-Bernays and the proof of Lemma 11, it follows that $t=0 \equiv \mathrm{A}$ is also provable in $\mathfrak{T}$. By Corollary 9.2 , if $t=0$ is provable in $\mathfrak{E}$, it is also provable in $\mathfrak{T}$. If $A$ is provable in $\mathfrak{E}$, hence in $\mathfrak{D}$, then $t=0$ is provable in $\mathfrak{D}$, and hence in $\mathfrak{T}$, and finally $A$ is provable in $\mathfrak{T}$.

CoRollary 11.2. For every formula A of Class $\Gamma$, there exists a system $\mathfrak{T}$ such that in $\mathfrak{T}$ the formula $\mathrm{A} \vee 7 \mathrm{~A}$ is provable.

LEMma 12. For every formula $\mathrm{A}(\mathrm{a})$ of Class $\Gamma$ in $\mathfrak{S}$, there exists $a \mathfrak{T}$ in which 
the formula

$$
A(a) \supset \mathcal{H x}(A(x) \& \forall y(A(y) \supset x \leqq y))
$$

is provable.

This is the least number principle, and the proof given by Hilbert and Bernays may be used in a system containing $S_{2}$ [10, vol. 1 , pp. $283 \mathrm{ff}$.], the only addition required, excepting changes in notation, being the observation that in some system $\mathfrak{T}$ the formula $\exists x(A(x) \& x<a) \vee 7 \exists x(A(x)$ $\& \mathrm{x}<\mathrm{a})$ is provable. This follows immediately from Corollary 11.2.

7. Reduction to normal recursion. We now consider the problem of representing any recursive functor by a normal recursive one.

Lemma 13. For every type (c) functor $\mathrm{f}$ of a system $\mathfrak{S}$, there exists a system $\mathfrak{T}$ containing a type (b) functor $\mathrm{f}^{\prime}$ such that the formula $\mathrm{f}(\mathrm{a}, \mathfrak{a})=\mathrm{f}^{\prime}(\mathrm{a}, \mathfrak{a})$ is provable in $\mathfrak{T}$.

The proof is a formalization of that given by Peter [16, p. 620], and proceeds by induction on the number of applications of Schema (c) which are not applications of Schema (b) (2) in the definition of $f$. The basis is trivial, for if $f$ is defined without any such application of Schema (c), then $f$ is a type (b) functor as well as a type (c) functor.

For the induction step, assume that the axioms defining $f$ are

$$
\begin{aligned}
f(0, \mathfrak{a}) & =\mathrm{g}(\mathfrak{a}), \\
\mathbf{f}\left(\mathbf{a}^{\prime}, \mathfrak{a}\right) & =\mathrm{h}\left(\mathbf{f}\left(\mathrm{t}_{1}(\mathfrak{a}, \mathfrak{a}), \mathfrak{a}\right), \cdots, f\left(t_{n}(\mathfrak{a}, \mathfrak{a}), \mathfrak{a}\right), \mathfrak{a}, \mathfrak{a}\right) ;
\end{aligned}
$$

that for $i=1, \cdots, n, t_{i}(a, a)<a^{\prime}$ is provable in $\mathfrak{T}$; and that there exist type (b) recursive functors $g^{\prime}, h^{\prime}$, and $t_{i}^{\prime}$ such that the formulas $g(a)=g^{\prime}(a)$, $\mathrm{h}\left(\mathrm{c}_{1}, \cdots, \mathrm{c}_{n}, \mathrm{a}, \mathfrak{a}\right)=\mathrm{h}^{\prime}\left(\mathrm{c}_{1}, \cdots, \mathrm{c}_{n}, \mathrm{a}, \mathfrak{a}\right)$, and $\mathrm{t}_{\mathfrak{i}}(\mathrm{a}, \mathfrak{a})=\mathrm{t}_{\mathfrak{i}}^{\prime}(\mathrm{a}, \mathfrak{a})$ are provable in $\mathfrak{S}$.

Then by Lemma 7 there exist type (b) functors $p$, $q$, and $f^{\prime}$ such that in a system obtained from $\subseteq$ by adjunction of the requisite axioms the following formulas are provable:

$$
\begin{aligned}
& \mathrm{p}(\mathrm{a}, \mathfrak{a}, \mathrm{d})=\mathrm{h}^{\prime}\left((\mathrm{d})_{\mathfrak{t}_{1}^{\prime}(\mathrm{a}, \mathfrak{a})}, \cdots,(\mathrm{d})_{\boldsymbol{t}_{n}(\mathrm{a}, \mathfrak{a})}^{\prime}\right. \\
& \mathrm{q}(0, \mathfrak{a})=\pi(0) \mathbf{z}^{\prime}(\mathfrak{a}) . \\
& \left.\mathrm{q}\left(\mathrm{a}^{\prime}, \mathfrak{a}\right)=\mathrm{q}(\mathrm{a}, \mathfrak{a}) \cdot \pi\left(a^{\prime}\right)^{p(a, a, ~}, q(a, a)\right) . \\
& \mathbf{f}^{\prime}(\mathfrak{a}, \mathfrak{a})=(\mathrm{q}(\mathrm{a}, \mathfrak{a}))_{\mathbf{a}} \text {. }
\end{aligned}
$$

We shall now show that the formula

$$
\mathrm{c}<\mathrm{a} \supset(\mathrm{q}(\mathrm{c}, \mathfrak{a}))_{\mathrm{a}}=0
$$

is provable in the enlarged system $\mathfrak{T}^{\prime}$. This is established by application of Schema 13, induction on c, as follows. From (ii), and 8.8 and 10.4 from Lemma 
10 , we have $0<a \supset(q(0, a))_{a}=0$, which is the basis formula for the induction proof. By 8.9, if A abbreviates the formula (v), we have

$$
A \supset\left(c^{\prime}<a \supset(q(c, a))_{a}=0\right) \text {. }
$$

Thence, using (iii), and applying 10.5 (using 10.9, (ii), (iii) and Schema 13 to obtain the premise), $8.8,10.4$, Schema 23 , and the propositional calculus, we have

$$
\text { A } \supset\left(c^{\prime}<a \supset\left(q\left(c^{\prime}, a\right)\right)_{a}=0\right),
$$

which is the formula for the induction step. The application of Schema 13 is now made, completing the demonstration that (v) is provable.

We shall next show, again by application of Schema 13, induction on c, that the formula

$$
\forall x\left(x \leqq c \supset f^{\prime}(x, \mathfrak{a})=(q(c, \mathfrak{a}))_{x} \& f(x, \mathfrak{a})=f^{\prime}(x, \mathfrak{a})\right)
$$

is provable in the enlarged system $\mathfrak{T}^{\prime}$.

From (ii), (iv), and the induction hypothesis of the lemma, and 10.3, it is easily shown by Schema 23 that $f^{\prime}(0, \mathfrak{a})=(\mathfrak{q}(0, \mathfrak{a}))_{0} \& f(0, \mathfrak{a})=f^{\prime}(0, \mathfrak{a})$ is provable. Then from 9.2 , by means of Schema 23 and the predicate calculus, we have the basis formula

$$
\forall x\left(x \leqq 0 \supset f^{\prime}(x, \mathfrak{a})=(q(0, \mathfrak{a}))_{x} \& f(x, \mathfrak{a})=f^{\prime}(x, \mathfrak{a})\right)
$$

for the induction proof of (vi).

To obtain the formula for the induction step, we start by noting from (ii), (iii), (v), 8.3, 10.3, and 10.5 that

$$
\left(\mathrm{q}\left(\mathrm{c}^{\prime}, \mathfrak{a}\right)\right)_{\mathfrak{o}^{\prime}}=\mathrm{p}(\mathrm{c}, \mathfrak{a}, \mathrm{q}(\mathrm{c}, \mathfrak{a}))
$$

is provable. Thence, using (i), 9.6, and Schema 23 , noting that $t_{i}(c, a)<c^{\prime}$ and $t_{i}(a, \mathfrak{a})=t_{\mathfrak{i}}^{\prime}(a, \mathfrak{a})$ are provable, and using $B$ to abbreviate (vi), we have $\mathrm{B} \supset\left(\mathrm{q}\left(\mathrm{c}^{\prime}, \mathfrak{a}\right)\right)_{\mathfrak{o}^{\prime}}=\mathrm{h}^{\prime}\left(\mathrm{f}^{\prime}\left(\mathrm{t}_{1}(\mathrm{c}, \mathfrak{a}), \mathfrak{a}\right), \cdots, \mathrm{f}^{\prime}\left(\mathrm{t}_{n}(\mathbf{c}, \mathfrak{a}), \mathfrak{a}\right), \mathrm{c}, \mathfrak{a}\right) \& \mathrm{f}\left(\mathrm{t}_{1}(\mathrm{c}, \mathfrak{a}), \mathfrak{a}\right)$ $=\mathrm{f}^{\prime}\left(\mathrm{t}_{1}(\mathrm{c}, \mathfrak{a}), \mathfrak{a}\right) \& \cdots \& \mathrm{f}\left(\mathrm{t}_{n}(\mathrm{c}, \mathfrak{a}), \mathfrak{a}\right)=\mathrm{f}^{\prime}\left(\mathrm{t}_{n}(\mathrm{c}, \mathfrak{a}), \mathfrak{a}\right)$. From this, (iv), and the formula for $h^{\prime}$ in the induction hypothesis for the lemma and Schema 23, we have

$$
\text { B } \supset f^{\prime}\left(c^{\prime}, \mathfrak{a}\right)=\left(q\left(c^{\prime}, \mathfrak{a}\right)\right)_{\mathfrak{o}^{\prime}} \& f\left(c^{\prime}, \mathfrak{a}\right)=f^{\prime}\left(c^{\prime}, \mathfrak{a}\right) .
$$

If we apply Schema 23, this gives

$$
\text { B } \supset\left(a=c^{\prime} \supset f^{\prime}(a, \mathfrak{a})=\left(q\left(c^{\prime}, \mathfrak{a}\right)\right)_{a} \& f(a, \mathfrak{a})=f^{\prime}(a, \mathfrak{a})\right) .
$$

If we use 9.5, (ii), (iii), 10.4 and 10.5, the formulaa $\leqq c \supset\left(q\left(c^{\prime}, \mathfrak{a}\right)\right)_{a}=(q(c, a))_{a}$ is provable, and the following is also provable:

$$
\text { B } \supset\left(a \leqq c \supset f^{\prime}(a, a)=\left(q\left(c^{\prime}, \mathfrak{a}\right)\right)_{\mathfrak{a}} \& f(a, \mathfrak{a})=f^{\prime}(a, \mathfrak{a})\right) .
$$

Using 9.4 to combine the last two displayed formulas, and applying Schema 9, we have finally 


$$
B \supset \forall x\left(x \leqq c^{\prime} \supset f^{\prime}(x, \mathfrak{a})=\left(q\left(c^{\prime}, \mathfrak{a}\right)\right)_{x} \& f(x, \mathfrak{a})=f^{\prime}(x, \mathfrak{a})\right),
$$

which is the formula for the induction step for the proof of (vi) by Schema 13. This completes our demonstration that (vi) is provable in $\mathfrak{I}^{\prime}$.

Now $a \leqq a$ is provable, and using this in (vi) we have the provability of $f(a, \mathfrak{a})=f^{\prime}(\mathfrak{a}, \mathfrak{a})$ in $\mathfrak{T}^{\prime}$.

LEMma 14. For every type (b) functor $\mathrm{f}$ of any $\mathfrak{S}$ there exists a $\mathfrak{I}$ containing a normal functor $\mathrm{f}^{\prime}$ such that in $\mathfrak{T}$, either $\mathrm{f}(\mathrm{a})=\mathrm{f}^{\prime}(\mathrm{a}, \mathrm{b}), \mathrm{f}(\mathrm{a}, \mathrm{b})=\mathrm{f}^{\prime}(\mathrm{a}, \mathrm{b})$, or $\mathrm{f}(\mathrm{a}, \mathfrak{a})=\mathrm{f}^{\prime}(\mathrm{a},\{\mathfrak{a}\})$ is provable, according as the functor $\mathrm{f}$ takes 1,2 , or more than 2 arguments.

The lemma is proved by induction on the number of functors upon which $f$ depends.

For the basis, we have a representation for $0(a)$ by $\nu_{1}(a, b)$ and for $S(a)$, by $\nu_{2}(\mathrm{a}, \mathrm{b})$. The terms $\mathrm{U}_{1}^{1}(\mathrm{a})$ and $\mathrm{U}_{1}^{2}(\mathrm{a}, \mathrm{b})$ are both represented by $\nu_{3}(\mathrm{a}, \mathrm{b})$, $\mathrm{U}_{2}^{2}(\mathrm{a}, \mathrm{b})$ is represented by $\nu_{4}(\mathrm{a}, \mathrm{b})$. For a natural number $k>2, \mathrm{U}_{1}^{\mathrm{k}}\left(\mathrm{a}_{1}, \cdots, \mathrm{a}_{k}\right)$ is represented by $\nu_{3}\left(a_{1},\left\{a_{2}, \cdots, a_{k}\right\}\right)$. Lemmas 8 and 10 together assure that there exists a system $\mathfrak{I}$, containing a normal $f_{i}^{\prime}$, such that in $\mathfrak{T}, f_{i}^{\prime}(a, b)$ $=\left(\nu_{4}(\mathrm{a}, \mathrm{b})\right)_{\delta(i)}$ is provable. In this system for $i>2, \mathrm{f}_{i}^{\prime}\left(\mathrm{a}_{1},\left\{\mathrm{a}_{2}, \cdots, \mathrm{a}_{k}\right\}\right)$ represents $U_{i}^{k}\left(a_{1}, \cdots, a_{k}\right)$.

If the functor $f$ is defined by a formula

$$
f(\mathfrak{a})=g\left(h_{1}(\mathfrak{a}), \cdots, h_{n}(\mathfrak{a})\right),
$$

where $\mathfrak{a}$ is $\mathrm{a}_{1}, \cdots, \mathrm{a}_{k}$, we must consider subcases according as $k$ and $n$ take values 1,2 , and values greater than 2 . For the case of both greater than 2 , the induction hypothesis assures that there exist normal functors $g^{\prime}$ and $h_{i}^{\prime}$ in some $\mathfrak{T}$, such that in that system we have the provability of

$$
\begin{aligned}
g\left(b_{1}, \cdots, b_{n}\right) & =g^{\prime}\left(b_{1},\left\{b_{2}, \cdots, b_{n}\right\}\right), \\
h_{i}\left(a_{1}, \cdots, a_{k}\right) & =h_{i}^{\prime}\left(a_{1},\left\{a_{2}, \cdots, a_{k}\right\}\right) .
\end{aligned}
$$

Then by Lemma 8 and Lemma 10, there exists a $\mathfrak{T}$ containing a functor $\mathrm{f}^{\prime}$ which represents the term $g^{\prime}\left(h_{1}^{\prime}(c, d),\left\{h_{2}^{\prime}(c, d), \cdots, h_{n}^{\prime}(c, d)\right\}\right)$, and in that system $\mathrm{f}\left(\mathrm{a}_{1}, \cdots, \mathrm{a}_{k}\right)=\mathrm{f}^{\prime}\left(\mathrm{a}_{1},\left\{\mathrm{a}_{2}, \cdots, \mathrm{a}_{k}\right\}\right)$ is provable.

The other cases for the substitution schema which are not already covvered by Lemma 8 follow in a similar manner.

If $f$ is defined by formulas

$$
\begin{aligned}
f(0, \mathfrak{a}) & =g(\mathfrak{a}), \\
f\left(a^{\prime}, \mathfrak{a}\right) & =h(f(a, \mathfrak{a}), \mathfrak{a}, \mathfrak{a}),
\end{aligned}
$$

again we must consider subcases according as $\mathfrak{a}$ abbreviates a sequence of 1,2 , or more than 2 variables. For the case for more than 2 variables, the induction hypothesis gives the provability in some $\mathfrak{T}$ of 


$$
\begin{aligned}
\mathrm{g}\left(\mathrm{a}_{1}, \cdots, \mathrm{a}_{k}\right) & =\mathrm{g}^{\prime}\left(\mathrm{a}_{1},\left\{\mathrm{a}_{2}, \cdots, \mathrm{a}_{k}\right\}\right), \\
\mathrm{h}\left(\mathrm{c}, \mathrm{a}, \mathrm{a}_{1}, \cdots, \mathrm{a}_{k}\right) & =\mathrm{h}^{\prime}\left(\mathrm{c},\left\{\mathrm{a}, \mathrm{a}_{1}, \cdots, \mathrm{a}_{k}\right\}\right) .
\end{aligned}
$$

Then by Lemma 8 and Lemma 10, the term $\nu_{4}\left(d, g^{\prime}\left((b)_{0},\left\{(b)_{1}, \cdots,(b)_{\delta(k)}\right\}\right)\right)$ is represented by a normal $\mathrm{g}^{\prime \prime}$ in some $\mathfrak{I}_{1}$, such that in $\mathfrak{I}_{1}$

$$
g(\mathfrak{a})=g^{\prime \prime}(0,\{\mathfrak{a}\})
$$

is provable.

Then, by the same lemmas, there exists a $\mathfrak{T}_{2}$ containing a normal functor $\mathrm{p}$ such that $\mathrm{p}(\mathrm{a}, \mathrm{b})=\left\{\mathrm{a},(\mathrm{b})_{0}, \cdots,(\mathrm{b})_{\delta(k)}\right\}$ is provable in that system.

If we then add the axioms

$$
\begin{aligned}
f^{\prime}(0, b) & =g^{\prime \prime}(0, b), \\
f^{\prime}\left(a^{\prime}, b\right) & =h^{\prime}\left(f^{\prime}(a, b), p(a, b)\right)
\end{aligned}
$$

to the set-theoretic sum of the axioms of $\mathfrak{T}_{1}$ and $\mathfrak{T}_{2}$, we may show by the equality schema 23 and Schema 13 that the formula $f(a, a)=f^{\prime}(a,\{\mathfrak{a}\})$ is provable in the system $\mathfrak{T}$ so formed.

The cases for 1 and 2 variables in the sequence $\mathfrak{a}$ are similar, as is the case of the recursion schema in which the variables $\mathfrak{a}$ do not appear.

\section{PART III}

In Part III we continue with the problem of formalizing the theory of recursive functions.

Instead of arithmetizing the formalization of recursion equations by Gödel's method of numbering, as Kleene did in the construction of his predicates $T_{k}\left(e, a_{1}, \cdots, a_{k}, b\right)[11 ; 13]$, we use a method based on the normal form for equations defining primitive recursive functions of two variables which was given in Part II. These functions can be enumerated in a straightforward manner. The enumeration can then be extended, in effect, to the (non-primitive) general and partial recursive functions with the aid of the existential quantifier.

This treatment does not supplant the use of Kleene's predicates $T_{k}\left(e, a_{1}, \cdots, a_{k}, b\right)$ for the purpose of showing the possibility of representation of arbitrary general and partial recursive functions $[11 ; 13]$. It does afford, for each $k$, a predicate $R_{k}\left(e, a_{1}, \cdots, a_{k}, b\right)$ having properties similar to $T_{k}\left(e, a_{1}, \cdots, a_{k}, b\right)$, and in terms of which it is much easier to formalize the theory for any particular general and partial recursive functions.

The following sections contain a formal development of the theory of the predicates $R_{k}\left(e, a_{1}, \cdots, a_{k}, b\right)$. To this end, we first introduce a formal system of intuitionistic number theory $S_{3}$ containing formulas $\mathrm{R}_{k}\left(\mathrm{e}, \mathrm{a}_{1}, \cdots, \mathrm{a}_{k}, \mathrm{~b}\right)$. Next we demonstrate an equivalence of formal systems of intuitionistic number theory containing $S_{3}$ to $S_{3}$. Finally we develop an abbreviation for the representation of functions in $S_{3}$. 
8. The representing predicate. We shall construct a system $S_{3}$ containing a functor $\psi$, such that for every normal recursive functor $\mathrm{f}$ of a system $\mathfrak{S}$ containing $S_{3}$ there exists a numeral $n$, such that the formula $\exists y \psi(n, a, b, c, y)$ $=0 \equiv \mathrm{f}(\mathrm{a}, \mathrm{b})=\mathrm{c}$ is provable in $\mathfrak{S}$.

Let us consider the definition of normal recursive functor. We might describe every normal functor as one of the fundamental symbols' $\nu_{i}$, where $i=1, \cdots, 4$, or as the value of one of two syntactical functions $S b\left(f_{1}, f_{2}, f_{3}\right)$ or $\operatorname{Rec}\left(\mathrm{f}_{1}, \mathrm{f}_{2}, \mathrm{f}_{3}\right)$ which takes functors as arguments and has functors as values. The value of $S b\left(f_{1}, f_{2}, f_{3}\right)$ is the functor which has as its defining equation an axiom of the form specified by the substitution schema, (a) (1), where $f_{1}, f_{2}$, and $\mathrm{f}_{3}$ are previously defined functors. The value of $\operatorname{Rec}\left(\mathrm{f}_{1}, \mathrm{f}_{2}, \mathrm{f}_{3}\right)$ is the functor defined by equations of the form of the recursion schema (a) (2).

If we utilize a method of Gödel numbering to assign certain numbers to the functors $\nu_{i}(i=1, \cdots, 4)$ and choose suitable number-theoretic listing functions to represent $S b$ and $R e c$, we can represent every normal recursive functor by a natural number. Thus we shall establish a one-to-one correspondence between certain natural numbers and recursive functions. This approach obviates the necessity for arithmetizing the syntactical concepts of variable, numeral, and formula to arithmetize the notion of deduction of a value for a recursive function.

We continue the intuitive discussion using our formal notation for the corresponding number-theoretic functions. Let correspond to the functors $\nu_{i}$, where $i=1, \cdots, 4$, the natural numbers $1, \cdots, 4$. If the natural numbers $n_{1}, n_{2}, n_{3}$ correspond to the functors $f_{1}, f_{2}, f_{3}$, then let correspond to the value of $S b\left(\mathrm{f}_{1}, \mathrm{f}_{2}, \mathrm{f}_{3}\right)$ the natural number $\left\{5, n_{1}, n_{2}, n_{3}\right\}$, and to the value of $\operatorname{Rec}\left(\mathrm{f}_{1}, \mathrm{f}_{2}, \mathrm{f}_{3}\right)$ let correspond $\left\{6, n_{1}, n_{2}, n_{3}\right\}$.

If the number $n$ represents the functor $\mathrm{f}$ in the way which we have indicated above, then to a formula

$$
\mathrm{f}(\boldsymbol{m}, \mathbf{l})=\boldsymbol{k}
$$

let correspond a number $\{n, m, l, k, h\}$ where $h$ is a deduction number which we consider next $\left({ }^{10}\right)$.

The deduction number, $h$, for a formula is defined inductively with respect to the number $n$ of the functor, with a subsidiary inductive clause in case the number of the functor corresponds to a value of $R e c$.

If $n$ represents one of the functors $\nu_{i}$, then $h$ is chosen as 0 .

If $n$ represents a value of $S b\left(\mathrm{f}_{1}, \mathrm{f}_{2}, \mathrm{f}_{3}\right)$, then for an equation $\mathrm{f}(m, 1)=k$, there is an equivalent set of equations

$$
\mathrm{f}_{1}(\mathrm{~m}, 1)=k_{1}, \quad \mathrm{f}_{2}(\mathrm{~m}, 1)=k_{2}, \quad \mathrm{f}_{3}\left(k_{1}, k_{2}\right)=k .
$$

Suppose these have the respective deduction numbers $h_{1}, h_{2}, h_{3}$, then $h$ is

(10) So called by analogy with the Gödel number of a formal deduction in Kleene's treatment of the predicate $S_{n}[13, \S 4]$. 
chosen as the number

$$
\left\{\left\{n_{1}, m, l, k_{1}, h_{1}\right\},\left\{n_{2}, m, l, k_{2}, h_{2}\right\},\left\{n_{3}, k_{1}, k_{2}, k, h_{3}\right\}\right\} .
$$

If $n$ represents a value of $\operatorname{Rec}\left(\mathrm{f}_{1}, \mathrm{f}_{2}, \mathrm{f}_{3}\right)$ for an equation $\mathrm{f}(m, l)=k$, we consider two cases as $m=0$ or as $m \neq 0$. If $m=0$ and $h_{1}$ is the deduction number of $\mathrm{f}_{1}(0, l)=k$, then $h$ is defined as $\left\{n_{1}, 0, l, k, h_{1}\right\}$. If $m \neq 0$, and the deduction numbers of the formulas

$$
\mathrm{f}(\delta(\mathrm{m}), I)=k_{1}, \quad \mathrm{f}_{2}(\delta(m), I)=k_{2}, \quad \mathrm{f}_{3}\left(k_{1}, k_{2}\right)=k
$$

are $h_{1}, h_{2}, h_{3}$, then $h$ is chosen as

$$
\left\{\left\{n, \delta(m), l, k_{1}, h_{1}\right\},\left\{n_{2}, \delta(m), l, k_{2}, h_{2}\right\},\left\{n_{3}, k_{1}, k_{2}, k, h_{3}\right\}\right\} .
$$

The formula $\psi(p)=0$, where $\psi$ is the functor to be defined in Lemma 15, will be the formal counterpart of the following intuitive statement when $(p)_{0}$ is the number of a function: The function numbered $(p)_{0}$ for the arguments $(p)_{1}$ and $(p)_{2}$ has the value $(p)_{3}$ and the deduction number $(p)_{4}$. (In the case in which $(p)_{00}=6$ and $(p)_{1}=0$, the interpretation does not require that. $(p)_{0}$ be the number of a primitive recursive function, only that $(p)_{01}$ be such a number.)

We now turn to the problem of representing general and partial recursive functions. Let $A(c, a, d, b)$ be a formula of Class $\Gamma$ containing exactly the free variables $\mathrm{c}, \mathfrak{a}, \mathrm{d}, \mathrm{b}$, where $\mathfrak{a}$ is $\mathrm{a}_{1}, \cdots, \mathrm{a}_{k}(k \geqq 1)$. If the formula

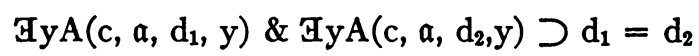

is provable in some system $\mathfrak{S}$, then the formula $\operatorname{\exists yA}(c, \mathfrak{a}, d, y)$ specifies a function $\lambda c a_{1} \cdots a_{k} f\left(c, a_{1}, \cdots, a_{k}\right)$, which is defined for natural numbers $m, n_{1}, \cdots, n_{k}$, and has the value $n$, if the formula $\operatorname{\exists yA}\left(m, n_{1}, \cdots, n_{k}, n, y\right)$ is provable in $\mathfrak{S}$. Such functions will include general and partial recursive functions $[11 ; 13]$.

Let $\mathrm{g}$ be the functor corresponding to $\mathrm{A}(\mathrm{c}, \mathfrak{a}, \mathrm{d}, \mathrm{b})$ under the correspondence mentioned in Lemma 11; then, by Lemmas 13 and 14, under the interpretation, there exists a natural number $p$ such that the relation $g\left(m, n_{1}, \cdots, n_{k}, n, l\right)=0$ will hold if and only if there exists a number $q$ such that $\psi\left(\left\{p, m,\left\{n_{1}, \cdots, n_{k}, n, l\right\}, 0, q\right\}\right)=0$. We shall introduce $\mathrm{p}_{k}\left(\{\boldsymbol{p}, \boldsymbol{m}\}, \boldsymbol{n}_{\mathbf{1}}, \cdots, \boldsymbol{n}_{k},\{\boldsymbol{n}, \mathbf{1}, \boldsymbol{q}\}\right)$ as an abbreviation for a formula equivalent to $\psi\left(\left\{p, m,\left\{n_{1}, \cdots, n_{k}, n, 1\right\}, 0, q\right\}\right)=0$.

Then the number $\{p, m\}$ will be taken as the number of the function $\lambda a_{1} \cdots a_{k} f\left(m, a_{1}, \cdots, a_{k}\right)$, and $\{n, l, q\}$ as a deduction number of this function for the arguments $n_{1}, \cdots, n_{k}$ in case $g\left(m, n_{1}, \cdots, n_{k}, n, l\right)=0$.

Using this method of numbering functions, we shall have a convenient method of characterizing the class of functions formed by $\lambda a_{1} \cdots a_{k} f\left(m, a_{1}, \cdots, a_{k}\right)$ as the parameter $m$ varies. This characterization will afford an easy means of formalizing functions serving the same purpose as the functions $S_{f}^{\natural}$, which 
were of importance in the theory of Part I.

We shall introduce $\mathrm{R}_{k}(\mathrm{e}, \mathfrak{a}, \mathrm{d})$ as an abbreviation for a formula equivalent to $\mathrm{P}_{k}(\mathrm{e}, \mathfrak{a}, \mathrm{d}) \& \forall \mathrm{x}\left(\mathrm{P}_{k}(\mathrm{e}, \mathfrak{a}, \mathrm{x}) \supset \mathrm{d} \leqq \mathrm{x}\right)$, so that $\mathrm{R}_{k}(\mathrm{e}, \mathfrak{a}, \mathrm{d}) \supset \mathrm{P}_{k}(\mathrm{e}, \mathfrak{a}, \mathrm{d})$ and $\exists \mathrm{BP}_{k}(\mathrm{e}, \mathfrak{a}, \mathrm{x}) \supset \mathrm{GxR}_{k}(\mathrm{e}, \mathfrak{a}, \mathrm{x})$ will be provable; but for each choice of $\boldsymbol{r}, \boldsymbol{n}_{1}, \cdots, \boldsymbol{n}_{k}$, if $\mathrm{R}_{k}\left(\boldsymbol{r}, \boldsymbol{n}_{1}, \cdots, \boldsymbol{n}_{k}, \boldsymbol{s}\right)$ is provable, then it will be provable for a unique $s$. The formula $\mathrm{R}_{k}\left(r, n_{1}, \cdots, n_{k}, s\right) \&(s)_{0}=n$ will be the formal counterpart of the intuitive statement "The function numbered $r$ (under our second method of numbering) for the arguments $n_{1}, \cdots, n_{k}$ has a least deduction number $s$ and the value $n . "$

Because of its frequent occurrence in the following work, we shall abbreviate a term of the form (a) $)_{0}$ with the expression [a].

We shall abbreviate a term of the form $\left(\cdots(s)_{t_{1}} \cdots\right)_{t_{k}}$ with the expression $(s)_{t_{1}} \ldots_{t_{k}}$. The decimal abbreviation for numerals will be used in the subscript position only for numerals $n$ such that $n \leqq 9$. Thus (s) $)_{213}$ abbreviates $\left(\left((s)_{2}\right)_{1}\right)_{3}$.

LEMMA 15. There exists a system of recursive number theory $S_{3}$, such that the formulas 1-10 are provable in $S_{3}$, where in 8 and 9 , for each integer $k>0$, $\mathrm{P}_{k}(\mathrm{c}, \mathfrak{a}, \mathrm{d})$ and $\mathrm{R}_{k}(\mathrm{c}, \mathfrak{a}, \mathrm{d})$ are abbreviations for $\mathrm{p}_{k}(\mathrm{c}, \mathfrak{a}, \mathrm{d})=0$ and $\mathrm{r}_{k}(\mathrm{c}, \mathfrak{a}, \mathrm{d})=0$, respectively, where $\mathrm{p}_{k}$ and $\mathrm{r}_{k}$ are functors of $S_{3}$.

The existence of defining axioms for the functors appearing in 1-6.2 follows immediately from Lemma 11.

1. $\psi_{1}(a)=0 \equiv a=\left\{1,(a)_{1},(a)_{2}, 0,0\right\}$.

2. $\psi_{2}(a)=0 \equiv a=\left\{2,(a)_{1},(a)_{2},\left((a)_{1}\right)^{\prime}, 0\right\}$.

3. $\psi_{3}(a)=0 \equiv a=\left\{3,(a)_{1},(a)_{2},(a)_{1}, 0\right\}$.

4. $\psi_{4}(a)=0 \equiv a=\left\{4,(a)_{1},(a)_{2},(a)_{2}, 0\right\}$.

5. $\sigma(a)=0 \equiv a=\left\{\left\{5,(a)_{01},(a)_{02},(a)_{03}\right\},(a)_{1},(a)_{2},(a)_{3},\{s, t, u\}\right\}$, where the following abbreviations are made:

$$
\begin{aligned}
\mathrm{s} & =\left\{(\mathrm{a})_{01},(\mathrm{a})_{1},(\mathrm{a})_{2},(\mathrm{a})_{403},(\mathrm{a})_{404}\right\} \\
\mathrm{t} & =\left\{(\mathrm{a})_{02},(\mathrm{a})_{1},(\mathrm{a})_{2},(\mathrm{a})_{413},(\mathrm{a})_{414}\right\} \\
\mathrm{u} & =\left\{(\mathrm{a})_{03},(\mathrm{a})_{403},(\mathrm{a})_{413},(\mathrm{a})_{3},(\mathrm{a})_{424}\right\} .
\end{aligned}
$$
(a) 44$\}$.

6.1. $\rho_{1}(a)=0 \equiv a=\left\{\left\{6,(a)_{01},(a)_{02},(a)_{03}\right\}, 0,(a)_{2},(a)_{3},\left\{(a)_{01}, 0,(a)_{2},(a)_{3}\right.\right.$,

6.2. $\rho_{2}(a)=0 \equiv a=\left\{\left\{6,(a)_{01},(a)_{02},(a)_{03}\right\},(a)_{1},(a)_{2},(a)_{3},\{s, t, u\}\right\}$, where the following abbreviations are made:

$$
\begin{aligned}
\mathrm{s} & =\left\{\mathrm{a}_{0}, \delta\left((\mathrm{a})_{1}\right),(\mathrm{a})_{2},(\mathrm{a})_{403},(\mathrm{a})_{404}\right\} \\
\mathrm{t} & =\left\{(\mathrm{a})_{02}, \delta\left((\mathrm{a})_{1}\right),(\mathrm{a})_{2},(\mathrm{a})_{413},(\mathrm{a})_{414}\right\} \\
\dot{\mathrm{u}} & =\left\{(\mathrm{a})_{03},(\mathrm{a})_{403},(\mathrm{a})_{413},(\mathrm{a})_{3},(\mathrm{a})_{423}\right\} .
\end{aligned}
$$

7.1. $\phi_{0}(a)=\psi_{1}(a) \cdot \psi_{2}(a) \cdot \psi_{3}(a) \cdot \psi_{4}(a)$. 
Introducing auxiliary functors $\phi_{1}, \phi_{2}$, and $\phi_{3}$ for typographical convenience, we have the following course-of-values recursion axioms for $\psi$. Observe that the formulas $\left(\mathrm{a}^{\prime}\right)_{4}<\mathrm{a}^{\prime}$ and $\left(\mathrm{a}^{\prime}\right)_{4 i}<\mathrm{a}^{\prime}$, where $i=0,1,2$, are provable in $S_{2}$. This follows from Lemma 10,10.6, 10.1, 8.5 with the aid of 9.9, 1.0, and Axiom 15.

7.2. $\phi_{1}(a)=\sigma(a)+\psi\left((a)_{40}\right)+\psi\left((a)_{41}\right)+\psi\left((a)_{42}\right)$.

7.3. $\phi_{2}(\mathrm{a})=\rho_{1}(\mathrm{a})+\psi\left((\mathrm{a})_{4}\right)$.

7.4. $\phi_{3}(\mathrm{a})=\rho_{2}(\mathrm{a})+\beta\left(\epsilon\left((\mathrm{a})_{1}, 0\right)\right)+\psi\left((\mathrm{a})_{40}\right)+\psi\left((\mathrm{a})_{41}\right)+\psi\left((\mathrm{a})_{42}\right)$.

7.5. $\psi(0)=1$.

7.6. $\psi\left(\mathrm{a}^{\prime}\right)=\phi_{0}\left(\mathrm{a}^{\prime}\right) \cdot \phi_{1}\left(\mathrm{a}^{\prime}\right) \cdot \phi_{2}\left(\mathrm{a}^{\prime}\right) \cdot \phi_{3}\left(\mathrm{a}^{\prime}\right)$.

In Formula 8, below, the right member is a formula of Class $\Gamma$; hence, by Lemma 11 , there exists a functor $\mathrm{p}_{k}$ of some formal system $\mathfrak{S}_{1}$, such that, if $\mathrm{P}_{k}(\mathrm{c}, \mathfrak{a}, \mathrm{d})$ abbreviates $\mathrm{p}_{k}(\mathrm{c}, \mathfrak{a}, \mathrm{d})=0$, then Formula 8 will be provable in $\mathfrak{S}_{1}$. It will be understood that $\mathrm{P}_{k}(\mathrm{c}, \mathfrak{a}, \mathrm{d})$ is such an abbreviation, and that $S_{\mathbf{z}}$ is chosen to contain $\widetilde{S}_{1}$.

8. $P_{k}(c, a, d) \equiv \psi\left(\left\{(c)_{0},(c)_{1},\left\{a,(d)_{0},(d)_{1}\right\}, 0,(d)_{2}\right\}\right)=0 \& c=\left\{(c)_{0},(c)_{1}\right\}$ $\& d=\left\{(d)_{0},(d)_{1},(d)_{2}\right\}$.

Since $\mathrm{P}_{k}(\mathrm{c}, \mathfrak{a}, \mathrm{d})$ is a formula of Class $\Gamma$, the formula $\mathrm{P}_{k}(\mathrm{c}, \mathfrak{a}, \mathrm{d}) \& \forall \mathrm{x}(\mathrm{x}<\mathrm{d}$ $\left.\supset>\mathrm{P}_{k}(\mathrm{c}, \mathfrak{a}, \mathrm{x})\right)$ is also of Class $\Gamma$. By Lemma 10, 8.6, 9.1, 9.7, 1.0 and the predicate calculus, we have the provability in some system containing $\Im_{1}$ of

$$
\mathrm{Vx}\left(\mathrm{P}_{k}(\mathrm{c}, \mathfrak{a}, \mathrm{x}) \supset \mathrm{d} \leqq \mathrm{x}\right) \equiv \mathrm{Vx}\left(\mathrm{x}<\mathrm{d} \supset>\mathrm{P}_{k}(\mathrm{c}, \mathfrak{a}, \mathrm{x})\right) .
$$

Again by Lemma 11, there exists a functor $r_{k}$ of some formal system $\Im_{2}$, such that, if $\mathrm{R}_{k}(\mathrm{c}, \mathfrak{a}, \mathrm{d})$ abbreviates $\mathrm{r}_{k}(\mathrm{c}, \mathfrak{a}, \mathrm{d})=0$, Formula 9 will be provable in $\widetilde{S}_{2}$. It will be understood that $\mathrm{R}_{k}(\mathrm{c}, \mathfrak{a}, \mathrm{d})$ is such an abbreviation and that $S_{3}$ is chosen to contain $\mathfrak{S}_{2}$.

9. $\mathrm{R}_{k}(\mathrm{c}, \mathfrak{a}, \mathrm{d}) \equiv \mathrm{P}_{k}(\mathrm{c}, \mathfrak{a}, \mathrm{d}) \& \forall \mathrm{x}\left(\mathrm{P}_{k}(\mathrm{c}, \mathfrak{a}, \mathrm{x}) \supset \mathrm{d} \leqq \mathrm{x}\right)$.

By the abbreviation previously stated (and $S_{1}$ Axiom 16):

10. $[\mathrm{d}]=(\mathrm{d})_{0}$.

CoROLlaRy. The following formulas are provable in $S_{3}$ :

1. $\psi_{i}(\{i, \mathrm{a}, \mathrm{b}, \mathrm{c}, 0\})=0 \equiv \mathrm{Gx} \psi(\{i, \mathrm{a}, \mathrm{b}, \mathrm{c}, \mathrm{x}\})=0$, where $i=1, \cdots, 4$.

2. $\exists x \phi_{1}\left(\left\{\left\{5, n_{1}, n_{2}, n_{3}\right\}, a, b, c, x\right\}\right)=0 \equiv \exists x \psi\left(\left\{\left\{5, n_{1}, n_{2}, n_{3}\right\}, a, b, c, x\right\}\right)$ $=0$.

3. $\exists x \psi\left(\left\{\mathrm{n}_{1}, 0, \mathrm{~b}, \mathrm{c}, \mathrm{x}\right\}\right)=0 \equiv \exists \times \psi\left(\left\{\left\{6, \mathrm{n}_{1}, \mathrm{n}_{2}, \mathrm{n}_{3}\right\}, 0, \mathrm{~b}, \mathrm{c}, \mathrm{x}\right\}\right)=0$.

4. $\exists x \phi_{3}\left(\left\{\left\{6, n_{1}, n_{2}, n_{3}\right\}, a^{\prime}, b, c, x\right\}\right)=0 \equiv \exists x \psi\left(\left\{\left\{6, n_{1}, n_{2}, n_{3}\right\}, a^{\prime}, b, c, x\right\}\right)$ $=0$.

5. $\mathrm{R}_{k}\left(\mathrm{c}, \mathfrak{a}, \mathrm{d}_{1}\right) \& \mathrm{R}_{k}\left(\mathrm{c}, \mathfrak{a}, \mathrm{d}_{2}\right) \supset \mathrm{d}_{1}=\mathrm{d}_{2}$.

We consider in detail only part of the proof of Formula 3, namely, the proof of the formula

$$
\operatorname{Hx} \psi\left(\left\{\left\{6, \mathrm{n}_{1}, \mathrm{n}_{2}, \mathrm{n}_{3}\right\}, 0, \mathrm{~b}, \mathrm{c}, \mathrm{x}\right\}\right)=0 \supset \exists \times \psi\left(\left\{\mathrm{n}_{1}, 0, \mathrm{~b}, \mathrm{c}, \mathrm{x}\right\}\right)=0 .
$$

The remainder of the proof of 3 and the proofs of the other formulas are con- 
structed similarly (and for the most part more simply).

Let $t$ abbreviate $\left\{\left\{6, n_{1}, n_{2}, n_{3}\right\}, 0, b, c, d\right\}$; then the following formulas are provable in $S_{3}$.

(1) $7 \mathrm{t}=0$. Lemma 10,10.1, 10.7; $S_{1}$ Axiom 15.

(2) $7 \mathrm{a}=0 \supset \mathrm{a}=(\delta(\mathrm{a}))^{\prime}$. Schema 13, Axiom 16; Lemma 10, 4.2.

(3) $\psi(\mathrm{t})=\phi_{0}(\mathrm{t}) \cdot \phi_{1}(\mathrm{t}) \cdot \phi_{2}(\mathrm{t}) \cdot \phi_{3}(\mathrm{t})$. (1), (2); Schema 23; Lemma 15, 7.6.

(4) $\psi(\mathrm{t})=0 \equiv \phi_{0}(\mathrm{t})=0 \bigvee \phi_{1}(\mathrm{t})=0 \bigvee \phi_{2}(\mathrm{t})=0 \bigvee \phi_{3}(\mathrm{t})=0$. (3); Lemma 10, 1.7.

(5) $7(t)_{0}=0$. Lemma 10, 10.1, 10.7; $\mathrm{S}_{1}$ Axiom 15.

(6) $6<(\mathrm{t})_{0} .(5)$; Lemma $10,10.6,10.7$

In Formulas (7)-(10), $i=1, \cdots, 4$.

(7) $i<6$. Lemma $10,8.3,8.5$.

(8) $7(t)_{0}=i$. (6), (7); Lemma 10, 8.5, 8.8.

(9) $\psi_{i}(t)=0 \supset(t)_{0}=i$. Lemma 15, 1-4; Lemma 10, 10.7.

(10) $7 \psi_{i}(\mathrm{t})=0$. (8), (9).

(11) $7 \phi_{0}(t)=0$. (10); Lemma 15, 7.1; Lemma 10, 1.7.

(12) $7(t)_{00}=5$. Lemma $10,10.7,8.3,8.5,8.8$.

(13) $\sigma(t)=0 \supset(t)_{00}=5$. Lemma 15,5 ; Lemma 10, 10.7.

(14) $7 \phi_{1}(t)=0$. (12), (13); Lemma 15, 7.2; Lemma 10, 1.8 .

(15) $\epsilon\left((t)_{1}, 0\right)=0$. Lemma 10, 10.7, 6.2.

(16) $7 \beta\left(\epsilon\left((t)_{1}, 0\right)\right)=0$. (15); Lemma 10, 3.3.

(17) $7 \phi_{3}(t)=0$. (16); Lemma 15, 7.4; Lemma 10, 1.8 .

(18) $\psi(\mathrm{t})=0 \supset \phi_{2}(\mathrm{t})=0$. (4), (11), (14), (17).

(19) $\psi(\mathrm{t})=0 \supset \psi(\mathrm{d})=0$. (18); Lemma 15, 7.3; Lemma 10, 10.7.

(20) $\psi(t)=0 \supset d=\left\{n_{1}, 0, b, c,(d)_{4}\right\} .(18) ;$ Lemma 15, 7.3, 6.1; Lemma 10, 10.7 .

(21) $\psi(\mathrm{t})=0 \supset \psi\left(\left\{\mathrm{n}_{1}, 0, \mathrm{~b}, \mathrm{c},(\mathrm{d})_{4}\right\}\right)=0 .(19),(20)$.

The desired formula is obtained from (21) by introduction of existential quantifiers.

LEMMA 16. For every normal recursive functor $\mathrm{f}$ of a system $\subseteq$ there exists a numeral $n$ such that the formula

$$
f(a, b)=c \equiv \mathcal{H} \psi(\{n, a, b, c, x\})=0
$$

is provable in S.

The lemma is proved by induction on the number of functors upon which $f$ depends.

For the basis of the induction, suppose $f$ depends on no other functors; then there are four cases, one for each of the functors $\nu_{i}$, as $i=1, \cdots, 4$. The proofs for the separate cases are similar, so we outline only the one of them for $\nu_{4}$.

The following formulas are provable in $\mathfrak{S}$ provided $\nu_{4}$ is a functor of $\mathfrak{S}$. After each formula there is an indication of the more important formulas used in its deduction. 
1. $\nu_{4}(\mathrm{a}, \mathrm{b})=\mathrm{c} \equiv \mathrm{b}=\mathrm{c}$. Defining axiom for $\nu_{4}$, Schema 23 .

2. $\mathrm{b}=\mathrm{c} \equiv \psi_{4}(\{4, \mathrm{a}, \mathrm{b}, \mathrm{c}, 0\})=0$. Lemma 15,4 ; Lemma 10, 10.7; Schema 23 .

3. $\psi_{4}(\{4, a, b, c, 0\})=0 \equiv \exists \times \psi(\{4, a, b, c, x\})=0$. Corollary, Lemma 15, 1 .

4. $\nu_{4}(\mathrm{a}, \mathrm{b})=\mathrm{c} \equiv \exists \mathrm{Jx} \psi(\{4, \mathrm{a}, \mathrm{b}, \mathrm{c}, \mathrm{x}\})=0.1,2,3$.

The induction step is divided into two cases. For the first case, assume that $f$ has as its defining axiom in $\subseteq$ a formula of the form (a) (1).

Then the induction hypothesis assures the existence of numerals $\boldsymbol{n}_{\mathbf{1}}, \boldsymbol{n}_{\mathbf{2}}, \boldsymbol{n}_{\mathbf{3}}$ such that the following formulas are provable in $\mathfrak{S}$ :

$$
\begin{aligned}
\mathrm{f}_{1}(\mathrm{a}, \mathrm{b}) & =\mathrm{c}_{1} \equiv \mathrm{H}_{\mathrm{x}} \psi\left(\left\{\mathrm{n}_{1}, \mathrm{a}, \mathrm{b}, \mathrm{c}_{1}, \mathrm{x}_{1}\right\}\right)=0 . \\
\mathrm{f}_{2}(\mathrm{a}, \mathrm{b}) & =\mathrm{c}_{2} \equiv \mathrm{Gx}_{2} \psi\left(\left\{n_{2}, \mathrm{a}, \mathrm{b}, \mathrm{c}_{2}, \mathrm{x}_{2}\right\}\right)=0 . \\
\mathrm{f}_{3}\left(\mathrm{c}_{1}, \mathrm{c}_{2}\right) & =\mathrm{c} \equiv \mathrm{Gx}_{3} \psi\left(\left\{n_{3}, c_{1}, c_{2}, c, \mathrm{x}_{3}\right\}\right)=0 .
\end{aligned}
$$

Let $n$ abbreviate $\left\{5, n_{1}, n_{2}, n_{3}\right\}$; then the following formulas are provable in $\subseteq$ :

1. $f(a, b)=c \equiv \exists y_{1} \exists y_{2}\left(f_{1}(a, b)=y_{1} \& f_{2}(a, b)=y_{2} \& f\left(y_{1}, y_{2}\right)=c\right)$. Defining axiom for $f$, Schema 23 .

2. $f(a, b)=c \equiv \exists y_{1} \exists y_{2} \exists x_{1} \exists x_{2} \exists x_{3}\left(\psi\left(\left\{n_{1}, a, b, y_{1}, x_{1}\right\}\right)=0 \& \psi\left(\left\{n_{2}, a, b, y_{2}, x_{2}\right\}\right)\right.$ $\left.=0 \& \psi\left(\left\{n_{3}, \mathrm{y}_{1}, \mathrm{y}_{2}, \mathrm{c}, \mathrm{x}_{3}\right\}\right)=0\right)$. (1), (i), (ii), (iii).

3. $\sigma\left(\left\{n, a, b, c,\left\{\left\{n_{1}, a, b, c_{1}, d_{1}\right\},\left\{n_{2}, a, b, c_{2}, d_{2}\right\},\left\{n_{3}, c_{1}, c_{2}, c, d_{3}\right\}\right\}\right\}\right)$ $=0$. Lemma 15, 5; Lemma 10, 10.7; Schema 23 .

4. $f(a, b)=c \equiv \exists y_{1} \exists y_{2} \exists x_{1} B x_{2} 3 x_{3} \phi_{1}(t)=0$, where $t$ is the result of replacing the free variables $c_{1}, c_{2}, d_{1}, d_{2}, d_{3}$ by $y_{1}, y_{2}, x_{1}, x_{2}, x_{3}$, respectively, in the expression enclosed in parentheses in 3. 2 ; 3 ; Lemma 15, 7.2; Lemma 10, 1.8, 10.7 ; Schema 23.

5. $\mathrm{f}(\mathrm{a}, \mathrm{b})=\mathrm{c} \equiv \mathrm{G} \times \phi_{1}(\{\mathrm{n}, \mathrm{a}, \mathrm{b}, \mathrm{c}, \mathrm{x}\})=0.4 ;$ Lemma $15,5,7.2$

6. $\mathrm{f}(\mathrm{a}, \mathrm{b})=\mathrm{c} \equiv \overline{\mathrm{Jx}} \psi(\{\mathrm{n}, \mathrm{a}, \mathrm{b}, \mathrm{c}, \mathrm{x}\})=0.5$; Corollary to Lemma 15, 2.

For the second case of the induction step, suppose $f$ is defined by axioms of the form (a) (2). For this case we show that a formula $\forall y(f(a, b)=y$ $\equiv \exists \times \psi(\{n, a, b, y, x\})=0)$ abbreviated $\operatorname{VyA}(a, y)$ is provable in $\subseteq$. The proof is by an application of the induction Schema 13 of $S_{1}$, induction on a.

The induction hypothesis of the lemma assures the provability in $\subseteq$ of the following formulas.

$$
\begin{aligned}
\mathrm{f}_{1}(0, b) & =\mathrm{c} \equiv \operatorname{Hx} \psi\left(\left\{n_{1}, 0, b, c, x\right\}\right)=0 . \\
\mathrm{f}_{2}(\mathrm{a}, \mathrm{b}) & =\mathrm{c}_{2} \equiv \operatorname{Hx}_{2} \psi\left(\left\{n_{2}, \mathrm{a}, \mathrm{b}, \mathrm{c}_{2}, \mathrm{x}_{2}\right\}\right)=0 . \\
\mathrm{f}_{3}\left(\mathrm{c}_{1}, \mathrm{c}_{2}\right) & =\mathrm{c} \equiv \operatorname{Gx}_{3} \psi\left(\left\{n_{3}, c_{1}, c_{2}, \mathrm{c}, \mathrm{x}_{3}\right\}\right)=0 .
\end{aligned}
$$
in $\mathfrak{S}$.

If $n$ abbreviates $\left\{6, n_{1}, n_{2}, n_{3}\right\}$, then the following formulas are provable

1. $f(0, b)=c \equiv f_{1}(0, b)=c$. Defining axioms for $f$, Schema 23 .

2. $\mathrm{f}(0, \mathrm{~b})=\mathrm{c} \equiv \mathrm{Gx} \psi\left(\left\{n_{1}, 0, \mathrm{~b}, \mathrm{c}, \mathrm{x}\right\}\right)=0.1$, (i).

3. $\exists x \psi\left(\left\{n_{1}, 0, b, c, x\right\}\right)=0 \equiv \exists \times \psi(\{n, 0, b, c, x\})=0$. Corollary to Lemma 15,3 . 
4. $\forall y A(0, y) .2,3$.

5. $f\left(a^{\prime}, b\right)=c \equiv \exists y_{1} \exists y_{2}\left(f(a, b)=y_{1} \& f_{2}(a, b)=y_{2} \& f_{3}\left(y_{1}, y_{2}\right)=c\right)$. Defining axioms for $f$, Schema 23 .

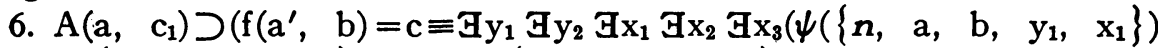
$\left.\left.=0 \& \psi\left(\left\{n_{2}, a, b, y_{2}, x_{2}\right\}\right)=0 \& \psi\left(\left\{n_{3}, y_{1}, y_{2}, c, x_{3}\right\}\right)=0\right)\right) .4,5$, (ii), (iii).

7. $\rho_{2}\left(\left\{n, a^{\prime}, b, c,\left\{\left\{n, a, b, c_{1}, d_{1}\right\},\left\{n_{2}, a, b, c_{2}, d_{2}\right\},\left\{n_{3}, c_{1}, c_{2}, c, d_{3}\right\}\right\}\right\}\right)$ $=0$. Lemma 15, 6.2; Lemma 10, 4.2.

8. $\beta\left(\epsilon\left(a^{\prime}, 0\right)\right)=0$. Lemma $10,6.2$. 3.3.

9. $A\left(a, c_{1}\right) \supset\left(f\left(a^{\prime}, b\right)=c \equiv \exists x \Phi_{3}\left(\left\{n, a^{\prime}, b, c, x\right\}\right)=0\right) .6,7,8$; Lemma 15, 7.4, 6.2; Schema 23.

10. $A\left(a, c_{1}\right) \supset\left(f\left(a^{\prime}, b\right)=c \equiv \exists x \psi\left(\left\{n, a^{\prime}, b, c, x\right\}\right)=0\right)$. 9; Corollary to Lemma 15, 4.

11. $\forall y A(a, y) \supset \forall y A\left(a^{\prime}, y\right) .10$.

12. $\forall y A(a, y) .4,11$; Schema 13 .

13. $f(a, b)=c \equiv \exists x \psi(\{n, a, b, c, x\})=0.12$.

For the statement of Lemmas 17 and 18 and their corollaries, we introduce two abbreviations.

We agree in these lemmas and their applications to allow small German letters to abbreviate empty sequences of variables as well as sequences of variables, with the exception of the letter $\mathfrak{a}$ as used in formulas $\mathrm{P}_{k}(\mathrm{e}, \mathfrak{a}, \mathrm{d})$, $\mathrm{R}_{k}(\mathrm{e}, \mathfrak{a}, \mathrm{d}$ ), and so on. (To allow $\mathfrak{a}$ to represent the empty sequence, with $k=0$, would amount to considering functions of 0 arguments. This would be entirely possible, but for our present purposes there is no advantage, and it would require the consideration of an additional case in the following lemmas.)

Then, if $Q$ is either $\exists$ or $\forall$ and $y$ is $y_{1} \cdots y_{l}, Q y$ abbreviates $Q y_{1} \cdots Q y_{l}$. In case $\mathfrak{y}$ is empty, $Q \mathfrak{y}$ abbreviates an empty sequence of symbols.

We define the abbreviation $\eta(m, e)$ as follows: If $e$ is the empty sequence, $\eta(\mathrm{m}, \mathrm{e})$ is $\mathrm{m}$. If $\mathrm{e}$ is $\mathrm{e}_{1}, \eta(\mathrm{m}, \mathrm{e})$ is $\left\{\mathrm{m}, \mathrm{e}_{1}\right\}$. If $\mathrm{e}$ is $\mathrm{e}_{1}, \cdots, \mathrm{e}_{j}$, where $j>1, \eta(\mathrm{m}, \mathrm{e})$ is $\{\mathrm{m},\{\mathrm{e}\}$.

Lemma 17. For every formula $\mathrm{A}(\mathfrak{e}, \mathfrak{a}, \mathfrak{c}, \mathfrak{b})$ of Class $\Gamma$ containing exactly the free variables exhibited, there exists a numeral $m$ and a system $\mathfrak{T}$ such that the formula

$$
\operatorname{GyA}(e, \mathfrak{a}, \mathrm{c}, \mathfrak{y}) \equiv \operatorname{Hy}\left(\mathrm{P}_{k}(\eta(\boldsymbol{m}, \mathfrak{e}), \mathfrak{a}, \mathrm{y}) \&[\mathrm{y}]=\mathrm{c}\right)
$$

is provable in $\mathfrak{T}$.

For the proof we must consider cases according as $e$ and $\mathfrak{b}$ are respectively empty, contain 1 or more than 1 element.

We consider first the case in which both $\mathfrak{e}$ and $\mathfrak{b}$ contain 1 element.

There exists a normal recursive functor $f$, a numeral $\boldsymbol{m}$, and a system $\mathfrak{I}$ such that the following formulas are provable in $\mathfrak{T}$.

1. $A(e, \mathfrak{a}, c, d) \equiv f(e,\{a, c, d\})=0$. Lemmas 11,13 , and 14 . 
2. $f(e,\{a, c, d\})=0 \equiv \exists y(\psi(\{m, e,\{a, c, d\}, 0, y\})=0)$. Lemma 16 .

3. $\psi(\{m, e,\{a, c, d\}, 0, b\})=0 \equiv P_{k}(\{m, e\}, \mathfrak{a},\{c, d, b\}) \&(\{c, d, b\})_{0}$ $=$ c. Lemma 15, 8; Lemma 10, 10.7 .

4. Эx Эу $\psi(\{\boldsymbol{m}, \mathrm{e},\{\mathfrak{a}, \mathrm{c}, \mathrm{x}\}, 0, \mathrm{y}\})=0 \equiv \exists y\left(\mathrm{P}_{k}(\{\boldsymbol{m}, \mathrm{e}\}, \mathfrak{a}, \mathrm{y}) \&[\mathrm{y}]=\mathrm{c}\right)$. 3; Lemma 10, 10.7; Lemma 15, 8 and 10.

5. Эy $\mathrm{A}(\mathrm{e}, \mathfrak{a}, \mathrm{c}, \mathrm{y}) \equiv \exists \mathrm{y}\left(\mathrm{P}_{k}(\{m, \mathrm{e}\}, \mathfrak{a}, \mathrm{y}) \&[\mathrm{y}]=\mathrm{c}\right) .1,2,4$.

The remaining cases follow easily from this; of the cases for $\mathfrak{e}$ and $\mathfrak{b}$ not empty, we consider only the additional one in which both $\mathfrak{e}$ and $\mathfrak{b}$ have more than 1 element. that:

By the case just discussed, there exists a numeral $\boldsymbol{m}$ and a system $\mathfrak{T}$ such

1. $\exists y\left(\mathrm{~A}\left((\mathrm{e})_{0}, \cdots,(\mathrm{e})_{\delta(j)}, \mathfrak{a}, \mathrm{c},(\mathrm{y})_{0}, \cdots,(\mathrm{y})_{\delta(D)}\right) \& \mathrm{e}=\left\{(\mathrm{e})_{0}, \cdots,(\mathrm{e})_{\delta(j)}\right\}\right.$ $\left.\& \mathrm{y}=\left\{(\mathrm{y})_{0}, \cdots,(\mathrm{y})_{\boldsymbol{B}(l)}\right\}\right) \equiv \mathrm{By}\left(\mathrm{P}_{k}(\{\mathrm{~m}, \mathrm{e}\}, \mathfrak{a}, \mathrm{y}) \&[\mathrm{y}]=\mathrm{c}\right)$ is provable in $\mathfrak{T}:$ Utilizing Lemma 10, 10.7 we have the provability in $\mathfrak{I}$ of the formula.

2. $\exists \mathfrak{H} A(e, \mathfrak{a}, c, \mathfrak{y}) \equiv \mathcal{B} y\left(A\left(e, \mathfrak{a}, c,(y)_{0}, \cdots,(y)_{\delta(l)}\right) \& y=\left\{(y)_{0}, \cdots\right.\right.$, $\left.\left.(\mathrm{y})_{\delta(l)}\right\}\right)$.

Then substitution of $\{e\}$ for $e$ in 1 gives the desired result.

For the cases in which $\mathfrak{e}$ or $\mathfrak{b}$ are empty sequences, we replace, in the left member of $1, \mathrm{e}=\left\{(\mathrm{e})_{0}, \cdots,(\mathrm{e})_{\delta(j)}\right\}$ or $\mathrm{y}=\left\{(\mathrm{y})_{0}, \cdots,(\mathrm{y})_{\delta(l)}\right\}$ by $\mathrm{e}=\mathrm{e}$ or $\mathrm{y}=\mathrm{y}$, finally substituting 0 for $\mathrm{e}$.

Corollary. For every formula $\mathrm{A}(\mathfrak{e}, \mathfrak{a}, \mathfrak{b})$ of Class $\Gamma$ containing exactly the free variables exhibited, there exist numerals $\boldsymbol{m}$ and $\boldsymbol{p}$ and a system $\mathfrak{T}$ such that the formulas

$$
\operatorname{GyA}(e, \mathfrak{a}, \mathfrak{y}) \equiv \operatorname{HyP}_{k}(\eta(m, e), \mathfrak{a}, y),
$$

and

$$
\operatorname{HyA}(e, \mathfrak{a}, \mathfrak{y}) \equiv \forall y>P_{k}(\eta(p, e), \mathfrak{a}, \mathrm{y})
$$

are provable in $\mathfrak{T}$.

From the lemma we have the provability in some system $\mathfrak{I}_{1}$ of

$$
\operatorname{Gy}(\mathrm{A}(e, \mathfrak{a}, \mathfrak{y}) \& \mathrm{c}=\mathrm{c}) \equiv \mathrm{Hy}_{\mathrm{y}}\left(\mathrm{P}_{k}(\eta(m, e), \mathfrak{a}, \mathrm{y}) \&[\mathrm{y}]=\mathrm{c}\right),
$$

from which Formula (i) is easily obtained.

If the formula $A(e, \mathfrak{a}, \mathfrak{b})$ is of Class $\Gamma$ then $7 \mathrm{~A}(\mathfrak{e}, \mathfrak{a}, \mathfrak{b})$ is also of Class $\Gamma$. There is, then, a numeral $p$ and a system $\mathfrak{I}_{2}$ in which the formula

$$
\mathcal{H} \mathfrak{y} 7 \mathrm{~A}(e, \mathfrak{a}, \mathfrak{y}) \equiv \operatorname{HyP}_{k}(\eta(p, e), \mathfrak{a}, \mathrm{y})
$$

is provable. For any formula $\mathrm{C}(\mathrm{b})$ of $\mathfrak{T}_{2}, 7 \mathrm{GyC}(\mathrm{y}) \equiv \forall \mathrm{y} 7 \mathrm{C}(\mathrm{y})$ is provable in $\mathfrak{I}_{2}$, which gives for any $\mathrm{C}(\mathfrak{b})$,

Thus the formula 


$$
\forall \mathfrak{y} 77 \mathrm{~A}(\mathrm{e}, \mathfrak{a}, \mathfrak{y}) \equiv \forall \mathrm{y}>\mathrm{P}_{\mathfrak{h}}(\eta(p, e), \mathfrak{a}, \mathrm{y})
$$

is provable in some $\mathfrak{T}$, and applying Corollary 11.2, we obtain the provability of $77 \mathrm{~A}(\mathfrak{e}, \mathfrak{a}, \mathfrak{b}) \equiv \mathrm{A}(\mathfrak{e}, \mathfrak{a}, \mathfrak{b})$ in some $\mathfrak{T}_{3}$, which gives also Formula (ii) in any $\mathfrak{T}$ containing $\mathfrak{I}_{1}, \mathfrak{T}_{2}$, and $\mathfrak{I}_{3}$.

Lemma 18. For every formula $\mathrm{A}(e, \mathfrak{a}, \mathfrak{c}, \mathfrak{b})$ of Class $\Gamma$ containing exactly the free variables exhibited and such that the formula $\mathrm{B} \mathfrak{\eta A}\left(e, \mathfrak{a}, c_{1}, \mathfrak{h}\right)$ $\& \mathrm{G} \mathfrak{A A}\left(e, \mathfrak{a}, c_{2}, \mathfrak{y}\right) \supset c_{1}=c_{2}$ is provable in some system $\mathfrak{S}$, there exists a numeral $m$ and $a$ system $\mathfrak{T}$ such that the formula

$$
\mathfrak{H y A}(e, \mathfrak{a}, \mathrm{c}, \mathfrak{y}) \equiv \operatorname{Gy}\left(\mathrm{R}_{k}(\eta(m, e), \mathfrak{a}, \mathrm{y}) \&[\mathrm{y}]=\mathrm{c}\right)
$$

is provable in $\mathfrak{T}$.

There exists a numeral $\boldsymbol{m}$ and a system $\mathfrak{T}$ in which the following formulas are provable:

1. $\exists \mathfrak{y A}(e, \mathfrak{a}, \mathrm{c}, \mathfrak{y}) \equiv \exists y\left(\mathrm{P}_{k}(\eta(m, e), \mathfrak{a}, \mathrm{y}) \&[\mathrm{y}]=\mathrm{c}\right)$. Lemma 17.

2. $\mathcal{G y P}_{k}(\eta(m, e), \mathfrak{a}, \mathrm{y}) \supset \mathcal{B y R}_{k}(\eta(m, e), \mathfrak{a}, \mathrm{y})$. Lemma 15, 9; Lemma 12.

3. $\exists \mathfrak{A}(e, a, c, \mathfrak{y}) \supset \operatorname{\exists yR}_{k}(\eta(m, e), \mathfrak{a}, y) .1,2$.

4. $\mathrm{R}_{k}(\eta(m, e), a, d) \supset \mathrm{P}_{k}(\eta(m, e), \mathfrak{a}, \mathrm{d})$. Lemma 15, 9.

5. $\mathrm{R}_{k}(\eta(m, e), \mathfrak{a}, \mathrm{d}) \supset \exists \mathfrak{\sharp A}(e, \mathfrak{a},[\mathrm{d}], \mathfrak{y}) .4,1$.

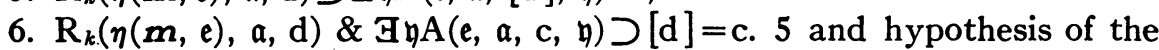
lemma.

7. $\exists \eta A(e, a, c, \mathfrak{y}) \supset \exists y\left(R_{k}(\eta(m, e), a, y) \&[y]=c\right) .3,6$.

8. $\mathrm{R}_{k}(\eta(m, e), \mathfrak{a}, \mathrm{d}) \&[\mathrm{~d}]=\mathrm{c} \supset \mathcal{\exists} \mathrm{yA}(\mathfrak{e}, \mathfrak{a}, \mathrm{c}, \mathfrak{y}) .5$.

9. $\exists y\left(R_{k}(\eta(m, e), a, y) \&[y]=c\right) \supset \exists \mathfrak{y A}(e, \mathfrak{a}, c, \mathfrak{y}) .8$.

Formulas 7 and 9 give us the desired result.

Corollary. For every formula $\mathrm{A}(\mathfrak{e}, \mathfrak{a}, \mathfrak{b})$ of Class $\Gamma$ containing exactly the free variables exhibited, there exist numerals $m$ and $p$ and a system $\mathfrak{T}$ such that the formulas

$$
\operatorname{GyA}(\mathfrak{e}, \mathfrak{a}, \mathfrak{y}) \equiv \operatorname{GyR}_{k}(\eta(\boldsymbol{m}, \mathfrak{e}), \mathfrak{a}, \mathrm{y})
$$

and

$$
\forall \mathfrak{y A}(e, \mathfrak{a}, \mathfrak{y}) \equiv \forall \mathrm{y}>\mathrm{R}_{k}(\eta(p, e), \mathfrak{a}, \mathrm{y})
$$

are provable in $\mathfrak{T}$.

From Lemma 15, 9, and Lemma 12 , we have the provability of $\operatorname{ByP}_{k}(\eta(m, e), \mathfrak{a}, \mathrm{y}) \equiv \mathrm{ByR}_{k}(\eta(\boldsymbol{m}, \mathrm{e}), \mathfrak{a}, \mathrm{y})$. This result and Formula (i) of the corollary to Lemma 17 give us Formula (i). Applying Formula 1 of the proof of that corollary to the same result, and using Formula (ii) of the corollary to Lemma 17, we obtain Formula (ii).

9. A reduction of any system $\subseteq$ to $S_{3}$. In the preceding section, to derive the properties of the formula $\mathrm{R}_{k}(\mathrm{e}, \mathfrak{a}, \mathrm{c})$ of $S_{3}$, we are obliged to consider the provability of formulas in systems which are extensions of $S_{3}$. We should 
like to be able, now, to show that formulas exhibiting the essential properties of $\mathrm{R}_{k}(\mathrm{e}, \mathfrak{a}, \mathrm{c})$ are provable in $S_{3}$. For example, we wish to show that, for any formula $\mathrm{A}(\mathfrak{a})$ of Class $\Gamma$ of $S_{3}$, there exists a numeral $\boldsymbol{m}$ such that $\mathrm{A}(\mathfrak{a})$ $\equiv \mathrm{BxR}_{k}(\mathrm{~m}, \mathfrak{a}, \mathrm{x})$ is provable in $S_{3}$.

We shall consider a transformation of the formulas of a system $\subseteq$ into formulas of $S_{3}$ which leaves the formulas of $S_{3}$ invariant. Let those type (c) functors which are neither type (b) nor type (a) functors be called proper type (c) functors; and let all those type (b) functors which are not type (a) functors be called proper type (b) functors. Then we define the transformation in three steps, successively eliminating those type (a) functors and proper type (b) and (c) functors which are not functors of $S_{\mathbf{3}}$.

Finally we obtain the result that a formula $\left(\left(\mathrm{A}^{\prime}\right)^{\prime \prime}\right)+$ is provable in $S_{8}$, if $A$ is provable in any $\mathfrak{S}$. It follows immediately from the invariance under this transformation of the formulas of $S_{3}$, that if any formula of $S_{3}$ is provable in any $\mathfrak{S}$, then it is also provable in $S_{3}$. We shall thus have shown an equivalence of any system $\subseteq$ containing $S_{3}$ to the system $S_{3}$.

Lemma 19. For every system $\mathfrak{S}$, there exists a system $\mathfrak{T}$ containing no proper type (c) functors except those of $S_{3}$ and such that, for every formula A provable in $\mathfrak{S}$, the formula $\mathrm{A}^{\prime}$ defined below is provable in $\mathfrak{T}$.

The formula $A^{\prime}$ is the result of replacing every proper type (c) functor $f$ except those of $S_{3}$ by the corresponding type (b) functor $\mathrm{f}^{\prime}$ which is defined in Lemma 13.

For each functor thus replaced, Lemma 13 determines a system $\mathfrak{I}^{\prime}$ corresponding to the system $\mathfrak{S}$. As axioms of the system $\mathfrak{I}$ for Lemma 19 , we choose the set-theoretic sum of the axioms of all those systems $\mathfrak{T}^{\prime}$ corresponding to the type (c) functors which are to be eliminated (compare the introductory portion of $\$ 3$ ), with the defining axioms of the same type (c) functors deleted. Likewise terms containing those type (c) functors are excluded from the terms of the new system (again under the conventions of $\S 3$ ).

For every axiom A of $\mathfrak{S}$ except the recursion equations deleted in forming the new system $\mathfrak{T}, \mathrm{A}^{\prime}$ will be an axiom of $\mathfrak{T}$. For each of the functors of $\mathfrak{S}$ which has been eliminated, we must show that the equations

$$
\begin{aligned}
\mathrm{f}^{\prime}(0, \mathfrak{a}) & =\mathrm{g}^{\prime}(\mathfrak{a}), \\
\mathrm{f}^{\prime}\left(\mathfrak{a}^{\prime}, \mathfrak{a}\right) & =\mathrm{h}^{\prime}\left(\mathrm{f}^{\prime}\left(\mathrm{t}_{1}^{\prime}(\mathrm{a}, \mathfrak{a}), \mathfrak{a}\right), \cdots, \mathrm{f}^{\prime}\left(\mathrm{t}_{k}^{\prime}(\mathrm{a}, \mathfrak{a}), \mathfrak{a}\right), \mathfrak{a}, \mathfrak{a}\right)
\end{aligned}
$$

are provable in $\mathfrak{T}$. This follows easily from the defining equations of $f^{\prime}$ in the proof of Lemma 13.

LEMMA 20. For every system $\mathfrak{T}$ containing no proper type (c) functors except those of $S_{3}$, there exists a system $\mathfrak{\Re}$ containing only type (a) functors and functors of $S_{3}$ such that, if $\mathrm{A}$ is provable in $\mathfrak{T}$, then $\mathrm{A}^{\prime \prime}$ defined below is provable in $\mathfrak{N}$.

We define $\mathrm{A}^{\prime \prime}$ inductively: 
If $\mathrm{A}$ is an elementary formula, $\mathrm{A}^{\prime \prime}$ is the result of replacing each term not of $S_{3}$ and of the form $\mathrm{f}\left(\mathrm{t}_{1}, \mathrm{t}_{2}, \cdots, \mathrm{t}_{n}\right)$ by $\mathrm{f}^{\prime}\left(\mathrm{t}_{1},\left\{\mathrm{t}_{2}, \cdots, \mathrm{t}_{n}\right\}\right), \mathrm{f}\left(\mathrm{t}_{1}, \mathrm{t}_{2}\right)$ by $f^{\prime}\left(t_{1}, t_{2}\right), f\left(t_{1}\right)$ by $f^{\prime}\left(t_{1}, 0\right)$, where $f^{\prime}$ is the functor defined in the proof of Lemma 14. The formula (A \& B ) ${ }^{\prime \prime}$ is $\mathrm{A}^{\prime \prime} \& \mathrm{~B}^{\prime \prime},(\mathrm{A} \vee \mathrm{B})$ " is $\mathrm{A}^{\prime \prime} \vee \mathrm{B}^{\prime \prime}$, $(\mathrm{A} \supset \mathrm{B})^{\prime \prime}$ is $\mathrm{A}^{\prime \prime} \supset \mathrm{B}^{\prime \prime},(7 \mathrm{~A})^{\prime \prime}$ is $7\left(\mathrm{~A}^{\prime \prime}\right),(\mathrm{GxA}(\mathrm{x}))^{\prime \prime}$ is $\exists \mathrm{G}\left(\mathrm{A}^{\prime \prime}(\mathrm{x})\right)$, and $(\operatorname{VxA}(\mathrm{x}))^{\prime \prime}$ is $\operatorname{Vx}\left(\mathrm{A}^{\prime \prime}(\mathrm{x})\right)$, where $(\mathrm{A}(\mathrm{a}))^{\prime \prime}$ is written $\mathrm{A}^{\prime \prime}(\mathrm{a})$ and $\mathrm{A}^{\prime \prime}(\mathrm{x})$ is the result of substituting a variable $x$ not occurring in $A(a)$ for $a$ in $A^{\prime \prime}(a)$.

The proof is like that of Lemma 19 . We have merely to show that formulas corresponding to type (b) defining axioms are provable in $\mathfrak{N}$, where $\mathfrak{N}$ is chosen, by applications of Lemma 14 which eliminate those proper type (b) functors not in $S_{3}$, in the same manner as that in which $\mathfrak{I}$ was chosen by applications of Lemma 13 which eliminate proper type (c) functors not in $S_{3}$.

To demonstrate an equivalence of any system, containing only normal recursive functors and the functors of $S_{3}$, with $S_{3}$, we define for each formula A of $\subseteq$ a formula $\mathrm{A}^{+}$of $S_{3}$.

In this definition and in the following discussion, we shall use $\psi(e, a, b, c)$ as an abbreviation for $\exists w \psi(\{e, a, b, c, w\})=0$. For this definition, let us also distinguish a functor position of $\mathrm{A}$ from a functor of A. For each occurrence of a functor not of $S_{3}$ there is a distinct functor position, and to each of these functor positions, proceeding from left to right, we assign a number, $1, \cdots, k$. We shall designate the functor which appears in the $i$ th functor position of $A$ by $f_{i}$. Thus for a given formula $A, f_{i}$ and $f_{j}$ may be the same functor although $i \neq j$. In addition let $\boldsymbol{n}_{\boldsymbol{i}}$ be the numeral specified by Lemma 16 for the functor $f_{i}$.

For the first part of the definition of $\mathrm{A}^{+}$, let $\mathrm{A}$ be the elementary formula $t_{01}=t_{02}$, and let the term which begins with the functor $f_{i}$ be $f_{i}\left(t_{i 1}, t_{i 2}\right)$.

Let $B_{1}$ be the expression which results on replacing the term $f_{1}\left(t_{11}, t_{12}\right)$ by $z_{1}$ in $A$. Note that, by the convention explained above, the choice of the term $f_{1}\left(t_{11}, t_{12}\right)$ is unique. Then let $A_{1}$ abbreviate the formula $\exists z_{1}\left(\psi\left(n_{1}, t_{11}, t_{12}, z_{1}\right)\right.$ $\left.\& B_{1}\right)$.

The first functor position of $\mathrm{A}_{1}$ containing a functor not of $S_{3}$ will contain the functor $f_{2}$. Now let $B_{2}$ abbreviate the result of replacing $f_{2}\left(t_{21}, t_{22}\right)$ by $z_{2}$ in $A_{1}$. Then $A_{2}$ is the formula $\exists z_{2}\left(\psi\left(n_{2}, t_{21}, t_{22}, z_{2}\right) \& B_{2}\right)$.

These operations are continued, generating a sequence of formulas $\mathrm{A}_{1}, \cdots, \mathrm{A}_{k}$ each containing one less occurrence of the functors of $\mathrm{A}$ which are not functors of $S_{3}$ than its predecessor. We terminate the process with the first formula which contains only functors of $S_{3}$; this formula $\mathrm{A}_{k}$ is $\mathrm{A}^{+}$.

For any elementary formula $\mathrm{A}$, the formula $\mathrm{A}^{+}$is of the form

$$
\text { 炙 } z_{k}\left(\psi\left(n_{k}, \xi_{k 1}, \xi_{k 2}, z_{k}\right) \& \cdots \& \text { 其 } z_{1}\left(\psi\left(n_{1}, \xi_{11}, \xi_{12}, z_{1}\right) \& \xi_{01}=\xi_{02}\right) \cdots\right) \text {, }
$$

where each $\xi_{i j}$ is the result of replacements on $t_{i j}$, and contains only functors of $S_{3}$, free variables, numerals and bound variables $z_{h}$, where $h>i$.

For example, suppose that $A$ is the formula $f_{1}\left(f_{2}(0, a), b\right)=f_{3}(c, d)$, then $A^{+}$ 
is the formula $\exists z_{3}\left(\psi\left(n_{3}, c, d, z_{3}\right) \& \exists z_{2}\left(\psi\left(n_{2}, 0, a, z_{2}\right) \& \exists z_{1}\left(\psi\left(n_{1}, z_{2}, b, z_{1}\right)\right.\right.\right.$ \& $\left.\left.z_{1}=z_{3}\right)\right)$ ).

For convenience, we write $\mathrm{A}^{+}$as $\Psi_{k} \cdots \Psi_{1} \xi_{01}=\xi_{02}$, where $\Psi_{i} \mathrm{C}$ is $\exists z_{i}\left(\psi\left(n_{i}, \xi_{i 1}, \xi_{i 2}, z_{i}\right) \& C\right)$. The operators $\Psi_{i}$ are, of course, always defined with respect to some specific formula $A$. We call $\Psi_{i}$ the companion operator of $f_{i}$ in $A$.

If either of the expressions $\xi_{i 1}$ or $\xi_{i 2}$ of $A^{+}$contains $z_{j}$, then the operator $\Psi_{j}$ is said to dominate the operator $\Psi_{i}$. This occurs exactly when $f_{j}$ is nested within $f_{i}$ in the formula $A$.

If neither of $\Psi_{i}$ and $\Psi_{j}$ dominates the other, then $\Psi_{i}$ and $\Psi_{j}$ are said to be independent.

If in a single context we wish to refer to the companion operators for more than one formula, we shall use the notations $\Psi_{i}^{\prime}, \Psi_{i}^{\prime \prime}$, and so on.

For a nonelementary formula $\mathrm{A}$, we define $\mathrm{A}^{+}$by replacing "/" by "+" in the second sentence of the definition of $A^{\prime \prime}$.

It is assumed, for any formula $A$, that the bound variables $z_{i}$ introduced in $\mathrm{A}^{+}$are chosen to be distinct from all the bound variables of $\mathrm{A}$.

LEMMA 21. For every axiom A which is a defining equation of a normal recursive functor $f$, the formula $\mathrm{A}^{+}$is provable in $S_{3}$.

The proof by induction on the number of functors upon which $f$ depends follows from Lemmas 10 and 15 with some rather tedious detail.

LEMMA 22. For every numeral $n$ which corresponds to a normal recursive functor (Lemma 16), the following formulas are provable in $S_{3}$ :

$$
\begin{gathered}
\text { Вy } \psi(n, a, b, y), \\
\psi(n, a, b, c) \& \psi(n, a, b, d) \supset c=d .
\end{gathered}
$$

The proof for (i) follows immediately from Lemma 21 . The proof of (ii) follows again by induction on the number of functors upon which the functor for $\boldsymbol{n}$ depends from Lemmas 10 and 15.

LEMMA 23. For any formula $\mathrm{R}(\mathfrak{a}, \mathrm{b})$ of $S_{3}$ containing exactly the free variables exhibited, and such that both $\mathrm{Gy} \mathrm{R}(\mathfrak{a}, \mathrm{y})$ and $\mathrm{R}\left(\mathrm{a}, \mathrm{b}_{1}\right) \& \mathrm{R}\left(\mathfrak{a}, \mathrm{b}_{2}\right) \supset \mathrm{b}_{1}=\mathrm{b}_{2}$ are provable in $S_{3}$, and for any formula $\mathrm{A}(\mathrm{b}), \mathrm{A}(\mathrm{a}, \mathrm{b})$, or any pair of formulas $\mathrm{A}(\mathrm{b})$ and $\mathrm{B}(\mathrm{b})$ of $S_{3}$, the following formulas are provable in $S_{3}$ : (In (5) and (6) it is understood that $x$ does not appear in $R(a, y)$. It is not excluded that some of the variables of $a$ may occur in $A(a), A(a, b)$, or $B(b)$; however, $a$ and $b$ are distinct from the variables $a$, cf. $\S 1$, first paragraph.) $\& \mathrm{~B}(\mathrm{y}))$.

(1) $\exists y(R(a, \quad y) \& A(y) \& B(y)) \equiv \exists y(R(a, \quad y) \& A(y)) \& \exists y(R(a, \quad y)$

(2) $\exists y(R(a, y) \&(A(y) \bigvee B(y))) \equiv \exists y(R(a, y) \& A(y)) \vee \exists y(R(a, y) \& B(y))$.

(3) $\exists y(R(a, y) \&(A(y) \supset B(y))) \equiv \exists y(R(a, y) \& A(y)) \supset \exists y(R(a, y) \& B(y))$. 
(4) $\exists y(R(a, y) \& 7 A(y)) \equiv 7 \exists y(R(a, y) \& A(y))$.

(5) $\exists y(R(a, y) \& \forall x A(x, y)) \equiv \forall x \exists y(R(a, y) \& A(x, y))$.

(6) $\operatorname{Gy}(\mathrm{R}(\mathfrak{a}, \mathrm{y}) \& \operatorname{\exists xA}(\mathrm{x}, \mathrm{y})) \equiv \exists x \operatorname{Bg}(\mathrm{R}(\mathfrak{a}, \mathrm{y}) \& \mathrm{~A}(\mathrm{x}, \mathrm{y}))$.

If in (1), (2) and (3), $A(y)$ is replaced by $A$ in the left member and $\operatorname{By}(R(\mathfrak{a}, y) \& A(y))$ is replaced by $A$, where $A$ does not contain $y$, every formula of the resulting form is provable in $S_{3}$, and likewise for replacement of $\mathrm{B}(\mathrm{y})$ by $\mathrm{B}$ not containing $\mathrm{y}$.

The proofs are by familiar properties of the predicate calculus.

LEMMA 24. If A is an elementary formula containing no functors except normal recursive ones and those of $S_{3}$ and $\mathrm{A}^{+} i s \Psi_{k} \cdots \Psi_{1} \xi_{01}=\xi_{02}$, and if $\Psi_{\nu_{k}} \ldots \Psi_{\nu_{1}}$ is a permutation of $\Psi_{k} \cdots \Psi_{1}$ in which no operator appears to the left of an operator which dominates it, the formula $\Psi_{\nu_{k}} \cdots \Psi_{\nu_{1}} \xi_{01}=\xi_{02} \equiv \mathrm{A}^{+}$is provable in $S_{3}$.

The lemma may be proved by induction on the number of interchanges of adjacent occurrences of independent companion operators which is required to effect the permutation $\Psi_{\eta_{k}} \ldots \Psi_{\eta_{1}}$. We have only to note the provability in $S_{3}$ of any formula of the form $\mathrm{Gx}(\mathrm{A}(\mathrm{x}) \& \mathrm{By}(\mathrm{B}(\mathrm{y}) \& \mathrm{C})) \equiv \mathrm{By}(\mathrm{B}(\mathrm{y})$ \& $\mathrm{Hx}(\mathrm{A}(\mathrm{x}) \& \mathrm{C})$ ), where $\mathrm{A}(\mathrm{x})$ does not contain $\mathrm{y}$ and $\mathrm{B}(\mathrm{y})$ does not contain $\mathrm{x}$.

Observe that the restriction on permutation of companion operators amounts to a limitation to those permutations which do not change the relative order of a pair of operators corresponding to initial functors of two terms, one contained in the other.

COROLlARY. If $\mathrm{A}(\mathrm{a})$ is an elementary formula containing no functors except normal recursive ones and those of $S_{3}$, and $\mathrm{A}^{+}(\mathrm{a})$ does not contain $\mathrm{y}$, then for every normal recursive functor $\mathrm{f}$ not a functor of $S_{3}$, there exists a numeral $n$ such that if $\mathrm{t}_{1}$ and $\mathrm{t}_{2}$ are terms of $S_{3}$, the formula $\left(\mathrm{A}\left(\mathrm{f}\left(\mathrm{t}_{1}, \mathrm{t}_{2}\right)\right)\right)^{+}$

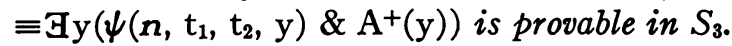

The occurrences of $f\left(t_{1}, t_{2}\right)$ in $A\left(f\left(t_{1}, t_{2}\right)\right)$ do not contain any functors except those of $S_{3}$. Thus the companion operators of these occurrences of $f$ are dominated by none of the other operators and, by the lemma, may be moved to the front of $\left(A\left(f\left(t_{1}, t_{2}\right)\right)\right)+$. Hence we have the provability of a formula of the form $\exists z_{1}\left(\psi\left(n, t_{1}, t_{2}, z_{1}\right) \& \cdots \& B z_{m}\left(\psi\left(n, t_{1}, t_{2}, z_{m}\right) \& G\left(z_{1}, \cdots, z_{m}\right)\right)\right.$ $\cdots \equiv\left(A\left(f\left(t_{1}, t_{2}\right)\right)\right)^{+}$, where $G(a, \cdots, a)$ is $A^{+}(a)$. From Lemma 22 , the formula $\psi(n, c, d, a) \& \psi(n, c, d, b) \supset a=b$ is provable in $S_{3}$, and this result enables us to obtain the provability of the desired formula.

LEMma 25. If $\mathrm{A}(\mathrm{a})$ is a formula containing no functors except normal recursive ones and those of $S_{3}$, and $\mathrm{A}^{+}(\mathrm{a})$ does not contain $\mathrm{y}$, then for every normal recursive functor $\mathrm{f}$ not a functor of $S_{3}$ there exists a numeral $\mathrm{n}$ such that, if $\mathrm{t}_{1}$ and $\mathrm{t}_{2}$ are terms of $S_{3}$, the formula $\left(\mathrm{A}\left(\mathrm{f}\left(\mathrm{t}_{1}, \mathrm{t}_{2}\right)\right)\right)+\supset \mathrm{Hy}\left(\psi\left(\mathrm{n}, \mathrm{t}_{1}, \mathrm{t}_{2}, \mathrm{y}\right) \& \mathrm{~A}^{+}(\mathrm{y})\right)$ is provable in $S_{3}$. 
The proof is by induction on the number of logical symbols contained in A(a) and follows from Lemmas 22 and 23 and the corollary to Lemma 24 .

COROLlARY 25.1. If neither the formula $\mathrm{A}(\mathrm{a})$ nor the term $\mathrm{t}$ contains any functors except normal recursive ones and those of $S_{3}$, and if $(\mathrm{t}=\mathrm{c})^{+}$is $\Psi_{l}^{\prime} \cdots \Psi_{1}^{\prime} \xi_{01}^{\prime}=\mathrm{c}$, then the formula $(\mathrm{A}(\mathrm{t}))^{+} \equiv \Psi_{l}^{\prime} \cdots \Psi_{1}^{\prime} \mathrm{A}^{+}\left(\xi_{01}^{\prime}\right)$ is provable in $S_{3}$.

The proof follows from the lemma by induction on the number of functors in $t$ which are not functors of $S_{\mathbf{3}}$.

For the proof of the following corollary, we note that the formula $\Psi_{i}^{\prime} \cdots \Psi_{1}^{\prime} \mathrm{A}^{+}\left(\xi_{01}^{\prime}\right)$ is of the form $\mathrm{H}_{l}\left(\mathrm{~F}_{l}\left(\mathrm{z}_{l}\right) \& \cdots \& \mathrm{Z}_{1}\left(\mathrm{~F}_{1}\left(\mathrm{z}_{1}\right) \& \mathrm{~A}^{+}\left(\xi_{01}^{\prime}\right)\right) \cdots \cdot\right)$, where $z_{l}, \cdots, z_{1}$ are bound variables which do not appear in $A^{+}(a)$.

CoRollaRy 25.2. If $\mathrm{A}(\mathrm{a})$ is a formula not containing $\mathrm{x}$ and $\mathrm{t}$ is a term, and if neither $\mathrm{A}(\mathrm{a})$ not $\mathrm{t}$ contains any functors except normal recursive ones and those of $S_{3}$, then the following formulas are provable in $S_{3}$ :

$$
\begin{aligned}
(\mathrm{A}(\mathrm{t}) & \supset \mathrm{GxA}(\mathrm{x}))^{+}, \\
(\mathrm{VxA}(\mathrm{x}) & \supset \mathrm{A}(\mathrm{t}))^{+} .
\end{aligned}
$$

The provability of a formula of the form (i) follows immediately from the preceding corollary by familiar rules of the predicate calculus upon recalling that (i) is of the form $(\mathrm{A}(\mathrm{t}))+\supset \exists \times \mathrm{A}^{+}(\mathrm{x})$.

For (ii) we apply the preceding corollary and Lemma 22, (i)

LeMma 26. If the formula $\mathrm{A}$ is provable in a system $\mathbb{S}$ which contains, in addition to functors of $S_{3}$, only normal recursive functors, then $\mathrm{A}^{+}$is provable in $S_{3}$.

For every axiom $A$ of $\mathfrak{S}$ of the form of Schemata $1 \mathrm{a}-13, \mathrm{~A}^{+}$is an axiom of $S_{3}$, except for 10 and 11 ; and the provability in $S_{3}$ of formulas of the forms of Schemata 10 and 11 follows from Corollary 25.2. For a defining equation A for a functor which is not in $S_{3}$, the provability of A+ in $S_{3}$ is given by Lemma 21.

If

$$
\frac{\mathrm{A}}{\mathrm{B}}
$$

is one of the rules of inference for $\mathfrak{S}$, then

$$
\frac{\mathrm{A}^{+}}{\mathrm{B}^{+}}
$$

is a rule of inference for $S_{3}$, and we establish the lemma by induction.

COROLLARY. Every formula of $S_{3}$ which is provable in any of the formal systems $\subseteq$ is also provable in $S_{3}$. 
10. An abbreviation for the representation of functions in $S_{3}$. Let A(b) be a formula, let $b$ be a free variable, let $y$ be a bound variable not appearing in $\mathrm{A}(\mathrm{b})$, let $\mathrm{e}$ be any term, and let $a$ abbreviate a sequence of terms $\mathrm{a}_{1}, \cdots, \mathrm{a}_{k}(k \geqq 1)$. Then we abbreviate $\left({ }^{11}\right)$ the formula $\exists \mathrm{y}\left(\mathrm{R}_{k}(\mathrm{e}, \mathfrak{a}, \mathrm{y}) \& \mathrm{~A}([\mathrm{y}])\right)$ by the expression $\mathrm{e}(\mathrm{A}(\mathrm{e}(\mathfrak{a})))$.

To avoid ambiguity in successive applications of the abbreviation, we must take care to note one restriction on writing $e(A(e(a)))$. If $A(b)$ contains expressions written in abbreviated form, then we write $e(A(e(\mathfrak{a})))$ only in case $A(b)$ contains no part written in the form ${ }_{e}(C)$ for the same $e$.

We may extend the application of this abbreviation in the following manner. Let $E\left(b_{1}, \cdots, b_{j}\right)$ be an abbreviation of the formula $A\left(b_{1}, \cdots, b_{j}\right)$, and suppose that the unabbreviated formula $B$ contains the expression $A\left(y_{1}, \cdots, y_{j}\right)$, where the variables $y_{1}, \cdots, y_{j}$ are bound by quantifiers in $B$ which are not in $A\left(b_{1}, \cdots, b_{j}\right)$. Then the formula $B$ may be abbreviated by replacing $\mathrm{A}\left(\mathrm{y}_{1}, \cdots, \mathrm{y}_{j}\right)$ by $\mathrm{E}\left(\mathrm{y}_{1}, \cdots, \mathrm{y}_{j}\right)$.

It is always understood that the bound variable which is suppressed in the abbreviation is one distinct from all the variables of the formula $A(b)$. If $\xi\left(\mathrm{A}\left(\xi\left(\zeta_{1}, \cdots, \zeta_{k}\right)\right)\right)$ is part of a formula $\mathrm{B}$, where $\xi$ and $\zeta_{1}, \cdots, \zeta_{k}$ may contain variables bound by quantifiers in $B$ but not in $A(b)$, then the bound variable which is suppressed is, in addition, distinct from all the other bound variables of $B$.

The abbreviation, which we shall call the functional abbreviation, will enable us to condense the statement of many results, particularly for formulas which express relations of composition among functions. For example consider the statement of the following result, which we shall later generalize as the corollary to Lemma 27.

Let $\mathrm{A}(\mathrm{b})$ be any formula of $S_{3}$ not containing the bound variables $\mathrm{z}$ and $\mathrm{w}$, let $\mathrm{t}(\mathrm{a})$ be any term of $S_{3}$ containing exactly the free variables e, a, let $\mathfrak{a}$ be $a_{1}, \cdots, a_{k}(k \geqq 1)$, let $\mathfrak{b}$ be $b_{1}, \cdots, b_{j}(j \geqq 1)$, and let $e$ be $e_{1}, \cdots, e_{l}(l \geqq 0)$. For any choice of the numeral $\boldsymbol{m}$ there exists a numeral $\boldsymbol{n}$ such that the following formula is provable in $S_{3}$ :

$$
\begin{aligned}
& \exists z\left(R_{k}(m, \mathfrak{a}, z) \& \operatorname{Hw}\left(\mathrm{R}_{j}(\mathrm{t}([\mathrm{z}]), \mathfrak{b}, \mathrm{w}) \& \mathrm{~A}([\mathrm{w}])\right)\right) \\
& \equiv \mathcal{G} w\left(\mathrm{R}_{k+j}(\eta(n, e), \mathfrak{a}, \mathfrak{b}, \mathrm{w}) \& \mathrm{~A}([\mathrm{w}])\right)
\end{aligned}
$$

(the explanation of the abbreviation $\eta(n, e)$ precedes Lemma 17). In the new notation this formula may be written:

$$
m(\mathfrak{t}(\boldsymbol{m}(\mathfrak{a}))(\mathrm{A}(\mathrm{t}(\boldsymbol{m}(\mathfrak{a}))(\mathfrak{b})))) \equiv_{\eta(n, e)}(\mathrm{A}(\eta(\boldsymbol{n}, \mathrm{e})(\mathfrak{a}, \mathfrak{b}))) .
$$

For the statement of the corollary below, we define inductively "quasiterm." A term is a quasi-term, and if $s$ is a quasi-term and $t$ is a sequence of

(11) Since adopting this treatment, it has come to the author's attention that it develops, for one case, a suggestion given in $[12, \S 2$, footnote 7$]$. The case is that in which the value of the function is defined for the arguments in question. 
quasi-terms, then $(\mathrm{s})(\mathrm{t})$ is a quasi-term.

A sequence of quasi-terms $\zeta_{1}, \cdots, \zeta_{k}$ is said to be a proper sequence of quasi-terms in case: for every $\zeta_{j}$ which contains a quasi-term of the form e $(t)$, there exists in the sequence a $\zeta_{i}$, where $i<j$, such that $\zeta_{i}$ is e.

Lemma 27. For every formula $\mathrm{A}(\mathfrak{e}, \mathfrak{a}, \mathfrak{b}, \mathrm{c})$ of Class $\Gamma$ containing exactly the free variables exhibited and such that $\exists \mathfrak{y A}\left(\mathfrak{e}, \mathfrak{a}, \mathfrak{y}, \mathrm{c}_{1}\right) \& \exists \mathfrak{\exists A}\left(\mathfrak{e}, \mathfrak{a}, \mathfrak{y}, \mathrm{c}_{2}\right) \supset \mathrm{c}_{1}$ $=\mathrm{c}_{2}$ is provable in $S_{3}$, there exists a numeral $n$ such that, for any formula $\mathrm{C}(\mathrm{c})$ of $S_{3}$ and for bound variables $z, \mathfrak{y}$ not appearing in $\mathrm{A}(\mathrm{e}, \mathfrak{a}, \mathfrak{b}, \mathrm{c})$ or $\mathrm{C}(\mathrm{c})$, the formula $\mathrm{Bz} \exists \mathfrak{y}(\mathrm{A}(\mathfrak{e}, \mathfrak{a}, \mathfrak{y}, \mathrm{z}) \& \mathrm{C}(\mathrm{z})) \equiv_{\eta(n, e)}(\mathrm{C}(\eta(n, \mathfrak{e})(\mathfrak{a})))$ is provable in $S_{3}$. (Note that $\mathrm{C}(\mathrm{c})$ may contain any of the variables $e, \mathfrak{a})$.

By Lemma 18, there exists a numeral $n$ such that the formula $\exists \mathfrak{y A}(e, \mathfrak{a}, \mathfrak{y}, \mathrm{c}) \equiv \exists y\left(\mathrm{R}_{k}(\eta(n, e), \mathfrak{a}, \mathrm{y}) \&[\mathrm{y}]=\mathrm{c}\right)$ is provable in some system $\mathfrak{S}$, and by the corollary to Lemma 26 , this formula is provable in $S_{3}$. Then by use of rules for the predicate calculus, the formula $\exists z(\exists \mathfrak{y ~} A(e, \mathfrak{a}, \mathfrak{y}, z) \& \mathrm{C}(z))$ $\equiv \mathrm{Bz}_{\mathrm{H}} \mathrm{y}\left(\mathrm{R}_{k}(\eta(\mathrm{n}, \mathrm{e}), \mathfrak{a}, \mathrm{y}) \&[\mathrm{y}]=\mathrm{z} \& \mathrm{C}(\mathrm{z})\right)$ is also provable in $S_{3}$. Finally by Schema 23 and rules of the predicate calculus, we have the provability of $\exists z \operatorname{Hy}(\mathrm{A}(\mathfrak{e}, \mathfrak{a}, \mathfrak{y}, z) \& \mathrm{C}(\mathrm{z})) \equiv \exists y\left(\mathrm{R}_{k}(\eta(\mathfrak{n}, \mathfrak{e}), \mathfrak{a}, \mathrm{y}) \& \mathrm{C}([\mathrm{y}])\right)$. Application of the abbreviation completes the demonstration.

Among the applications of Lemma 27, there are some in which the reader may be expected to supply the required formula $A(e, \mathfrak{a}, \mathfrak{b}, c)$. An important class of these cases is covered by the following proposition. If $\mathrm{t}$ is a term containing no functors except those of $S_{3}$, and containing exactly the variables $\mathfrak{e}, \mathfrak{a}$, then there exists a numeral $n$ such that $\mathrm{C}(\mathrm{t}) \equiv_{\eta(n, \mathrm{e})}(\mathrm{C}(\eta(n, e)(\mathfrak{a})))$ is provable in $S_{3}$. For proof, the $\mathrm{A}(\mathfrak{e}, \mathfrak{a}, \mathfrak{b}, c)$ of the lemma is simply $\mathrm{t}=\mathrm{c}$, with $\mathfrak{b}$ the empty sequence of variables. This proposition is generalized in the following corollary, of which it is the case for $p=0$.

CoROllaRY. If $\zeta_{1}, \cdots, \zeta_{p}, \zeta$ is a proper sequence of quasi-terms containing no functors except those of $S_{3}$ and containing exactly the free variables $\delta$, then for every arrangement of $\mathfrak{b}$ into a sequence $\mathfrak{e}, \mathfrak{a}$, where $\mathfrak{a}$ is not vacuous, there exists a numeral $n$ such that, for any formula $\mathrm{C}(\mathrm{c})$ of $S_{3}$, the formula ${ }_{{ }_{1}}\left(\cdots \zeta_{p}(\mathrm{C}(\zeta)) \cdots\right) \equiv_{\eta(n, \mathrm{e})}(\mathrm{C}(\eta(n, \mathrm{e})(\mathfrak{a})))$ is provable in $S_{3}$. (Note that $\mathrm{C}(\mathrm{c})$ may contain any of the variables $\delta$.)

If $\zeta_{1}, \cdots, \zeta_{p}, \zeta$ is a proper sequence of quasi-terms, then ${ }_{\zeta_{1}}\left(\cdots \zeta_{p}(\mathrm{C}(\zeta))\right.$ $\cdots)$ is of the form $\operatorname{By}_{1}\left(\mathrm{R}_{k_{1}}\left(\mathrm{~s}_{1}, \mathrm{t}_{1}, \mathrm{y}_{1}\right) \& \cdots \& \mathrm{By}_{p}\left(\mathrm{R}_{k_{p}}\left(\mathrm{~s}_{p}, \mathrm{t}_{p}, \mathrm{y}_{p}\right) \& \mathrm{C}\left(\left[\mathrm{y}_{p}\right]\right)\right.\right.$ $\cdots)$, where each $t_{i}$ is a sequence $t_{i 1}, \cdots, t_{i k_{i}}$, and each of $t_{i 1}, \cdots, t_{i k_{i}}, s_{i}$ is either a term or the result of replacement on certain free variables of a term by expressions $\left[\mathrm{y}_{j}\right]$, where $j<i$. Thus the following formula is provable in $S_{3}$ : $\zeta_{1}\left(\cdots \zeta_{p}(\mathrm{C}(\zeta)) \cdots\right) \equiv \mathrm{Hy}_{1} \cdots \mathrm{Hy}_{p}\left(\mathrm{R}_{k_{1}}\left(\mathrm{~s}_{1}, \mathrm{t}_{1}, \mathrm{y}_{1}\right) \& \cdots\right.$

$\left.\& \mathrm{R}_{k_{p}}\left(\mathrm{~s}_{p}, \mathrm{t}_{p}, \mathrm{y}_{p}\right) \& \mathrm{C}\left(\left[\mathrm{y}_{p}\right]\right)\right)$.

The right member of this formula is of the form $\mathbb{B} \mathfrak{H} \mathrm{Hy}_{p}\left(\mathrm{~F}\left(\mathfrak{e}, \mathfrak{a}, \mathfrak{y}, \mathrm{y}_{p}\right)\right.$ 
\& $\left.C\left(\left[y_{p}\right]\right)\right)$, where $\mathfrak{y}$ is $\mathrm{y}_{1}, \cdots, \mathrm{y}_{p-1}$. The formula $\mathrm{F}\left(\mathrm{e}, \mathfrak{a}, \mathrm{b}_{1}, \cdots, \mathrm{b}_{p}\right)$ is of Class $\Gamma$, and by the corollary to Lemma 15,5 the formula $\exists \mathfrak{y} F\left(e, \mathfrak{a}, \mathfrak{y}, \mathfrak{c}_{1}\right)$ $\& \exists \mathfrak{F}\left(\mathfrak{e}, \mathfrak{a}, \mathfrak{y}, c_{2}\right) \supset c_{1}=c_{2}$ is provable in $S_{3}$. Application of the lemma completes the demonstration.

Lemma 28. If $\mathrm{B}, \mathrm{A}(\mathrm{a}), \mathrm{B}(\mathrm{a}), \mathrm{A}(\mathrm{a}, \mathrm{b})$, and $\mathrm{B}(\mathrm{a}, \mathrm{b})$ are formulas of $S_{3}$, then the following formulas are provable in $S_{3}$ : (It is assumed that the subscript abbreviations appear only where exhibited and, for $1 \mathrm{~b}, 6,8-9 \mathrm{~b}$, that $\mathrm{A}(\mathrm{a})$, $\mathrm{B}(\mathrm{a}), \mathrm{A}(\mathrm{a}, \mathrm{b})$ and $\mathrm{B}(\mathrm{a}, \mathrm{b})$ do not contain the variable $\mathrm{x}$.

1a. $e(a=e(\mathfrak{a})) \supset\left(A(a) \supset_{e}(A(e(\mathfrak{a})))\right)$.

1b. $\mathrm{e}(\mathrm{A}(\mathrm{e}(\mathfrak{a}))) \& \forall \mathrm{x} B(\mathrm{x}) \supset_{\mathrm{e}}(\mathrm{B}(\mathrm{e}(\mathfrak{a})))$.

2. $\left.\left(\mathrm{e}(\mathrm{e}(\mathfrak{a})=\mathrm{c}) \supset_{\mathfrak{f}}(\mathrm{f}(\mathfrak{b})=\mathrm{c})\right) \supset_{(\mathrm{e}}(\mathrm{A}(\mathrm{e}(\mathfrak{a}))) \supset_{\mathfrak{f}}(\mathrm{A}(\mathrm{f}(\mathfrak{b})))\right)$.

3a. $\mathrm{e}(\mathrm{A}(\mathrm{e}(\mathfrak{a}))) \& \mathrm{e}_{\mathrm{e}}(\mathrm{B}(\mathrm{e}(\mathfrak{a}))) \equiv_{\mathrm{e}}(\mathrm{A}(\mathrm{e}(\mathfrak{a})) \& \mathrm{~B}(\mathrm{e}(\mathfrak{a})))$.

3b..$(A(e(a))) \& B \equiv_{e}(A(e(a)) \& B)$.

4a. $e(A(e(a))) \bigvee_{e}(B(e(a))) \equiv_{e}(A(e(a)) \bigvee B(e(\mathfrak{a})))$

4b. $e(A(e(a)) \bigvee B) \supset_{e}(A(e(a))) \vee B$.

5a. $(\mathrm{A}(\mathrm{e}(\mathfrak{a})) \supset \mathrm{B}(\mathrm{e}(\mathfrak{a}))) \supset\left(\mathrm{e}(\mathrm{A}(\mathrm{e}(\mathfrak{a}))) \supset_{\mathrm{e}}(\mathrm{B}(\mathrm{e}(\mathfrak{a})))\right)$.

5b. $\mathrm{e}(\mathrm{B} \supset \mathrm{A}(\mathrm{e}(\mathfrak{a}))) \supset(\mathrm{B} \supset \mathrm{e}(\mathrm{A}(\mathrm{e}(\mathfrak{a}))))$.

5c. $e(A(e(\mathfrak{a})) \supset B) \supset(e(A(e(\mathfrak{a}))) \supset B)$.

6. $\quad(\exists x A(e(\mathfrak{a}), x)) \equiv \exists x_{e}(A(e(\mathfrak{a}), x))$.

7. $\quad(\forall x A(e(a), x)) \equiv \forall x x_{e}(A(e(\mathfrak{a}), x))$.

8. $\quad(\mathrm{A}(\mathrm{e}(\mathfrak{a}))) \equiv \boldsymbol{G x}(\mathrm{e}(\mathrm{e}(\mathfrak{a})=\mathrm{x}) \& \mathrm{~A}(\mathrm{x}))$.

9a. $\forall x(A(x) \supset B(x)) \supset\left(e(A(e(a))) \supset_{e}(B(e(a)))\right)$.

9b. $\forall x(A(x) \supset B) \supset\left({ }_{e}(A(e(a))) \supset B\right)$.

The existence of proofs for the formulas $1 a-2,3 b-4 b, 5 b-6$, and $8-9 b$ follow immediately from rules for the predicate calculus, Schema 23, and Axiom 16.

For 3 a we apply the corollary to Lemma 15,5 , and the predicate calculus.

For $5 \mathrm{a}$, from the corollary to Lemma 15,5 , we have the provability of $\mathrm{R}_{k}(\mathrm{e}, \mathfrak{a}, \mathrm{d}) \&(\mathrm{~A}([\mathrm{~d}]) \supset \mathrm{B}([\mathrm{d}])) \& \mathrm{R}_{k}(\mathrm{e}, \mathfrak{a}, \mathrm{c}) \& \mathrm{~A}([\mathrm{c}]) \supset \exists \mathrm{G}\left(\mathrm{R}_{k}(\mathrm{e}, \mathfrak{a}, \mathrm{x})\right.$ $\& \mathrm{~B}([\mathrm{x}]))$. From this result the desired formula is easily obtained.

For 7 , from the corollary to Lemma 15,5 , we have the provability of $\mathrm{R}_{k}(\mathrm{e}, \mathrm{a}, \mathrm{a}) \& \mathrm{~A}([\mathrm{a}], \mathrm{c}) \supset\left(\mathrm{R}_{k}(\mathrm{e}, \mathfrak{a}, \mathrm{b}) \& \mathrm{~A}([\mathrm{~b}], \mathrm{d}) \supset \mathrm{R}_{k}(\mathrm{e}, \mathfrak{a}, \mathrm{a}) \& \mathrm{~A}([\mathrm{a}], \mathrm{d})\right)$; and thence, upon the introduction of quantifiers,

$$
\begin{aligned}
\operatorname{Hy}\left(\mathrm{R}_{k}(\mathrm{e}, \mathfrak{a}, \mathrm{y}) \& \mathrm{~A}([\mathrm{y}], \mathrm{c})\right) & \supset\left(\mathrm{\forall x} \mathrm{Hy}\left(\mathrm{R}_{k}(\mathrm{e}, \mathfrak{a}, \mathrm{y}) \& \mathrm{~A}([\mathrm{y}], \mathrm{x})\right)\right. \\
& \left.\supset \mathcal{G y}\left(\mathrm{R}_{k}(\mathrm{e}, \mathfrak{a}, \mathrm{y}) \& \operatorname{VxA}([\mathrm{y}], \mathrm{x})\right)\right) .
\end{aligned}
$$

The remainder of the proof for 7 is easily constructed.

Corollary. If $\mathrm{A}(\mathrm{a})$ is a formula of $S_{3}$, and if $\zeta_{1}, \cdots, \zeta_{p}, \theta_{1}$ and $\xi_{1}, \cdots, \xi_{q}, \theta_{2}$ are proper sequences of quasi-terms not containing the variable $\mathrm{c}$, and $p, q \geqq 0$, then the following formula is provable in $S_{3}$ : 


$$
\begin{aligned}
& \left(\zeta_{1}\left(\cdots \zeta_{p}\left(\theta_{1}=\mathrm{c}\right) \cdots\right) \supset \xi_{\xi_{1}}\left(\cdots_{\xi_{q}}\left(\theta_{2}=\mathrm{c}\right) \cdots\right)\right) \\
& \supset\left(\xi_{1}\left(\cdots_{\zeta_{p}}\left(\mathrm{~A}\left(\theta_{1}\right)\right) \cdots\right) \supset \xi_{\xi_{1}}\left(\cdots_{\xi_{q}}\left(\mathrm{~A}\left(\theta_{2}\right)\right) \cdots\right)\right) .
\end{aligned}
$$

(Note that $A(a)$ may contain any of the variables of $\zeta_{1}, \cdots, \zeta_{p}, \theta_{1}$, $\xi_{1}, \cdots, \xi_{q}, \theta_{2}$.)

This result follows from 2 by rules of the predicate calculus if we note that there exist numerals $\boldsymbol{m}$ and $\boldsymbol{n}$ such that the following are provable, $\mathrm{cf}$. Lemma 27, corollary:

$$
\begin{aligned}
\zeta_{1}\left(\cdots \zeta_{p}\left(\theta_{1}=\mathrm{c}\right) \cdots\right) & \equiv \boldsymbol{n}(\boldsymbol{n}(\mathfrak{a})=\mathrm{c}), \\
\zeta_{1}\left(\cdots \zeta_{p}\left(\mathrm{~A}\left(\theta_{1}\right)\right) \cdots\right) & \equiv \boldsymbol{n}(\mathrm{A}(\boldsymbol{n}(\mathfrak{a}))), \\
\xi_{1}\left(\cdots \xi_{q}\left(\theta_{2}=\mathrm{c}\right) \cdots\right) & \equiv \boldsymbol{m}(\boldsymbol{m}(\mathfrak{b})=\mathrm{c}), \\
\xi_{1}\left(\cdots \xi_{q}\left(\mathrm{~A}\left(\theta_{2}\right)\right) \cdots\right) & \equiv \boldsymbol{m}(\mathrm{A}(\boldsymbol{m}(\mathfrak{b}))),
\end{aligned}
$$

where $\mathfrak{a}$ is the list of free variables appearing in $\zeta_{1}, \cdots, \zeta_{p}, \theta_{1}$, and $\mathfrak{b}$ is the list of free variables in $\xi_{1}, \cdots, \xi_{q}, \theta_{2}$.

LeMma 29. For every natural number $k$, there exists a numeral $s_{k}$ such that, for any formulas $\mathrm{A}$ and $\mathrm{B}(\mathrm{a})$ of $S_{3}$, the formula

$$
\left(\mathrm{A} \supset \supset_{\mathrm{e}}(\mathrm{B}(\mathrm{e}(\mathfrak{a}, \mathrm{b})))\right) \equiv\left\{s_{k}, \mathrm{e}\right\}\left(\mathrm{A} \supset\{\mathrm{sk}, \mathrm{e}\}(\mathfrak{a})\left(\mathrm{B}\left(\left\{s_{k}, \mathrm{e}\right\}(\mathfrak{a})(\mathrm{b})\right)\right)\right),
$$

where $\mathfrak{a}$ is a sequence of terms $\mathrm{a}_{1}, \cdots, \mathrm{a}_{k}$, is provable in $S_{3}$. (Note that $\mathrm{A}$ and $B(a)$ may contain any of the variables $a, b$.)

By the corollary to Lemma 27 , there exists a numeral $n_{k}$ such that for any $\mathrm{B}(\mathrm{a})$ of $S_{3}$ the formula

$$
e(B(e(a, b))) \equiv\left\{n_{k},\{, a)\right\}\left(B\left(\left\{n_{k},\{e, a\}\right\}(b)\right)\right)
$$

is provable in $S_{3}$. Also by corollary to Lemma 27 there exists a numeral $s_{k}$ such that $\left\{n_{k},\{\mathrm{e}, \mathfrak{a}\}\right\}=\mathrm{c} \equiv{ }_{\left\{s_{k}, \mathrm{e}\right\}}\left(\left\{\boldsymbol{s}_{k}, \mathrm{e}\right\}(\mathfrak{a})=\mathrm{c}\right)$ is provable in $S_{3}$. From this, by substitution of $\left\{\boldsymbol{n}_{k},\{\mathrm{e}, \mathfrak{a}\}\right\}$ for $\mathrm{c}$, we obtain the provability of

$$
\left\{\boldsymbol{s}_{k}, \mathrm{e}\right\}\left(\left\{s_{k}, \mathrm{e}\right\}(\mathfrak{a})=\left\{\boldsymbol{n}_{k},\{\mathrm{e}, \mathfrak{a}\}\right\}\right) .
$$

From (i), we have the provability of

$$
\left(\mathrm{A} \supset \supset_{e}(\mathrm{~B}(\mathrm{e}(\mathfrak{a}, \mathrm{b})))\right) \equiv\left(\mathrm{A} \supset\left\{\boldsymbol{n}_{k},(e, a)\right\}\left(\mathrm{B}\left(\left\{\boldsymbol{n}_{k},\{\mathrm{e}, \mathfrak{a}\}\right\}(\mathrm{b})\right)\right)\right) \text {. }
$$

Applying Lemma 28, 1a to (ii) and (iii), we obtain the desired result.

For the statement of the following lemma we introduce a set of metamathematical operations somewhat like the operations $\Omega_{i}$ of $\S 1$. Let $a$ be a sequence of free variables $a_{1}, \cdots, a_{k}$, let $\mathfrak{x}$ be a sequence of bound variables $\mathrm{x}_{1}, \cdots, \mathrm{x}_{k}$, and let $\mathrm{C}_{\boldsymbol{v}_{1}}\left(\mathrm{a}_{1}, \cdots, \mathrm{a}_{v_{1}}\right), \cdots, \mathrm{C}_{\boldsymbol{p}_{p}}\left(\mathrm{a}_{1}, \cdots, \mathrm{a}_{v_{p}}\right)$, where $1 \leqq \nu_{j} \leqq k$, be a list of formulas of $S_{3}$ containing only those of the variables $a$ which are exhibited and none of the bound variables x. Let $A_{i}\left(a_{i}\right)$ contain the free vari- 
able indicated and not contain the bound variable $x_{i}$. Then if there exists a $\nu_{j}$ such that $\nu_{j}=i$, then $\Xi_{i} \mathrm{~A}_{i}\left(\mathrm{x}_{i}\right)$ is $\forall \mathrm{x}_{i}\left(\mathrm{C}_{i}\left(\mathrm{a}_{1}, \cdots, \mathrm{a}_{i-1}, \mathrm{x}_{i}\right) \supset \mathrm{A}_{i}\left(\mathrm{x}_{i}\right)\right)$. If there is no $\nu_{j}$ such that $\nu_{j}=i$, then $\Xi_{i} \mathrm{~A}_{i}\left(\mathrm{x}_{i}\right)$ is $\forall \mathrm{x}_{i} \mathrm{~A}_{i}\left(\mathrm{x}_{i}\right)$.

For natural numbers $1, \cdots, k-1$, let the numerals $s_{1}, \cdots, s_{k-1}$ be the ones specified in Lemma 29, and let e be any term of $S_{3}$. Then we let $\mathrm{f}_{k}$ abbreviate e, and for $1 \leqq j \leqq k-1$, we let $\mathrm{f}_{j}$ abbreviate $\left\{s_{j}, \mathrm{f}_{j+1}\right\}$. In addition let $f$ abbreviate $f_{1}$. (Note that when e is a numeral, then by Lemma 10 there is a numeral $f$ such that $f=f$ is provable in $S_{3}$. In cases in which Lemma 30 is employed to show the existence of a numeral, this fact will be used without further mention.)

Before stating the lemma we consider an example. Let $a$ be the sequence of free variables $a_{1}, a_{2}, a_{3}$. Let $C_{1}\left(a_{1}\right), C_{3}\left(a_{1}, a_{2}, a_{3}\right)$, and $C\left(a, a_{1}, a_{2}, a_{3}\right)$ be formulas containing exactly those variables of $\mathfrak{a}$ which are indicated, and not containing any of the bound variables $x_{1}, x_{2}, x_{3}$. Then the formula $\forall x_{1}\left(C_{1}\left(x_{1}\right)\right.$ $\left.\supset\left(\forall x_{2}\left(\forall x_{3}\left(C_{3}\left(x_{1}, x_{2}, x_{3}\right) \supset_{e}\left(C\left(e\left(x_{1}, x_{2}, x_{3}\right), x_{1}, x_{2}, x_{3}\right)\right)\right)\right)\right)\right)$ may be abbreviated by the expression (i) $\Xi_{1} \Xi_{2} \Xi_{3} \mathrm{e}\left(\mathrm{C}\left(\mathrm{e}\left(\mathrm{x}_{1}, \mathrm{x}_{2}, \mathrm{x}_{3}\right), \mathrm{x}_{1}, \mathrm{x}_{2}, \mathrm{x}_{3}\right)\right)$. The formula $\forall x_{1}\left(C_{1}\left(x_{1}\right) \supset_{f}\left(\forall x_{2} f\left(x_{1}\right)\left(\forall x_{3}\left(C_{3}\left(x_{1}, x_{2}, x_{3}\right) \supset_{f\left(x_{1}\right)\left(x_{2}\right)}\left(C\left(f\left(x_{1}\right)\left(x_{2}\right)\left(x_{3}\right), x_{1}, x_{2}, x_{3}\right)\right)\right)\right)\right)\right)$ may be abbreviated by (ii) $\Xi_{1 \mathrm{f}}\left(\boldsymbol{\Xi}_{2 \mathrm{f}\left(\mathrm{x}_{1}\right)}\left(\boldsymbol{\Xi}_{3 \mathrm{f}\left(\mathrm{x}_{1}\right)\left(\mathrm{x}_{2}\right)}\left(\mathrm{C}\left(\mathrm{f}\left(\mathrm{x}_{1}\right)\left(\mathrm{x}_{2}\right)\left(\mathrm{x}_{3}\right), \mathrm{x}_{1}, \mathrm{x}_{2}, \mathrm{x}_{3}\right)\right)\right)\right.$ ). The equivalence of Formulas (i) and (ii) will be seen to be an example for the following lemma.

In successive applications of the functional abbreviation, we further abbreviate by writing for an expression of the form $\left(\cdots\left(\left(\mathrm{e}\left(\mathfrak{a}_{1}\right)\right)\left(\mathfrak{a}_{2}\right)\right) \cdots\right.$ $\left.\left(\mathfrak{a}_{j-1}\right)\right)\left(\mathfrak{a}_{j}\right)$ where $\mathrm{e}$ is a term and $\mathfrak{a}_{i}, 1 \leqq i \leqq j$, is a sequence of terms $\mathrm{a}_{\mathfrak{i}_{1}}, \cdots, \mathrm{a}_{\boldsymbol{i}_{i}}$, the expression $\mathrm{e}\left(\mathfrak{a}_{1}\right)\left(\mathfrak{a}_{2}\right) \cdots\left(\mathfrak{a}_{j-1}\right)\left(\mathfrak{a}_{j}\right)$.

LeMma 30. For every formula $\mathrm{C}(\mathrm{a}, \mathfrak{a})$ of $S_{3}$, not containing bound variables $\mathrm{x}_{1}, \cdots, \mathrm{x}_{k}$, the following formula is provable in $S_{3}$ :

$\Xi_{1} \Xi_{2} \ldots \Xi_{k_{\mathrm{e}}}(\mathrm{C}(\mathrm{e}(\mathfrak{x}), \mathfrak{x}))$

$$
\equiv \Xi_{1 \mathrm{f}}\left(\boldsymbol{\Xi}_{2 \mathrm{f}\left(\mathrm{x}_{1}\right)}\left(\cdots\left(\boldsymbol{\Xi}_{k \mathrm{f}\left(\mathrm{x}_{1}\right) \cdots\left(\mathrm{x}_{k-1}\right)}\left(\mathrm{C}\left(\mathrm{f}\left(\mathrm{x}_{1}\right) \cdots\left(\mathrm{x}_{k}\right), \mathfrak{x}\right)\right)\right) \cdots\right)\right) .
$$

If $\mathfrak{b}$ is a sequence of free variables $b_{1}, \cdots, b_{i}$ and $A(b)$ and $B(a, b)$ are formulas not containing the bound variable $\mathrm{x}$ but possibly some of the variables $\mathfrak{b}$, then the following formulas are provable in $S_{3}$ :

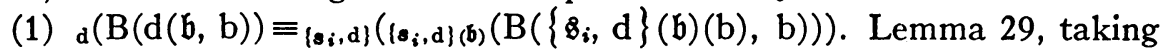
as $\mathrm{A}$ any provable formula.

(2) $\forall x_{d}(B(d(\mathfrak{b}, x), x)) \equiv_{\left\{s_{i}, d\right\}}\left(\forall x_{\left\{s_{i}, d\right\}(b)}\left(B\left(\left\{s_{i}, d\right\}(b)(x), x\right)\right)\right)$. Lemma $28,7$.

(3) $\forall \mathrm{x}\left(\mathrm{A}(\mathrm{x}) \supset_{\mathrm{d}}(\mathrm{B}(\mathrm{d}(\mathfrak{b}, \mathrm{x}), \mathrm{x}))\right) \equiv_{\left\{s_{i}, \mathrm{~d}\right\}}\left(\forall \mathrm{x}\left(\mathrm{A}(\mathrm{x}) \supset_{\left\{s_{i}, \mathrm{~d}\right\}(\mathfrak{b})}\left(\mathrm{B}\left(\left\{s_{i}, \mathrm{~d}\right\}(\mathfrak{b})(\mathrm{x})\right.\right.\right.\right.$, $x)))$ ). Lemma 29, Lemma 28, 7.

For each natural number $j, 1 \leqq j \leqq k$, let $a_{j}$ abbreviate the sequence $\mathrm{a}_{1}, \cdots, \mathrm{a}_{j}$ and let $\mathfrak{t}_{j}$ abbreviate the sequence $\mathrm{x}_{j+1}, \cdots, \mathrm{x}_{k}$. Then we shall use induction on $j$ decreasing from $k$ to 1 to show that the following formula is provable in $S_{3}$ for each of these values of $j$ : 
(4)

$$
\begin{aligned}
& \Xi_{j+1} \Xi_{j+2} \cdots \Xi_{k e}\left(C\left(e\left(\mathfrak{a}_{j}, \mathfrak{f}_{j}\right), \mathfrak{a}_{j}, \mathfrak{f}_{j}\right)\right)
\end{aligned}
$$

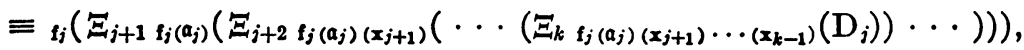

where $D_{j}$ is the expression $\mathrm{C}\left(\mathfrak{f}_{j}\left(\mathfrak{a}_{j}\right)\left(\mathrm{x}_{j+1}\right) \cdots\left(\mathrm{x}_{k-1}\right)\left(\mathrm{x}_{k}\right), \mathfrak{a}_{j}, \mathfrak{f}_{j}\right)$.

For the basis, the case in which $j=k$, by the definition of $\mathrm{f}_{k}$, e $(\mathrm{C}(\mathrm{e}(\mathfrak{a}), \mathfrak{a}))$ $\equiv_{\mathfrak{f}_{k}}\left(\mathrm{C}\left(\mathrm{f}_{k}(\mathfrak{a}), \mathfrak{a}\right)\right)$ is provable in $S_{3}$.

Then assuming the provability in $S_{8}$ of Formula (4) for a given value of $j>1$, and applying the appropriate one of (2) and (3), taking $i$ as $j-1, \mathrm{~d}$ as $f_{j}, \mathfrak{b}, b$ as $\mathfrak{a}_{j}, x$ as $x_{j}$, d $(B(d(\mathfrak{b}, b), b)$ ) as the right member of (4), and in the case that (3) is applied, A(b) as $C_{j}\left(\mathfrak{a}_{j}\right)$, we obtain a formula of the form of (4) with $j$ replaced by $j-1$. This completes the induction proof of (4).

For $j=1$ the formula of the form of (4) is

$$
\begin{aligned}
& \Xi_{2} \ldots \Xi_{k \mathrm{e}}\left(\mathrm{C}\left(\mathrm{e}\left(\mathrm{a}_{1}, \mathfrak{l}_{1}\right), \mathrm{a}_{1}, \mathfrak{l}_{1}\right)\right) \\
& \equiv_{\mathrm{f}}\left(\Xi_{2 f\left(\mathrm{a}_{1}\right)}\left(\cdots\left(\Xi_{k f\left(\mathrm{a}_{1}\right)\left(\mathrm{x}_{2}\right) \cdots\left(\mathrm{x}_{k-1}\right)}\left(\mathrm{C}\left(\mathrm{f}\left(\mathrm{a}_{1}\right)\left(\mathrm{x}_{2}\right) \cdots\left(\mathrm{x}_{k}\right), \mathrm{a}_{1}, \mathfrak{f}_{1}\right)\right)\right) \cdots\right)\right) \text {. }
\end{aligned}
$$

From this we easily obtain the desired result.

\section{PART IV}

In Part IV we consider the problem of formalizing the results of Part I and some additional results as well. The application of these formal results has already been discussed in Kleene's work. Assuming the simple consistency of the intuitionistic system $S_{3}$, Corollary 4.1 and the corollary to Theorem 5 will serve to establish the simple consistency of a system which is an extension of the intuitionistic systems $\mathbb{S}$ and which diverges from the classical systems $\mathfrak{C}[15, \S 14]$. Theorem 6 and either Theorem 7.1 or Theorem 7.2 are the basis of a demonstration of the unprovability of certain formulas of the intuitionistic predicate calculus, as well as of the incompleteness of the systems S with respect to realizability $[15, \S \S 15-16]$.

11. Formal representation of the realizability predicate. Since the predicate $T_{k}\left(e, a_{1}, \cdots, a_{k}, b\right)$ and the function $U(b)$ are primitive recursive, there exists a system $\subseteq$ containing formulas which have the form of recursion equations for the functions $\tau_{k}\left(e, a_{1}, \cdots, a_{k}, b\right)$ and $U(b)$, where $\tau_{k}\left(e, a_{1}, \cdots, a_{k}, b\right)=0$ if and only if $T_{k}\left(e, a_{1}, \cdots, a_{k}, b\right)$, and such that, by Lemma 18 and its corollary, there exist numerals $t_{k}, u$, and $\boldsymbol{v}_{k}$ for which the following formulas, where $\mathfrak{a}$ is $a_{1}, \cdots, a_{k}$, are provable in $\mathfrak{S}$ :

1. $\tau_{k}(\mathrm{e}, \mathfrak{a}, \mathrm{b})=0 \equiv \exists \mathrm{Bx} \mathrm{R}_{k+1}\left(\left\{t_{k}, \mathrm{e}\right\}, \mathfrak{a}, \mathrm{b}, \mathrm{x}\right)$.

2. $U(b)=c \equiv \exists x\left(R_{1}(u, b, x) \&[x]=c\right)$.

3. $\exists x\left(\tau_{k}(e, a, x)=0 \& U(x)=c\right) \equiv \exists x\left(R_{k}\left(\left\{v_{k}, e\right\}, a, x\right) \&[x]=c\right)$.

Then, by Lemmas 19 and 20 and Lemma 26, there exist formulas of $S_{8}$, $\mathrm{T}_{k}(\mathrm{e}, \mathfrak{a}, \mathrm{b})$ and $\mathrm{U}(\mathrm{b}, \mathrm{c})$, containing exactly the free variables exhibited, and such that the following formulas are provable in $S_{3}$ :

4. $\mathrm{T}_{k}(\mathrm{e}, \mathfrak{a}, \mathrm{b}) \equiv \exists \mathrm{x} \mathrm{R}_{k+1}\left(\left\{\boldsymbol{t}_{k}, \mathrm{e}\right\}, \mathfrak{a}, \mathrm{b}, \mathrm{x}\right)$.

5. $\mathrm{U}(\mathrm{b}, \mathrm{c}) \equiv \exists \mathrm{Gx}\left(\mathrm{R}_{1}(u, b, \mathrm{x}) \&[\mathrm{x}]=\mathrm{c}\right)$. 
6. $\exists x\left(T_{k}(e, \mathfrak{a}, \mathrm{x}) \& \mathrm{U}(\mathrm{x}, \mathrm{c})\right) \equiv \exists \mathrm{x}\left(\mathrm{R}_{k}\left(\left\{\mathrm{v}_{k}, \mathrm{e}\right\}, \mathfrak{a}, \mathrm{x}\right) \&[\mathrm{x}]=\mathrm{c}\right)$.

We are now in a position, using Formula 6 and results of Kleene $[13, \S \S 4$, $7]$, to assert the following: For every partial recursive function $\phi\left(x_{1}, \cdots, x_{k}\right)$, there exists a numeral $m$ such that if $\phi\left(n_{1}, \cdots, n_{k}\right)=n$, the formula $\exists \mathrm{Jx}\left(\mathrm{R}_{k}\left(\boldsymbol{m}, \boldsymbol{n}_{1}, \cdots, \boldsymbol{n}_{k}, \mathrm{x}\right) \&[\mathrm{x}]=\mathbf{n}\right)$ is provable in $S_{3}$.

Let us return to the definition of a formula $a \AA A$ for every formula $A$, this time with respect to the system $S_{3}$. We may employ the definition given by Kleene $[15, \S 12]$, using the present formulas $T_{1}(e, a, b)$ and $U(b, c)$, and, as in $\S 2$, replacing each part of the form $U(b) \circledast A$ by $\exists y(U(b, y) \& y @ A)$. For each formula $\mathrm{A}$, let the formula $\mathrm{a} \circledast \mathrm{A}$ thus obtained be designated by $\mathrm{a} \circledast_{1} \mathrm{~A}$.

However, utilizing the abbreviation of $\$ 10$, we may establish an equivalent definition which is more convenient for the considerations which we are about to undertake.

Hence, we define inductively for each formula $\mathrm{A}$ of $S_{3}$ a formula a $\AA_{2} \mathrm{~A}$ by the following clauses, in which $\mathrm{F}$ is any elementary formula of $S_{3}$, and A, $\mathrm{B}$ and $\mathrm{A}(\mathrm{b})$ are any formulas of $S_{3}$. In work following this definition we shall refer to the separate clauses as D1, and so on.

1. $\mathrm{e} \AA_{2} \mathrm{~F}$ is $\mathrm{e}=0 \& \mathrm{~F}$.

2. $\mathrm{e} \AA_{2}(\mathrm{~A} \& \mathrm{~B})$ is $(\mathrm{e})_{0} \AA_{2} \mathrm{~A} \&(\mathrm{e})_{1} \AA_{2} \mathrm{~B}$.

3. $\mathrm{e} \AA_{2}(\mathrm{~A} \vee \mathrm{B})$ is $\left((\mathrm{e})_{0}=0 \&(\mathrm{e})_{1} \AA_{2} \mathrm{~A}\right) \vee\left((\mathrm{e})_{0}=1 \&(\mathrm{e})_{1} \AA_{2} \mathrm{~B}\right)$.

4. $e \Theta_{2}(A \supset B)$ is $\forall x\left(x \AA_{2} A \supset_{0}\left(e(x) \circledast_{2} B\right)\right)$.

5. $\mathrm{e} \AA_{2}>\mathrm{A}$ is $\forall \mathrm{x}\left(\mathrm{x} \AA_{2} \mathrm{~A} \supset \mathrm{e}\left(\mathrm{e}(\mathrm{x}) \Theta_{2} 1=0\right)\right)$.

6. $\mathrm{e} \Theta_{2} \mathrm{Ax} A(\mathrm{x})$ is $(\mathrm{e})_{1} \AA_{2} \mathrm{~A}\left((\mathrm{e})_{0}\right)$.

7. $\mathrm{e} \Theta_{2} \forall x A(x)$ is $\forall x{ }_{0}\left(e(x) \circledast_{2} A(x)\right)$.

We have the following relation between the formulas representing the realizability predicate.

THEOREM 3. For every formula $\mathrm{A}$ of $S_{3}$ the formula $\mathrm{Gx}\left(\mathrm{x} \circledast{ }_{1} \mathrm{~A}\right) \equiv \exists \mathrm{x}\left(\mathrm{x} \otimes_{2} \mathrm{~A}\right)$ is provable in $S_{3}$.

The proof is a straightforward one by induction on the number of logical symbols in A.

In the remainder of the present work, we shall let $a @ A$ designate the formula $\mathrm{a} \AA_{2} \mathrm{~A}$.

12. Formal properties of realizability in $S_{3}$. In Part III, when we defined $S_{3}$, we might have included additional axioms in the form of recursion equations for functors of any of the types (a), (b), or (c). All of the results of Part III and \$11 would have held for the system thus formed. It may be observed that the following results of Part IV will also hold for any such system.

Lemma 31. If A is an axiom of $S_{3}$, then there exists a term $\mathrm{t}$ of $S_{3}$, such that the formula $\mathrm{t} \circledast \mathrm{A}$ is provable in $S_{3}$. (Note that a term $\mathrm{t}$ satisfying the conditions of the lemma may be chosen to contain no free variables besides those of A.) 
The defining axioms for recursive functions and Axiom 16 may be dismissed immediately, since, for each of these axioms $A$, the formula $0 \AA A$ is provable in $S_{3}$, cf. D1. There remain axioms of Schemata 1a, 1b, 3-8, 10, 11, 13, and 23, and Axioms 14, 15, 17, and 18, cf. $\$ 1$. We consider the cases for these by the numbers listed. To complete certain of the cases we apply Lemma 30 to the starred formulas.

$1 \mathrm{a}$.

1. $a ® A \supset(\{a, b\})_{0} \circledast A$. Schema 23 ; Lemma $10,10.7$.

2. $a \circledast A \supset \exists x\left((\{a, b\})_{0}=x \& x \circledast A\right) .1$.

3. $\mathrm{a} @ \mathrm{~A} \supset_{n}(n(\mathrm{a}, \mathrm{b}) \circledast \mathrm{A}) .2 ;$ Lemma 27.

4.* $\forall x(x ® A \supset \forall y(y \circledast B \supset n(n(x, y) \circledast A))) .3$.

The application of Lemma 30 assures the existence of a numeral $m$ such that the formula $\forall x\left(x \circledast A \supset_{m}\left(\forall y\left(y \circledast B \supset_{m(x)}(m(x)(y) ® A)\right)\right)\right)$ is provable in $S_{3}$. Upon two applications of D4, we check that this is the desired formula for an axiom of the form of $1 \mathrm{a}$.

$1 b$.

Let $F(e)$ abbreviate the formula $\forall x\left(x \circledast A \supset_{0}\left(\forall y\left(y \circledast B \supset_{e(x)}(e(x)(y)\right.\right.\right.$ $(B)))$ ), let $G(d)$ abbreviate $\forall x\left(x @ A \supset_{d}(d(x) \circledast B)\right)$, and let $H$ abbreviate $\mathrm{F}(\mathrm{e}) \& \mathrm{G}(\mathrm{d}) \& \mathrm{a} \circledast \mathrm{A}$.

1. $\mathrm{H}_{\mathrm{d}}(\mathrm{d}(\mathrm{a}) \circledast \mathrm{B}) \&_{\theta}\left(\mathrm{Vy}\left(\mathrm{y} \circledast \mathrm{B} \supset_{e(\mathrm{a})}(\mathrm{e}(\mathrm{a})(\mathrm{y}) \circledast \mathrm{C})\right)\right)$. Definition of $\mathrm{H}$, $\mathrm{G}(\mathrm{d})$, and $\mathrm{F}(\mathrm{e})$.

2. $H \supset_{\mathrm{d}}(\mathrm{d}(\mathrm{a}) \circledast \mathrm{B}) \& \mathrm{Vy} \cdot\left(\mathrm{y} \circledast \mathrm{B} \supset_{\mathrm{e}(\mathrm{a})}(\mathrm{e}(\mathrm{a})(\mathrm{y}) \circledast \mathrm{C})\right) .1$; Lemma $28,7$.

3. $H \supset_{d}(d(a) @ B) \&_{d}\left({ }_{e}\left(d(a) \circledast B \supset_{e(a)}(e(a)(d(a)) @ C)\right)\right)$. 2; Lemma 28 , $1 \mathrm{~b}$.

4. $H \supset_{d}(d(a) \circledast B) \&\left({ }_{d}(d(a) \circledast B) \supset_{d}\left({ }_{e}(e(a)(e(a)(d(a)) @ C))\right)\right)$. 3; Lemma $28,5 a, 5 b$.

5. $\mathrm{H} \supset_{\mathrm{d}}(\mathrm{e}(\mathrm{e}(\mathrm{a})(\mathrm{e}(\mathrm{a})(\mathrm{d}(\mathrm{a})) \AA \mathrm{C}))) .4$.

6. $\mathrm{H} \supset_{n}(n(e, d, a) \circledast C) .5$; corollary to Lemma 27.

7.* $\mathrm{Vw}\left(\mathrm{F}(\mathrm{w}) \supset \mathrm{Vz}\left(\mathrm{G}(\mathrm{z}) \supset \mathrm{Vx}\left(\mathrm{x} \AA \mathrm{A} \supset_{n}(n(\mathrm{w}, \mathrm{z}, \mathrm{x}) \AA \mathrm{C})\right)\right)\right)$. 6; and definition of $\mathrm{H}$.

\section{3.}

1. $a \circledast A \& b \circledast B \supset\{a, b\} \circledast(A \& B) . D 2 ;$ Lemma $10,10.7$.

2. $\mathrm{a} \circledast \mathrm{A} \& \mathrm{~b} \circledast \mathrm{B} \supset_{n}(n(\mathrm{a}, \mathrm{b}) \circledast(\mathrm{A} \& \mathrm{~B})) .1$; Lemma 27 applied as in case $1 \mathrm{a}$.

3.* $\forall x\left(x @ A \supset \forall y\left(y @ B \supset_{n}(n(x, y) \circledast(A \& B))\right)\right) .2$.

4 a.

1. $\mathrm{c}(\mathrm{A} \& \mathrm{~A} \mathrm{~B}) \supset(\mathrm{c})_{0}(\mathrm{~A}$. D2; Lemma 10, 10.7.

2. $\quad\left(@(A \& B) \supset_{n}(n(c) \circledast A) .1\right.$; Lemma 27.

3. $\forall x\left(x ®(A \& B) \supset_{n}(n(x) \circledast A)\right) .2$.

$4 b$.

Similar to $4 a$. 
$5 a$.

1. $c ® A \supset(\{0, c\})_{0}=0 \&(\{0, c\})_{1} \circledast A$. Lemma 10. 10.7.

2. $c ® A \supset\{0, c\} \circledast(A \vee B) .1 ; D 3$.

3. $\mathrm{c} \circledast \mathrm{A} \supset_{n}(n(\mathrm{c}) \circledast(\mathrm{A} \vee \mathrm{B})) .2 ;$ Lemma 27.

4. $\forall x(x \circledast A \supset n(n(x) \circledast(A \vee B))) .3$.

$5 \mathrm{~b}$.

Similar to 5 a.

6.

Let the following abbreviations be made: $P(c)$ for $\forall x\left(x \circledast A \supset_{0}(c(x) \circledast C)\right)$, $Q(d)$ for $\forall y\left(y \circledast B \supset_{d}(d(y) \circledast C)\right), \quad R(e)$ for $\left((e)_{0}=0 \quad \&(e)_{1} \AA A\right) \vee\left((e)_{0}=1\right.$ $\&(\mathrm{e})_{1}(\mathrm{~B})$, and $\mathrm{H}$ for $\mathrm{P}(\mathrm{c}) \& \mathrm{Q}(\mathrm{d}) \& \mathrm{R}(\mathrm{e})$.

1. $\left.\left.\mathrm{H} \supset\left((\mathrm{e})_{0}=0 \& \&_{0}\left(\mathrm{c}(\mathrm{e})_{1}\right) \circledast \mathrm{C}\right)\right)^{\prime} \bigvee\left((\mathrm{e})_{0}=1 \& \mathrm{~d}_{\mathrm{d}}\left(\mathrm{d}(\mathrm{e})_{1}\right) \circledast \mathrm{C}\right)\right)$. Definition of $\mathrm{H}, \mathrm{P}(\mathrm{c}), \mathrm{Q}(\mathrm{d})$, and $\mathrm{R}(\mathrm{e})$.

2. $\exists x\left((e)_{0}=0 \& R_{1}\left(c,(e)_{1}, x\right) \&[x] \circledast C\right) \vee \exists x\left((e)_{0}=1 \& R_{1}\left(d,(e)_{1}, x\right)\right.$ $\&[\mathrm{x}] \circledast \mathrm{B}) \supset \exists \mathrm{y}\left(\exists \mathrm{x}\left(\left(\left(\left((\mathrm{e})_{0}=0 \& \mathrm{R}_{1}\left(\mathrm{c},(\mathrm{e})_{1}, \mathrm{x}\right)\right) \vee\left((\mathrm{e})_{0}=1 \& \mathrm{R}_{1}\left(\mathrm{~d},(\mathrm{e})_{1}, \mathrm{x}\right)\right)\right)\right.\right.\right.$ $\&[\mathrm{x}]=\mathrm{y}) \& \mathrm{y} \otimes C)$. Predicate calculus.

3. $\mathrm{H} \supset \mathrm{Gy}_{(\mathrm{Gx}}\left(\left((\mathrm{e})_{0}=0 \& \mathrm{R}_{1}\left(\mathrm{c},(\mathrm{e})_{1}, \mathrm{x}\right)\right) \vee\left((\mathrm{e})_{0}=1 \& \mathrm{R}_{1}\left(\mathrm{~d},(\mathrm{e})_{1}, \mathrm{x}\right)\right)\right)$ $\&[\mathrm{x}]=\mathrm{y}) \& \mathrm{y}(\mathrm{C}) .1$, unabbreviating the parts $\mathrm{o}(-), \mathrm{d}(-) ; 2$.

4. $\mathrm{H} \supset_{n}(n(\mathrm{c}, \mathrm{d}, \mathrm{e}) \circledast \mathrm{C})$. 3; Lemma 27; Lemma 15, corollary, 5.

5.* $\forall v\left(P(v) \supset \forall w\left(Q(w) \supset \forall z\left(R(z) \supset_{n}(n(v, w, z) @ C)\right)\right)\right)$. 4, definition of $\mathrm{H}$.

7.

Let the following abbreviation be made: $P(b)$ for $\forall x\left(x @ A \supset_{b}(b(x) \circledast B)\right)$, $Q(c)$ for $\forall y\left(y \circledast A \supset_{0}\left(\forall z\left(z \circledast B \supset_{\circ(y)}(c(y)(z) \circledast 1=0)\right)\right)\right)$, and $H$ for $P(b) \& Q(c)$ $\& \mathrm{a} \circledast \mathrm{A}$.

1. $H \supset_{b}(b(a) \circledast B) \& 。\left(\forall z\left(z @ B \supset_{c(a)}(c(a)(z) \circledast 1=0)\right)\right)$. Definition of $H$, $Q(c)$, and $P(b)$.

2. $\mathrm{H} \supset \forall z_{0}\left(z @ B \supset \supset_{0(a)}(c(a)(z) @ 1=0)\right) .1 ;$ Lemma $28,7$.

3. $\mathrm{H} \supset_{b}(\mathrm{o}(\mathrm{c}(\mathrm{a})(\mathrm{b}(\mathrm{a}))(1=0)) .1,2$; Lemma $28,1 \mathrm{~b}, 5 \mathrm{a}$.

4. $H \supset_{n}(n(b, c, a) \circledast 1=0) .3$; corollary to Lemma 27.

5.* $\forall t\left(P(t) \supset \forall v\left(Q(v) \supset \forall w\left(w @ A \supset_{n}(n(t, v, w) @ 1=0)\right)\right)\right) .4$, and definition of $\mathrm{H}$.

8.

Let $P(c)$ abbreviate $\forall x(x @ A \supset 。(c(x) \circledast 1=0))$.

1. $\mathrm{P}(\mathrm{c}) \& \mathrm{a} @ \mathrm{~A} \supset \mathrm{\supset}(\mathrm{c}(\mathrm{a}) \circledast 1=0)$. Definition of $\mathrm{P}(\mathrm{c})$.

2. $\mathrm{P}(\mathrm{c}) \& \mathrm{a} @ \mathrm{~A} \supset 1=0.1, \mathrm{D} 1$; Lemma $28,8,3 \mathrm{~b}$.

3. $\mathrm{P}(\mathrm{c}) \& \mathrm{a} @ \mathrm{~A} \supset_{n}(n(\mathrm{c}, \mathrm{a}) \circledast \mathrm{B}) .2$, Axiom 15, Predicate calculus.

4.* $\forall y\left(P(y) \supset \forall z\left(z @ A \supset_{n}(n(y, z) \circledast B)\right)\right) .3$.

10.

Let the free variables of $t$ be $e$, possibly an empty sequence. 
1. $\forall x_{c}(c(x) \circledast A(x)) \supset_{c}(c(t) \circledast A(t))$. Axiom 10.

2. $\quad \forall x_{0}(\mathrm{c}(\mathrm{x}) \circledast \mathrm{A}(\mathrm{x})) \supset_{\eta(n, e)}(\eta(n, e)(\mathrm{c}) @ \mathrm{~A}(\mathrm{t})) .1$; Corollary Lemma 27.

3. $\forall y\left(\forall x_{y}(y(x) \circledast A(x)) \supset_{\eta(n, e)}(\eta(n, e)(y) \circledast A(t))\right) .2$.

11.

Let the free variables of $t$ be $e$, again possibly an empty sequence.

1. $a ® A(t) \supset(\{t, a\})_{1} @ A\left((\{t, a\})_{0}\right)$. Lemma 10, 10.7.

2. $a \circledast A(t) \supset\{t, a\} \circledast \exists x A(x) .1, D 6$.

3. $\mathrm{a} \circledast \mathrm{A}(\mathrm{t}) \supset_{\eta(n, e)}(\eta(n, e)(\mathrm{a}) \circledast \exists \mathrm{x} A(\mathrm{x})) .2$; Lemma 27.

4. $\quad \forall y\left(y \circledast A(t) \supset_{\eta(n, e)}(\eta(n, e)(y) \circledast \exists x A(x)) .3\right.$.

13.

Let the following abbreviations be made: $F(a, b, c)$ for $(c)_{0}=\left\{0,\left\{(b)_{0}\right\}\right\}$ \& $\forall z\left(z \leqq c \quad \& a<z \supset(c)_{z}=0\right) \quad \& \forall z\left(z \leqq a \& 0<z \supset R_{1}\left((b)_{1}, \delta(z),(c)_{z 0}\right) \&\right.$ $\left.R_{1}\left(\left[(c)_{z 0}\right],\left[(c)_{\delta(z) 1}\right],(c)_{z 1}\right)\right), Q(c, p, d)$ for $F(a, b, c) \&\left[(c)_{p 1}\right]=d, H$ for $\forall u_{1}$ $\forall u_{2} \forall v_{1} \forall v_{2}\left(p \leqq a \& Q\left(u_{1}, p, v_{1}\right) \& Q\left(u_{2}, p, v_{2}\right) \supset v_{1}=v_{2}\right), t$ for $c \cdot \pi\left(a^{\prime}\right)^{\{d, f\}}, L$ for $F(a, b, c) \& R_{1}\left((b)_{1}, a, d\right) \& R_{1}\left([d],\left[(c)_{1}\right], f\right)$, and $P(b)$ for $(b)_{0} \circledast A(0)$ $\& \forall x_{(b)_{1}}\left(\forall y\left(y @ A(x) \supset_{(b)_{1}(x)}\left((b)_{1}(x)(y) @ A\left(x^{\prime}\right)\right)\right)\right)$.

1. $Q(c, 0, d) \supset d=(b)_{0}$. Definition of $Q(c, 0, d)$ and $F(a, b, c)$; Lemma 10, 10.7; Lemma 15, 10.

2. $Q\left(c_{1}, 0, d_{1}\right) \& Q\left(c_{2}, 0, d_{2}\right) \supset d_{1}=d_{2}$. 1, Schema 23 .

3. $Q\left(c, p^{\prime}, d\right) \supset F(a, b, c)$. Definition of $Q\left(c, p^{\prime}, d\right)$.

4. $F(a, b, c) \supset Q\left(c, p,\left[(c)_{p 1}\right]\right)$. Axiom 16, Definition $Q(c, p, d)$.

5. $\quad H \& p^{\prime} \leqq a \& Q\left(c_{1}, p^{\prime}, d_{1}\right) \& Q\left(c_{2}, p^{\prime}, d_{2}\right) \supset\left[\left(c_{1}\right)_{p 1}\right]=\left[\left(c_{2}\right)_{p 1}\right] .3,4$, definition of $\mathrm{H}$; Lemma $10,9.8$.

6. $\mathrm{p}^{\prime} \leqq \mathrm{a} \& \mathrm{Q}\left(\mathrm{c}, \mathrm{p}^{\prime}, \mathrm{d}\right) \supset \mathrm{R}_{1}\left((\mathrm{~b})_{1}, \mathrm{p},(\mathrm{c})_{\mathrm{p}^{\prime} 0}\right) \& \mathrm{R}_{1}\left(\left[(\mathrm{c})_{\mathrm{p}^{\prime} 0}\right],\left[(\mathrm{c})_{\mathrm{p}^{1}}\right],(\mathrm{c})_{\mathrm{p}^{\prime} \mathbf{1}}\right)$. Definition $Q\left(\mathrm{c}, \mathrm{p}^{\prime}, \mathrm{d}\right)$ and $\mathrm{F}(\mathrm{a}, \mathrm{b}, \mathrm{c})$; Lemma 10, 4.2, 9.9.

7. $\mathrm{H} \& \mathrm{p}^{\prime} \leqq \mathrm{a} \& \mathrm{Q}\left(\mathrm{c}_{1}, \mathrm{p}^{\prime}, \mathrm{d}_{1}\right) \& Q\left(\mathrm{c}_{2}, \mathrm{p}^{\prime}, \mathrm{d}_{2}\right) \supset\left[\left(\mathrm{c}_{1}\right)_{\mathrm{p}^{\prime} 0}\right]=\left[\left(\mathrm{c}_{2}\right)_{\mathrm{p}^{\prime} 0}\right]$ $\&\left[\left(c_{1}\right)_{p^{\prime} 1}\right]=\left[\left(c_{2}\right)_{p^{\prime} 1}\right] .5,6$; Corollary to Lemma 15, 5; Schema 23.

8. $H \& p^{\prime} \leqq a \& Q\left(c_{1}, p^{\prime}, d_{1}\right) \& Q\left(c_{2}, p^{\prime}, d_{2}\right) \supset d_{1}=d_{2}$. 7, definition $Q\left(c, p^{\prime}, d\right)$, Schema 23.

9. $\mathrm{p} \leqq \mathrm{a} \& Q\left(\mathrm{c}_{1}, \mathrm{p}, \mathrm{d}_{1}\right) \& Q\left(\mathrm{c}_{2}, \mathrm{p}, \mathrm{d}_{2}\right) \supset \mathrm{d}_{1}=\mathrm{d}_{2}$. 2, 8, Schema 13.

10. Эy $Q\left(y, a, d_{1}\right) \&$ By $Q\left(y, a, d_{2}\right) \supset d_{1}=d_{2}$. 9, Axiom 16, Lemma 10, 9.1. There exists a numeral $n$ such that for any formula $G(d)$ :

11. $\exists x\left(\exists y\left(F(a, b, y) \&\left[(y)_{a 1}\right]=x\right) \& G(x)\right) \equiv_{\{n, a\}}(G(\{n, a\}(b))) .10$, definition $Q(c, a, d)$; Lemma 27.

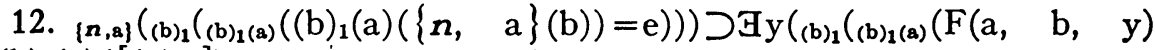
$\left.\left.\left.\&(b)_{1}(a)\left(\left[(y)_{a 1}\right]\right)=e\right)\right)\right) .11$; Schema 23, Lemma 28, 3b.

13. $\left.\{n, a\}(b)_{1}\left((b)_{1}(a)\left((b)_{1}(a)(\{n, a\}(b))=e\right)\right)\right) \supset \operatorname{GyHvHw}(F(a, \quad b, \quad y)$ $\left.\& R_{1}\left((b)_{1}, a, v\right) \& R_{1}\left([v],\left[(y)_{a 1}\right], w\right) \&[w]=e\right) .12$, unabbreviating the parts $\left(b_{1}(a)(-)\right.$ and $(b)_{1}(-)$ in the right member.

14. $c<g \supset(c)_{g}=0$. Lemma $10,10.10$.

15. $\mathrm{F}(\mathrm{a}, \mathrm{b}, \mathrm{c}) \& \mathrm{~g} \leqq \mathrm{c} \& \mathrm{a}<\mathrm{g} \supset(\mathrm{c})_{\mathrm{g}}=0$. Definition $\mathrm{F}(\mathrm{a}, \mathrm{b}, \mathrm{c})$.

16. $\mathrm{F}(\mathrm{a}, \mathrm{b}, \mathrm{c}) \& \mathrm{a}<\mathrm{g} \supset(\mathrm{c})_{\mathrm{g}}=0.14,15 ;$ Lemma $10,8.6,9.1$. 
17. $F(a, b, c) \supset(c)_{a^{\prime}}=0.16$; Lemma 10, 8.3.

18. $F(a, b, c) \supset 7 c=0$. Definition $F(a, b, c)$; Lemma 10, 10.9, 10.1 (contraposition).

19. $F(a, b, c) \supset 7 t=0.18$; definition $t$; Lemma $10,10.9,1.7$.

20. $F(a, b, c) \supset(t)_{a^{\prime}}=\{d, f\} .19,17$; Lemma 10, 10.5, 10.3.

21. $F(a, b, c) \supset \forall z\left(z \leqq a \supset(t)_{z}=(c)_{z}\right)$. 19; Lemma 10, 10.5, 9.5, 10.4.

22. $\mathrm{a}^{\prime}<\mathrm{g} \supset \mathrm{a}<\mathrm{g}$. Lemma $10,8.3,8.5$.

23. $F(a, b, c) \supset \forall z\left(a^{\prime}<z \supset(t)_{z}=0\right) .22,19,16$, definition $t$; Lemma 10, $10.5,8.8,10.4$.

24. $\mathrm{L} \supset \forall z\left(z \leqq t \& a^{\prime}<z \supset(t)_{z}=0\right) \& R_{1}\left((b)_{1}, \delta\left(a^{\prime}\right), \quad(t)_{a^{\prime} 0}\right) \& R_{1}\left(\left[(t)_{a^{\prime} 0}\right]\right.$, $\left.\left[(t)_{\delta\left(a^{\prime}\right) 1}\right],(t)_{a^{\prime} 1}\right) .23,21,20$, definition $L, t, F(a, b, c)$, Schema 23; Lemma 10, 4.2 .

25. $\mathrm{L} \supset \forall z\left(\mathrm{z} \leqq \mathrm{a} \& 0<\mathrm{z} \supset \mathrm{R}_{1}\left((\mathrm{~b})_{1}, \delta(\mathrm{z}),(\mathrm{t})_{\mathrm{z} 0}\right) \& \mathrm{R}_{1}\left(\left[(\mathrm{t})_{\mathrm{z} 0}\right],\left[(\mathrm{t})_{\delta(z) 1}\right],(\mathrm{t})_{z 1}\right)\right)$. 21, definition $\mathrm{L}, \mathrm{F}(\mathrm{a}, \mathrm{b}, \mathrm{c})$, Schema 23.

26. $\mathrm{L} \supset \mathrm{F}\left(\mathrm{a}^{\prime}, \mathrm{b}, \mathrm{t}\right) .24,25$, definition $\mathrm{L}, \mathrm{F}(\mathrm{a}, \mathrm{b}, \mathrm{c})$; Schema 23; Lemma 10, 9.4 .

27. $\mathrm{L} \&[\mathrm{f}]=\mathrm{e} \supset \mathrm{F}\left(\mathrm{a}^{\prime}, \mathrm{b}, \mathrm{t}\right) \&\left[(\mathrm{t})_{\mathbf{a}^{\prime}}\right]=$ e. 26, 20; Lemma 10, 10.7; Schema 23.

28. $\left.\{n, a\}(b)_{1}\left((b)_{1}(a)\left((b)_{1}(a)(\{n, a\}(b))=e\right)\right)\right) \cdot \supset_{\left\{n, a^{\prime}\right\}}\left(\left\{n, a^{\prime}\right\}(b)=e\right) .13,27$, 11.

29. $P(b) \supset(b)_{0} @ A(0)$. Definition $P(b)$.

30. $\{n, 0\}\left(\{n, 0\}(b)=(b)_{0}\right)$. 11, definition $F(0, b, c)$, substituting $\left\{\left\{0,\left\{(\mathrm{~b})_{0}\right\}\right\}\right\}$ for c; Lemma 10, 10.3, 10.4.

31. $P(b) \supset_{\{n, 0\}}(\{n, 0\}(b) @ A(0)) .29,30 ;$ Lemma 28, 1a.

32. $\mathrm{P}(\mathrm{b}) \&_{\{n, a\}}(\{n, a\}(b) ® A(a)) \supset\{n, a\}(b)_{1}(b)_{1}(a)\left((b)_{1}(a)(\{n, a\}(b))\right.$ (BA $\left.\left.\left(a^{\prime}\right)\right)\right)$ ). Definition $\mathrm{P}(\mathrm{b})$; Lemma 28, 7, 5b, 9a.

33. $P(b) \&_{\{n, a\}}(\{n, a\}(b) \circledast A(a)) \supset_{\left\{n, a^{\prime}\right\}}\left(\left\{n, a^{\prime}\right\}(b) \circledast A\left(a^{\prime}\right)\right)$. 28, 32, Corollary Lemma 28.

34. $P(b) \supset_{\{n, a\}}(\{n, a\}(b) \circledast A(a)) .31,33$, Schema 13.

35. $\forall z\left(z \circledast\left(A(0) \& \forall x\left(A(x) \supset A\left(x^{\prime}\right)\right)\right) \supset_{\{n, a\}}(\{n, a\}(z) \circledast A(a))\right)$. 34, definition $\mathrm{P}(\mathrm{b}), \mathrm{D} 2, \mathrm{D} 7, \mathrm{D} 4$.

14.

1. $c=0 \& a^{\prime}=b^{\prime} \supset \exists x(c=c \& x=0) \& a=b$. Axiom 14 .

2. $c=0 \& a^{\prime}=b^{\prime} \supset_{n}(n(c)=0 \& a=b)$. 1, Lemma 27 .

3. $\forall y\left(y=0 \& a^{\prime}=b^{\prime} \supset_{n}(n(y)=0 \& a=b)\right) .2$.

15.

1. $\mathrm{c}=0 \& \mathrm{a}^{\prime}=0 \supset_{n}(n(\mathrm{c})=0 \& 1=0)$. Axiom 15, Schema 8 .

2. $\forall y\left(y=0 \& a^{\prime}=0 \supset_{n}(n(y)=0 \& 1=0)\right.$. 1 .

17.

1. $d=0 \& a=b \supset(e=0 \& a=c \supset \forall x(d=d \& e=e \& x=0) \& b=c)$. Axiom 17. 
2. $\mathrm{d}=0$ \& $\mathrm{a}=\mathrm{b} \supset\left(\mathrm{e}=0 \& \mathrm{a}=\mathrm{c} \supset_{n}(n(\mathrm{~d}, \mathrm{e})=0 \& \mathrm{~b}=\mathrm{c})\right)$. 1, Lemma 27 . 3.* $\forall y\left(y=0 \& a=b \supset \forall z\left(z=0 \& a=c \supset_{n}(n(y, z)=0 \& b=c)\right)\right) .2$.

18.

Similar to 14.

23.

1. $a=0 \& b=c \supset(d \circledast A(b) \supset \exists x(a=a \& d=x \& x ® A(c)))$. Schema 23.

2. $a=0 \& b=c \supset\left(d @ A(b) \supset_{n}(n(a, d) \circledast A(c))\right)$. 1, Lemma 27.

3.* $\forall y\left(y=0 \& b=c \supset \forall z\left(z @ A(b) \supset_{n}(n(y, z) @ A(c))\right)\right) .2$.

Theorem 4. For every formula $\mathrm{A}(\mathfrak{a})$ containing exactly the free variables exhibited and provable in $S_{3}$, there exists a numeral $n$ such that ${ }_{n}(n(\mathfrak{a}) \circledast \mathrm{A}(\mathfrak{a}))$ is provable in $S_{3}$.

The result for the axioms of $S_{3}$ follows immediately from the preceding lemma, where $n$ is chosen according to Lemma 27 so that ${ }_{n}\left(n(\mathfrak{a})=(\{t, \mathfrak{a}\})_{0}\right)$ is provable in $S_{3}$.

To complete the demonstration by induction we must consider the rules of inference of $S_{1}: 2,9$, and 12 .

For Rule 2, suppose that $b$ is a list of the free variables of $A \supset B, \mathfrak{a}$ of $A$, and $\mathfrak{b}$ of $B$; and that ${ }_{n}(n(\mathfrak{a}) \circledast A)$ and $m\left(\forall x\left(x @ A \supset_{m(\mathfrak{b})}(m(\mathfrak{b})(x) \circledast B)\right)\right)$ are provable in $S_{3}$. Then $\forall x\left(x(\circledR A) \supset_{m}(m(b)(m(b)(x) \circledast B))\right)$ is provable in $S_{3}$ (Lemma $28,7,5 \mathrm{~b})$. Hence the formula ${ }_{n}\left({ }_{m}(m(\mathfrak{b})(m(\mathfrak{b})(n(\mathfrak{a})) @ B))\right.$ is provable in $S_{3}$ (Lemma 28, 1b, 5a). Replacing the free variables in $\mathfrak{b}$ and $\mathfrak{a}$ which do not appear in $\mathfrak{b}$ by numerals and applying the corollary to Lemma 27 , there exists a numeral $p$ such that ${ }_{p}(p(\mathfrak{b}) \circledast B)$ is provable in $S_{3}$.

For Rule 9, suppose the free variables of the formula $C \supset A(b)$ are $a, b$, that $C$ does not contain $b$, and that the formula $n\left(\forall y\left(y \circledast C \supset_{n(a, b)}(n(a, b)(y)\right.\right.$

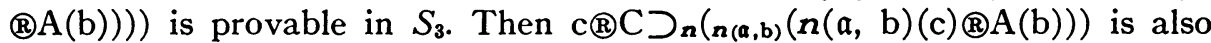
provable in $S_{3}$ (Lemma $28,7,5 \mathrm{~b}$ ). Applying the corollary to Lemma 27 , there exists a numeral $p$ such that $\mathrm{c} \circledast \mathrm{C} \supset_{\{p, a\}}(\{p, a\}(\mathrm{c}, \mathrm{b}) \circledast \mathrm{A}(\mathrm{b}))$ is provable in $S_{\mathbf{3}}$, and since $C$ does not contain $b, \forall y\left(y @ C \supset \forall x_{\{p, a\}}(\{p, a\}(y, x) \circledast A(x))\right)$, and finally by application of Lemma $30,\left\{s_{1},\{p, a\}\right\} ®(C \supset \forall x A(x))$. The case may be completed by application of Lemma 27 .

For Rule 12, suppose the free variables of $A(b) \supset C$ are $a, b$, where again $C$ does not contain $b$, and that the formula $n\left(\forall y\left(y @ A(b) \supset_{n(a, b)}(n(a, b)(y) \circledast C)\right)\right)$

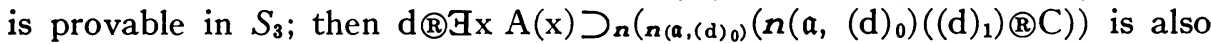
provable in $S_{3}$, Lemma $28,7,5 \mathrm{~b}$; and applying the corollary to Lemma 27 , since $C$ does not contain $b$, we have $d \AA \exists x A(x) \supset\{p, a\}(\{p, a\}(d) \circledast C)$. The case is completed in the same manner as the preceding one.

Corollary 4.1. If $\mathrm{A}$ is a formula deducible in $S_{3}$ from formulas $\mathrm{A}_{1}, \cdots, \mathrm{A}_{k}$, $k \geqq 0$, and if $\mathrm{B}, \mathrm{B}_{1}, \cdots, \mathrm{B}_{k}$ are the respective closures of $\mathrm{A}, \mathrm{A}_{1}, \cdots, \mathrm{A}_{k}$ with respect to the free variables of $\mathrm{A}_{1}, \cdots, \mathrm{A}_{k}$ not held constant in the deduction, then $\mathrm{\exists} \mathrm{y}(\mathrm{y} \circledast \mathrm{B})$ is deducible in $S_{3}$ from $\mathrm{B}_{\mathrm{1}}\left(\mathrm{y}_{1}{ }^{\circledR} \mathrm{B}_{1}\right), \cdots, \exists \mathrm{y}_{k}\left(\mathrm{y}_{k}{ }^{\circledR} \mathrm{B}_{k}\right)$. 
By the deduction theorem, the formula $\mathrm{B}_{1} \& \cdots \& \mathrm{~B}_{k} \supset \mathrm{B}$ is provable in $S_{3}$. If $\mathrm{b}$ is the list of free variables in this formula, then there exists a numeral $n$ such that $n\left(b \circledast\left(\mathrm{B}_{1} \&\left(\cdots \& \mathrm{~B}_{k} \cdots\right)\right) \supset_{\boldsymbol{n}(\mathfrak{b})}(\boldsymbol{n}(\mathfrak{b})(\mathrm{b}) \circledast \mathrm{B})\right)$ is provable in $S_{3}$. Hence, by Lemma $28,5 \mathrm{~b}$, the formula $\mathrm{b} \circledast\left(\mathrm{B}_{1} \&\left(\cdots \& \mathrm{~B}_{k} \cdots\right)\right)$ $\supset_{n}(n(b)(n(b)(b) ® B))$ is also provable in $S_{3}$, and finally, b® $\left(B_{1} \&(\cdots\right.$ $\left.\left.\& \mathrm{~B}_{k} \cdots\right)\right) \supset \mathrm{Hy}(\mathrm{y} \circledast \mathrm{B})($ Lemma 28, 8). Then applying $\mathrm{D} 2$, we have the for-

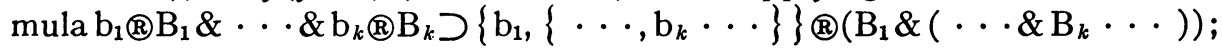
and hence

$$
\mathrm{H}_{1}\left(\mathrm{y}_{1} \circledast \mathrm{B}_{1}\right) \& \cdots \& \mathrm{H}_{\mathrm{k}}\left(\mathrm{y}_{k} \circledast \mathrm{B}_{k}\right) \supset \mathrm{Hy}(\mathrm{y} \circledast \mathrm{B}) .
$$

Since A does not (except in the elementary case) constitute a consecutive part of a $₫ A$, the replacement rule requires special justification as applied to $\mathrm{a} \circledast \mathrm{A}$. This we are now able to give.

COROLlary 4.2. For any formulas $\mathrm{A}$ and $\mathrm{B}$ of $S_{3}, \mathrm{By}(\mathrm{y} \circledast \mathrm{A}) \equiv \mathrm{By}(\mathrm{y} \circledast \mathrm{B})$ is deducible from $\mathrm{A} \equiv \mathrm{B}$ in $S_{3}$.

Proof is immediate from Corollary 4.1 and the deduction theorem for $S_{30}$

THEOREM 5. For every formula $\mathrm{A}$ of $S_{3}$, there exists a numeral $\mathrm{n}$ such that the following formulas are provable in $S_{3}$ :

$$
\begin{aligned}
a \circledast A & \supset n(n(a) \circledast(a @ A)), \\
c((a ß A) & \supset a(A .
\end{aligned}
$$

The proof is by induction on the number of logical symbols in A. For proper choice of the numerals in each of the cases, the following formulas are provable.

Basis. Assume that $A$ is an elementary formula.

1.1. $a=0 \& A \supset\{0, a\}(a=0 \& A)$. D1, D2; Lemma 10, 10.7.

1.2. $\mathrm{a}=0 \& \mathrm{~A} \supset_{n}(n(a) \circledast(a=0 \& A)) .1 .1$; Lemma 27 .

2.1. $\mathrm{c}\left(\mathrm{a}(\mathrm{a}=0 \& \mathrm{~A}) \supset(\mathrm{c})_{0}=0 \& \mathrm{a}=0 \&(\mathrm{c})_{1}=0 \& \mathrm{~A}\right.$. D2, D1.

2.2. $c @(a=0 \& A) \supset a=0 \& A .2 .1$.

For the induction step, suppose there exist a numeral $\boldsymbol{n}$ and a numeral $\boldsymbol{m}$ such that, in addition to (i) and (ii), the following formulas are provable in $S_{3}$ :

$$
\begin{aligned}
b \circledast B & \supset \boldsymbol{m}(\boldsymbol{m}(\mathrm{b}) \circledast(\mathrm{b} \circledast \mathrm{B})), \\
\mathrm{d}(\mathrm{b}(\mathrm{b} B) & \supset \mathrm{b} \circledast \mathrm{B} .
\end{aligned}
$$

Then we consider cases for each of the logical symbols.

Conjunction.

1.1. $\quad c ®(A \& B) \supset_{n}\left(n\left((c)_{0}\right) \circledast\left((c)_{0} \circledast A\right)\right) \&_{m}\left(m\left((c)_{1}\right) \circledast\left((c)_{1} \circledast B\right)\right)$. (i), (iii), D2.

1.2. $\quad \mathrm{c} \circledast(\mathrm{A} \& \mathrm{~B}) \supset_{n}\left(m_{m}\left(\left\{n\left((\mathrm{c})_{0}\right), m\left((\mathrm{c})_{1}\right)\right\} \circledast(\mathrm{c} @(\mathrm{~A} \& \mathrm{~B}))\right)\right) . \quad \mathrm{D} 2, \quad 1.1$; Lemma 28, 3b; Lemma 10, 10.7; Schema 23.

1.3. $\mathrm{c} @(\mathrm{~A} \& \mathrm{~B}) \supset_{p}(p(\mathrm{c}) \AA(\mathrm{c}(\mathrm{A} \& \mathrm{~B}))) .1 .2$; corollary to Lemma 27. 
2.1. $d \circledast(c ®(A \& B)) \supset(d)_{0} \circledast\left((c)_{0} @ A\right) \&(d)_{1} \circledast\left((c)_{1} \circledast B\right) . D 2$.

2.2. $\mathrm{d} \circledast(\mathrm{c} @(A \& B)) \supset c ®(A \& B) .2 .1$, (ii), (iv), $D 2$.

Disjunction. Let the following abbreviations be made:

$F(c, d)$ for $_{n}\left((c)_{0}=0 \& n\left((c)_{1}=d\right) \bigvee_{m}\left(7 .(c)_{0}=0 \& m\left((c)_{1}\right)=d\right), H\right.$ for $(c)_{0}=0$ $\&(\mathrm{c})_{1} \circledast \mathrm{A}$, and $\mathrm{t}$ for $\left\{(\mathrm{c})_{0},\{0, k(\mathrm{c})\}\right\}$, where $k$ is introduced below.

1.1. $F\left(c, d_{1}\right) \& F\left(c, d_{2}\right) \supset d_{1}=d_{2}$. Lemma $10,1.0 ;$ corollary to Lemma 15, 5 , functional abbreviation.

There exists a numeral $k$ such that for any formula $G(d)$ the following is provable in $S_{3}$ :

1.2. $\quad \exists x\left(F(c, x) \& G(x) \equiv_{k}(G(k(c))) .1 .1\right.$; Lemma 27 .

1.3. $\mathrm{H} \supset(\mathrm{c})_{0}=0 \&_{n}\left(n\left((\mathrm{c})_{1}\right) \circledast\left((\mathrm{c})_{1} \circledast \mathrm{A}\right)\right)$. Formula (i), definition of $\mathrm{H}$. 8.

1.4. $\mathrm{H} \supset \mathrm{Gx}\left({ }_{n}\left((\mathrm{c})_{0}=0 \& n\left((\mathrm{c})_{1}\right)=\mathrm{x}\right) \& \mathrm{x}\left(\left((\mathrm{c})_{1} \circledast \mathrm{A}\right)\right)\right.$. 1.3; Lemma 28, 3b,

1.5. $H \supset \exists x\left(F(c, x) \& x \circledast\left((c)_{1} \circledast A\right)\right)$. 1.4, definition $F(c, d)$.

1.6. $\mathrm{H} \supset_{k}\left((\mathrm{c})_{0}=0 \& k(\mathrm{c}) \circledast\left((\mathrm{c})_{1} \circledast \mathrm{A}\right)\right)$. 1.5, 1.2, definition of $\mathrm{H}$; Lemma $28,3 \mathrm{~b}$.

1.7. $(\mathrm{c})_{0}=1 \&(\mathrm{c})_{1} \circledast \mathrm{B} \supset_{k}\left((\mathrm{c})_{0}=1 \& k(\mathrm{c}) \circledast\left((\mathrm{c})_{1} @ \mathrm{~B}\right)\right)$. Similar to the proof of 1.6 .

1.8. $\mathrm{c} \circledast(\mathrm{A} \vee \mathrm{B}) \supset_{k}\left(\left((\mathrm{c})_{0}=0 \& 0=0 \&(\mathrm{c})_{0}=0 \& k(\mathrm{c}) \circledast\left((\mathrm{c})_{1} \circledast \mathrm{A}\right)\right) \backslash\left((\mathrm{c})_{0}\right.\right.$ $\left.\left.=1 \& 1=1 \&(\mathrm{c})_{0}=1 \& k(\mathrm{c}) \circledast\left((\mathrm{c})_{1} \circledast \mathrm{B}\right)\right)\right)$. 1.6, 1.7, D3, Axiom 16; Lemma $28,3 \mathrm{~b}, 4 \mathrm{a}$.

1.9. $c ®(A \vee B) \supset_{k}\left(\left((t)_{0}=0 \&(t)_{10}=0 \&(\mathrm{c})_{0}=0 \&(\mathrm{t})_{11} \AA\left((\mathrm{c})_{1} \AA \mathrm{A}\right)\right)\right.$ $V\left((t)_{0}=1 \&(t)_{10}=0 \&(c)_{0}=1 \&(t)_{11}\left(\left((c)_{1} \circledast B\right)\right)\right)$. 1.8, definition of $t$; Lemma 10, 10.7; corollary to Lemma 28.

1.10. $c ®(A \vee B) \supset_{k}(t \circledast(c @(A \vee B)))$. D2, D3.

1.11. $c ®(A \vee B) \supset_{p}(p(c) \circledast(c ®(A \vee B)))$. Corollary to Lemma 27:

2.1. $\mathrm{d} \circledast(\mathrm{c} \circledast(\mathrm{A} \backslash \mathrm{B})) \supset\left(\left((\mathrm{d})_{0}=0 \&(\mathrm{~d})_{1} \AA\left((\mathrm{c})_{0}=0 \&(\mathrm{c})_{1} @ \mathrm{~A}\right)\right) \vee\left((\mathrm{d})_{0}\right.\right.$ $\left.\left.=1 \&(\mathrm{~d})_{1} @\left((\mathrm{c})_{0}=1 \&(\mathrm{c})_{1} @ \mathrm{~B}\right)\right)\right)$. D3.

2.2. $\mathrm{d} @(\mathrm{c} @(\mathrm{~A} \vee \mathrm{B})) \supset\left((\mathrm{c})_{0}=0 \&(\mathrm{c})_{1} @ \mathrm{~A}\right) \vee\left((\mathrm{c})_{0}=1 \&(\mathrm{c})_{1} \circledast \mathrm{B}\right) .2 .1, \mathrm{D} 3$, D1, (ii) and (iv).

2.3. $\mathrm{d} \circledast(\mathrm{c} \circledast(\mathrm{A} \vee \mathrm{B})) \supset \mathrm{c} \circledast(\mathrm{A} \vee \mathrm{B}) .2 .2, \mathrm{D} 3$.

Implication. Let $F$ abbreviate $\exists y\left(R_{1}(c, a, y) \&[y] \circledast B\right)$ and let $t$ abbreviate $\{\boldsymbol{r}(\mathrm{c}, \mathrm{a}),\{0, \boldsymbol{m}([\boldsymbol{r}(\mathrm{c}, \mathrm{a})])\}\}$, where $\boldsymbol{r}$ is defined below.

1.1. $c ®(A \supset B) \supset \forall x\left(x @ A \supset \exists y\left(R_{1}(c, x, y) \&[y] \circledast B\right)\right) . D 4$.

1.2. $\mathrm{d} \circledast(\mathrm{a} \circledast \mathrm{A}) \supset \mathrm{a} \circledast \mathrm{A}$. (ii).

1.3. $c ®(A \supset B) \& d @(a @ A) \supset F .1 .1,1.2$.

There exists a numeral $r$ such that, for every formula $G(d)$, the following is provable:

1.4. $\operatorname{By}\left(\mathrm{R}_{\mathbf{1}}(\mathrm{c}, \mathrm{a}, \mathrm{y}) \& \mathrm{G}(\mathrm{y})\right) \equiv_{\boldsymbol{r}}(\mathrm{G}(\boldsymbol{r}(\mathrm{c}, \mathrm{a})))$. Corollary to Lemma 15, 5; Lemma 27.

1.5. $\mathrm{F} \supset_{r}\left([r(\mathrm{c}, \mathrm{a})]_{\circledast} \mathrm{B}\right)$. 1.4, definition of $\mathrm{F}$.

1.6. $\left.F \supset_{r}(m(m)([r(c, a)]) \circledast([r(c, a)] \circledast B))\right) \cdot 1.5$, (iii); Lemma 28, 1b, 5a.

1.7. $F \supset_{r}\left(R_{1}(c, a, r(c, a))\right)$. Definition of $F, 1.4$. 
1.8. $\mathrm{F} \supset_{r}\left({ }_{m}\left(R_{1}(c, a, r(c, a)) \& m([r(c, a)]) @([r(c, a)] ® B)\right)\right) .1 .6,1.7$; Lemma $28,3 \mathrm{a}, 3 \mathrm{~b}$.

1.9. $F \supset_{r}\left({ }_{m}\left(0=0 \& R_{1}(c, a, r(c, a)) \& \boldsymbol{m}([r(c, a)]) ®([r(c, a)] ® B)\right)\right) .1 .8$, Axiom 16; Lemma 28, 3b.

1.10. $\mathrm{F} \supset_{\boldsymbol{x}}\left({ }_{m}\left((\mathrm{t})_{10}=0 \& \mathrm{R}_{1}\left(\mathrm{c}, \mathrm{a},(\mathrm{t})_{0}\right) \&(\mathrm{t})_{11} \AA\left(\left[(\mathrm{t})_{0}\right]_{\circledast} \mathrm{B}\right)\right)\right)$. 1.9, definition $\mathrm{t}$; Lemma 10, 10.7; corollary to Lemma 28.

1.11. $F \supset_{r}\left(m\left(t \circledast \exists y\left(R_{1}(c, a, y) \&[y] \circledast B\right)\right)\right)$. D6, D1, D2.

1.12. $F \supset_{p}\left(p \cdot(c, a, d) \circledast \exists y\left(R_{1}(c, a, y) \&[y] \circledast B\right)\right)$. 1.11 ; corollary to Lemma 27.

1.13. $c ®(A \supset B) \supset \forall x \forall z\left(z \circledast(x ® A) \supset_{p}\left(p(c, x, z) \circledast \exists y\left(R_{1}(c, x, y) \&[y]\right.\right.\right.$ (B)))). 1.3, 1.12.

The desired formula of the form of Formula (i) is obtained from 1.13 by application of Lemma 30, D4, D7, and the functional abbreviation.

2.1. $\mathrm{d} \circledast(\mathrm{c} \circledast(\mathrm{A} \supset \mathrm{B})) \supset \forall \mathrm{x}_{\mathrm{d}}\left(\mathrm{\forall z}\left(\mathrm{z} \circledast(\mathrm{x} \circledast \mathrm{A}) \supset_{\mathrm{d}(\mathrm{x})}\left((\mathrm{d}(\mathrm{x})(\mathrm{z}))_{10}=0 \& \mathrm{R}_{1}\right.\right.\right.$ $\left.\left.\left(\mathrm{c}, \mathrm{x},(\mathrm{d}(\mathrm{x})(\mathrm{z}))_{0}\right) \&(\mathrm{~d}(\mathrm{x})(\mathrm{z}))_{11}\left(\left(\left[(\mathrm{~d}(\mathrm{x})(\mathrm{z}))_{0}\right] ® \mathrm{~B}\right)\right)\right)\right)$. D4, D7, D6, D2, D1, functional abbreviation.

2.2. $\mathrm{d} \circledast(\mathrm{c} \circledast(\mathrm{A} \supset \mathrm{B})) \& \mathrm{a} \circledast \mathrm{A} \supset \mathrm{n}_{\mathrm{n}}\left(\mathrm{d}\left(\mathrm{d}(\mathrm{a})\left(\mathrm{d}(\mathrm{a})(n(\mathrm{a}))_{10}=0 \& \mathrm{R}_{1}(\mathrm{c}, \mathrm{a}\right.\right.\right.$, $\left.\left.\left.\left.(\mathrm{d}(\mathrm{a})(n(\mathrm{a})))_{0}\right) \&(\mathrm{~d}(\mathrm{a})(\boldsymbol{n}(\mathrm{a})))_{11} @\left(\left[(\mathrm{~d}(\mathrm{a})(\boldsymbol{n}(\mathrm{a})))_{0}\right] ® \mathrm{~B}\right)\right)\right)\right)$. 2.1, (i); Lemma 28, $9 \mathrm{a}, 5 \mathrm{~b}, 5 \mathrm{a}$.

2.3. $\mathrm{d} \circledast(\mathrm{c} \circledast(\mathrm{A} \supset \mathrm{B})) \& \mathrm{a} \circledast \mathrm{A} \supset_{k}\left((k(\mathrm{~d}, \mathrm{a}))_{10}=0 \& \mathrm{R}_{1}\left(\mathrm{c}, \quad \mathrm{a}, \quad(k(\mathrm{~d}, \mathrm{a}))_{0}\right)\right.$ $\left.\&(k(d, a))_{11} \circledast\left(\left[(k(d, a))_{0}\right] \circledast B\right)\right) .2 .2$; corollary to Lemma 27.

2.4. $d \AA(c \AA(A \supset B)) \& a \circledast A \supset \exists w \quad \exists y\left(R_{1}(c, a, y) \& w \circledast([y] \circledast B)\right)$. 2.3; Lemma 28, 9b.

2.5. $\mathrm{d} \circledast(\mathrm{c} @(A \supset \mathrm{B})) \& \mathrm{a} \circledast \mathrm{A} \supset \mathrm{By}\left(\mathrm{R}_{1}(\mathrm{c}, \mathrm{a}, \mathrm{y}) \&[\mathrm{y}] \circledast \mathrm{B}\right) .2 .4$, (iv).

2.6. $\mathrm{d} @(\mathrm{c} @(\mathrm{~A} \supset \mathrm{B})) \supset \mathrm{Vx}(\mathrm{x} \circledast \mathrm{A} \supset \mathrm{o}(\mathrm{c}(\mathrm{x}) \circledast \mathrm{B}))$. 2.5.

Negation. The case for negation is an instance of that for implication.

Universal quantifier. Let $t$ abbreviate $\{\boldsymbol{r}(\mathrm{c}, \mathrm{a}),\{\boldsymbol{0}, \boldsymbol{n}([\boldsymbol{r}(\mathrm{c}, \mathrm{a})])\}\}$.

1.1. $\quad c @ \forall x A(x) \supset \forall x \exists y\left(R_{1}(c, x, y) \&[y] \circledast A(x)\right)$. D7.

1.2. $\mathrm{c} @ \forall x A(x) \supset_{r}\left(n\left(0=0 \& R_{1}(c, a, r(c, a)) \& n([r(c, a)]) \circledast([r(c, a)]\right.\right.$ $(\circledR A(a))))$. By steps similar to 1.4-1.9 of the implication case, using here 1.1 and (i).

1.3. $\quad c \circledast \forall x A(x) \supset_{r}\left(n\left((t)_{10}=0 \& R_{1}\left(c, a, \quad(t)_{0}\right) \&(t)_{11}\left(\left(\left[(t)_{0}\right] \circledast A(a)\right)\right)\right)\right.$. 1.2 ; Lemma 10, 10.7; corollary to Lemma 28.

1.4. $c @ \forall x A(x) \supset_{r}\left(n\left(t \circledast B y\left(R_{1}(c, a, y) \&[y] @ A(a)\right)\right)\right)$. D6, D2, D1.

1.5. $c \circledast \forall x A(x) \supset_{p}\left(p(c, a) \circledast H y\left(R_{1}(c, a, y) \&[y] \circledast A(a)\right)\right)$. 1.4; corollary to Lemma 27.

This portion of the case may be completed by application of Lemma 30 .

2.1. $\mathrm{d} \circledast(\mathrm{c} @ \forall \mathrm{xA}(\mathrm{x})) \supset \forall \mathrm{x}_{\mathrm{d}}\left((\mathrm{d}(\mathrm{x}))_{10}=0 \& \mathrm{R}_{1}\left(\mathrm{c}, \mathrm{x}, \quad(\mathrm{d}(\mathrm{x}))_{0}\right) \&(\mathrm{~d}(\mathrm{x}))_{11}\right.$ (B) $\left.\left(\left[(\mathrm{d}(\mathrm{x}))_{0}\right] ® \mathrm{~A}(\mathrm{x})\right)\right) . \mathrm{D} 7, \mathrm{D} 6, \mathrm{D} 2, \mathrm{D} 1$.

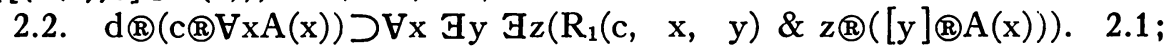
Lemma $28,9 \mathrm{~b}$.

2.3. $\mathrm{d} \circledast(\mathrm{c} @ \forall x A(x)) \supset \forall x \rightarrow y\left(R_{1}(c, x, y) \&[y] \circledast A(x)\right)$. 2.2, (ii).

Existential quantifier. 
1.1. $\quad(\mathrm{a})_{1} @ \mathrm{~A}\left((\mathrm{a})_{0}\right) \supset_{n}\left(n\left((\mathrm{a})_{1}\right) \circledast\left((\mathrm{a})_{1} @ \mathrm{~A}\left((\mathrm{a})_{0}\right)\right)\right.$. (i).

1.2. $\mathrm{a} \circledast \exists \mathrm{xA}(\mathrm{x}) \supset_{n}\left(n\left((\mathrm{a})_{1}\right) \circledast(\mathrm{a} \circledast \mathrm{BxA}(\mathrm{x}))\right.$. D6.

1.3. $\mathrm{a} \circledast \mathrm{B} \mathrm{xA}(\mathrm{x}) \supset_{p}(p(\mathrm{a}) \circledast(\mathrm{a} \circledast \mathrm{HxA}(\mathrm{x}))$. 1.2 ; corollary to Lemma 27.

2.1. $\mathrm{k} \circledast\left((\mathrm{a})_{1} \circledast \mathrm{A}\left((\mathrm{a})_{0}\right) \supset(\mathrm{a})_{1} \circledast \mathrm{A}\left((\mathrm{a})_{0}\right)\right.$. (ii).

2.2. $\mathrm{k} \circledast(\mathrm{a} \circledast \exists \mathrm{xA}(\mathrm{x})) \supset \mathrm{a} @ \exists \mathrm{xA}(\mathrm{x}) .2 .1, \mathrm{D} 6$. in $S_{3}$ :

CoRollary. For every formula A of $S_{3}$ the following formulas are provable

$$
\begin{aligned}
& \text { ' } \\
& \exists y(y \circledast(G x(x ® A) \supset A)) \text {. }
\end{aligned}
$$

Furthermore, if $\mathfrak{y}$ are the free variables of $\mathrm{A}$, the following formulas are also provable in $S_{3}$ :

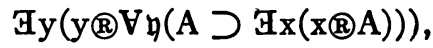

$$
\begin{aligned}
& \exists y(y @ V y(H x(x ® A) \supset A)) \text {. }
\end{aligned}
$$

For every $A$ there exist an $n, a k$, and a $p$ such that the following formulas are provable in $S_{3}$ :

1.1. $a @ A \supset_{n}(n(a) \circledast(a @ A))$. Theorem 5 .

1.2. $a ® A \supset_{n}\left((\{a, n(a)\})_{i}\left((\{a, n(a)\})_{0}(A)\right)\right.$. 1.1; Lemma 10, 10.7; corollary to Lemma 28 .

1.3. $\mathrm{a} \circledast \mathrm{A} \supset_{n}(\{\mathrm{a}, \boldsymbol{n}(\mathrm{a})\} \circledast(\exists \mathrm{x}(\mathrm{x} \circledast \mathrm{A})))$. 1.2, D6.

1.4. $\mathrm{a} @ A \supset_{p}(p(a) \circledast(\exists x(x ® A)))$. 1.3; corollary to Lemma 27.

1.5. $p \circledast(\mathrm{A} \supset \exists \mathrm{x}(\mathrm{x} \circledast \mathrm{A}))$. 1.4, $\mathrm{D} 4$.

2.1. $b \circledast(\exists x(x @ A)) \supset(b)_{1}\left((b)_{0}(A)\right.$. D6.

2.2. $\mathrm{b} \circledast(\exists \mathrm{x}(\mathrm{x} \circledast \mathrm{A})) \supset(\mathrm{b})_{0} \AA \mathrm{A}$. 2.1 ; Theorem 5 .

2.3. $\mathrm{b} \circledast(\exists \mathrm{x}(\mathrm{x} \circledast \mathrm{A})) \supset_{k}(k(\mathrm{~b}) \circledast \mathrm{A})$. 2.2; Lemma 27.

2.4. $k \circledast(\exists x(x \circledast A) \supset A) .2 .3, D 4$.

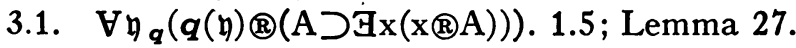

Formula (iii) follows from 3.1 by application of Lemma 30 . The proof of (iv) from 2.4 is similar.

THEOREM 6. If A is a formula of $S_{3}$, then the following formulas are provable in $S_{3}$ :

$$
\begin{aligned}
& \text { 7月 } x(x @ A) \supset \exists x(x ® 7 A),
\end{aligned}
$$

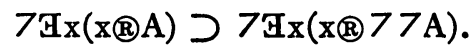

1. $7(a @ A) \supset\left(a @ A \supset_{n}(n(a) \circledast 1=0)\right)$. Schema 8.

2. $\forall x>(x @ A) \supset \forall x\left(x @ A \supset_{n}(n(x) @ 1=0)\right) .1$.

3. $\forall x>(x \circledast A) \supset n \circledast 7 A .2, D 5$.

4. $7 \mathrm{H} x(x @ A) \equiv \forall x>(x \circledast A)$. Predicate calculus.

5. $7 \exists x(x \circledast A) \supset \exists x(x \circledast 7 A) .3,4$.

6. $b ® 77 A \supset \forall x(x ® 7 A \supset b(b(x)=0 \& 1=0))$. D5, D1. 
7. $a \circledast 7 A \& b \circledast 77 A \supset b(b(a)=0 \& 1=0) .6$.

8. $a ® 7 A \& \exists x(x @ 77 A) \supset 1=0.7$; Lemma $28,3 b$.

9. $\exists x(x @ 7 A) \supset 7 \exists x(x ® 77 A) .8$, Axiom 15 , Schema 8.

10. $7 \exists x(x ® A) \supset 7 \exists x(x ® 7>A) .5,8$.

LeMma 32. The following formulas are provable in $S_{3}$ :

(i) $c @ \forall z>R_{1}(a, b, z) \supset \forall z>R_{1}(a, b, z)$.

(ii) $\exists y\left(R_{1}(a, b, y) \&\left(\forall z>R_{1}(a, b, z) \bigvee\left(([y])_{0}=1 \& R_{1}\left(a, b,([y])_{10}\right)\right)\right) \supset 1\right.$ $=0$.

1. $c @ \forall z>R_{1}(a, b, z) \supset \forall z z_{0}\left(\forall x\left(x=0 \& R_{1}(a, b, z) \supset_{o(z)}(c(z)(x)=0 \& 1\right.\right.$ $=0))$ ). D7, D5, D1.

2. $c @ \forall z>R_{1}(a, b, z) \supset_{0}\left(0=0 \& R_{1}(a, b, d) \supset_{o(d)}(c(d)(0)=0 \& 1=0)\right) .1$.

3. $c ® V z>R_{1}(a, b, z) \supset\left(R_{1}(a, b, d) \supset 1=0\right)$. 2 ; Lemma 28, 5b, 3b.

From 3 we easily obtain (i).

4. $R_{1}(a, b, d) \& \forall z>R_{1}(a, b, z) \supset 1=0$.

5. $R_{1}(a, b, d) \& R_{1}\left(a, b,([d])_{10}\right) \supset d=([d])_{10}$. Corollary to Lemma 15,5 .

6. $\mathrm{d}=([\mathrm{d}])_{10} \supset \mathrm{d}=0$. Lemma $10,10.6,10.1,8.5,8.8,1.0 ;$ Lemma $15,10$.

7. $([\mathrm{d}])_{0}=1 \supset 7 \mathrm{~d}=0$. Lemma $10,10.1 ;$ Lemma 15,10 .

8. $R_{1}(a, b, d) \&([d])_{0}=1 \& R_{1}\left(a, b,([d])_{10}\right) \supset 1=0.5,6,7$.

9. $\mathrm{R}_{1}(\mathrm{a}, \mathrm{b}, \mathrm{d}) \&\left(\mathrm{\forall z}>\mathrm{R}_{1}(\mathrm{a}, \mathrm{b}, \mathrm{z}) \vee\left(([\mathrm{d}])_{0}=1 \& \mathrm{R}_{1}\left(\mathrm{a}, \mathrm{b},([\mathrm{d}])_{10}\right)\right)\right) \supset 1=0$. $4,8$.

From 9 we obtain (ii).

THEOREM 7.1. If A abbreviates the formula $\forall x\left(\forall z>R_{1}(x, x, z) \vee \exists z ~ R_{1}(x\right.$, $\mathrm{x}, \mathrm{z})$ ), then $7 \mathrm{By}\left(\mathrm{y}(\mathrm{A})\right.$ is provable in $S_{3}$.

1. $a @ A \supset \forall x_{a}\left(a(x) \circledast\left(\forall z>R_{1}(x, x, z) \vee \exists z R_{1}(x, x, z)\right)\right)$. D7.

2. $a ® A \supset \forall x \quad \exists y\left(R_{1}(a, x, y) \&[y] \circledast\left(\forall z>R_{1}(x, x, z) \vee \exists z R_{1}(x, x, z)\right)\right.$ ). Unabbreviating 1.

3. $a ® A \supset \forall x$ Gy $\left(R_{1}(a, x, y) \&\left(\left(([y])_{0}=0 \&([y])_{1} @ \forall z>R_{1}(x, x, z)\right)\right.\right.$ $\left.\left.V\left(([y])_{0}=1 \&([y])_{11}=0 \& R_{1}\left(x, x,([y])_{10}\right)\right)\right)\right) .2, D 3, D 1, D 6$.

4. $a @ A \supset \forall x \exists y\left(R_{1}(a, x, y) \&\left(\forall z>R_{1}(x, x, z) \vee\left(([y])_{0}=1 \& R_{1}(x\right.\right.\right.$, $\left.\left.\left.\left.\mathrm{x},([\mathrm{y}])_{10}\right)\right)\right)\right)$. 3; Lemma 32, (i).

5. $a \circledast A \supset \mathcal{A}\left(R_{1}(a, a, y) \&\left(\forall z>R_{1}(a, a, z) \bigvee\left(([y])_{0}=1 \& R_{1}(a, a\right.\right.\right.$, $\left.\left.\left.([y])_{10}\right)\right)\right) .4$.

6. $a ® A \supset 1=0.5$; Lemma 32, (ii).

7. 7 Эy(y®A). 6 .

THEOREM 7.2. If $\mathrm{B}$ abbreviates the formula $\forall \mathrm{x}\left(\forall z>\mathrm{T}_{1}(\mathrm{x}, \mathrm{x}, \mathrm{z}) \vee \mathrm{G} z \mathrm{~T}_{1}(\mathrm{x}\right.$, $\mathrm{x}, \mathrm{z})$ ), then $7 \mathrm{H} \mathrm{y}(\mathrm{y} \AA \mathrm{B})$ is provable in $S_{3}$.

First, we shall show that the formula $\mathrm{BwU}(\mathrm{d}, \mathrm{w})$ is provable in $S_{3}$, where $\mathrm{U}(\mathrm{d}, \mathrm{c})$ is the formula which was defined in $\$ 11$ to represent $U(d)=c$ in $S_{3}$. To begin, we have the provability in some system $\mathfrak{S}$ of the formula $U(d)$ $=\mathrm{c} \equiv \exists \mathrm{Gx}\left(\mathrm{R}_{1}(\mathrm{u}, \mathrm{d}, \mathrm{x}) \&[\mathrm{x}]=\mathrm{c}\right)$ (Formula 2, §11). The formula $\mathrm{U}(\mathrm{d})=\mathrm{U}(\mathrm{d})$ 
is also provable in $\mathfrak{S}_{\text {; thus }} \operatorname{\exists x} \operatorname{\exists y}\left(\mathrm{R}_{1}(u, d, x) \&[\mathrm{x}]=\mathrm{y}\right)$ is provable in $\mathfrak{S}_{\text {. }}$ By the corollary to Lemma 26 , the latter formula will also be provable in $S_{\mathbf{8}}$. Hence, by Formula 5 of $\S 11$, the following formula is provable in $S_{3}$ :

1. $\exists w U(d, w)$.

2. $\exists z T_{1}(a, a, z) \equiv \exists w \exists z\left(T_{1}(a, a, z) \& U(z, w)\right) .1$.

3. $\forall z 7 \mathrm{~T}_{1}(\mathrm{a}, \mathrm{a}, \mathrm{z}) \equiv 7 \mathrm{Hw} \mathrm{Bz}\left(\mathrm{T}_{1}(\mathrm{a}, \mathrm{a}, \mathrm{z}) \& \mathrm{U}(\mathrm{z}, \mathrm{w})\right) .2$.

4. $\exists w \exists z\left(R_{1}\left(\left\{v_{1}, a\right\}, a, z\right) \&[z]=w\right) \equiv \exists z R_{1}\left(\left\{v_{1}, a\right\}, a, z\right)$.

5. $\mathrm{Hz}_{\mathrm{t}}(\mathrm{a}, \mathrm{a}, \mathrm{z}) \equiv \mathrm{Hz} \mathrm{R}_{1}\left(\left\{\mathrm{v}_{1}, \mathrm{a}\right\}, \mathrm{a}, \mathrm{z}\right) .2,4$, Formula $6, \S 11$.

6. $\forall z>T_{1}(a, a, z) \equiv \forall z>R_{1}\left(\left\{v_{1}, a\right\}, a, z\right) .3,4$, Formula $6, \S 11$.

Let $C$ abbreviate $\forall x\left(\forall z>R_{1}\left(\left\{v_{1}, x\right\}, x, z\right) \bigvee \exists z R_{1}\left(\left\{v_{1}, x\right\}, x, z\right)\right)$. Then: 7. $\mathrm{B} \equiv \mathrm{C} .5,6$, definition of $\mathrm{C}$.

Also, by a derivation similar step for step to the proof of Theorem 7.1, replacing $\AA^{\circledR}$ by $\AA_{1}$ and applying Lemma $10,10.7$ and Theorem 4:

8. 7 寻 $y(y \circledast C)$.

9. 7Эу $(\mathrm{\bigotimes} 囚) .7,8$; Corollary 4.2.

Corollary. If $\mathrm{D}$ abbreviates the formula $\forall \mathrm{x} \mathrm{Gy}\left(\left(\mathrm{Bz} \mathrm{T}_{1}(\mathrm{x}, \mathrm{x}, \mathrm{z}) \& \mathrm{y}=0\right)\right.$ $\left.\mathrm{V}\left(\mathrm{Vz}>\mathrm{T}_{1}(\mathrm{x}, \mathrm{x}, \mathrm{z}) \& \mathrm{y}=1\right)\right)$, then $7 \exists \mathrm{y}(\mathrm{y} @ \mathrm{D})$ is provable in $S_{3}$.

The corollary follows from the theorem by application of Corollary 4.2, since the formula $\mathrm{D} \equiv \mathrm{B}$ is provable in $S_{\mathbf{3}}$.

\section{BIBLIOGRAPHY}

1. Paul Bernays, Logical calculus, Mimeographed lecture notes, Institute for Advanced Study, Princeton, 1936.

2. Alonzo Church, $A$ set of postulates for the foundation of logic, Ann. of Math. (2) vol. 33 (1932) pp. 346-366

3. - An unsolvable problem of elementary number theory, Amer. J. Math. vol. 58 (1936) pp. 345-363.

4. Gerhart Gentzen, Untersuchungen ibber das logische Schliessen, Math. Zeit. vol. 39 (1934) pp. $176-210$

5. Kurt Gödel, Über formal unentscheidbare Sätze der Principia Mathematica und verwandter Sysleme I, Monatshefte für Mathematik und Physik vol. 38 (1931) pp. 173-198.

6. - Zur intuitionistischen Arithmetik und Zahlentheorie, Erbegnisse eines mathematischen Kolloquiums, vol. 4 (for 1931-1932, published 1933) pp. 34-38.

7. - On undecidable propositions of formal mathematical systems, Mimeographed lecture notes, Institute for Advanced Study, Princeton, 1934.

8. Arend Heyting, Die formalen Regeln der intuitionistischen Logik, Preuss. Akad. Wiss. Sitzungsber. 1930, pp. 42-56.

9. - Die formalen Regeln der intuitionistischen Mathematik, Ibid. pp. 57-71, 158-169.

10. David Hilbert and Paul Bernays, Grundlagen der Mathematik, Springer, Berlin, vol. 1, 1934, vol. 2, 1939.

11. S. C. Kleene, General recursive functions of natural numbers, Math. Ann. vol. 112 (1936) pp. 727-742. 150-155.

12. - On notation for ordinal numbers, Journal of Symbolic Logic vol. 3 (1938) pp.

13. - Recursive predicates and quantifiers, Trans. Amer. Math. Soc. vol. 53 (1943) pp. 41-73. 
14. - On the forms of the predicates in the theory of constructive ordinals, Amer. J. Math. vol. 66 (1944) pp. 41-58. Errata: p. 43, line 12 read " $(4)^{n}$ instead of " $\left(4^{*}\right)^{n} ;$ p. 54, line 4, read " $a$ " instead of " $e$."

15. - On the interpretation of intuitionistic number theory, Journal of Symbolic Logic vol. 10 (1945) pp. 109-124.

16. R6zsa Péter, Über den Zusammenhang der verschiedenen Begriffe der rekursiven Funktion, Math. Ann. vol. 110 (1934) pp. 612-632.

Amherst College,

AMHERst, Mass. 